Florida International University FIU Digital Commons

6-29-2018

\title{
Supervisory Practices in a Virtual Internship Program: A Multi-Case Study
}

Elizabeth Adadi

Elizabeth Adadi, eadad001@fiu.edu

DOI: $10.25148 /$ etd.FIDC006888

Follow this and additional works at: https://digitalcommons.fiu.edu/etd

Part of the Curriculum and Instruction Commons

\section{Recommended Citation}

Adadi, Elizabeth, "Supervisory Practices in a Virtual Internship Program: A Multi-Case Study" (2018). FIU Electronic Theses and Dissertations. 3748.

https://digitalcommons.fiu.edu/etd/3748 


\section{FLORIDA INTERNATIONAL UNIVERSITY \\ Miami, Florida}

SUPERVISORY PRACTICES IN A VIRTUAL INTERNSHIP PROGRAM:

\section{A MULTI-CASE STUDY}

A dissertation submitted in partial fulfillment of

the requirements for the degree of

DOCTOR OF PHILOSOPHY

in

CURRICULUM AND INSTRUCTION

by

Elizabeth Adadi 
To: Dean Michael R. Heithaus

College of Arts, Sciences and Education

This dissertation, written by Elizabeth Adadi, and entitled Supervisory Practices in a Virtual Internship Program: A Multi-Case Study, having been approved in respect to style and intellectual content, is referred to you for judgment.

We have read this dissertation and recommend that it be approved.

$\begin{array}{r}\hline \text { Eric Dwyer } \\ \hline \text { Melody Whiddon-Willoughby } \\ \hline \text { Sarah Mathews, Major Professor }\end{array}$

Date of Defense: June 29, 2018

The dissertation of Elizabeth Adadi is approved.

Dean Michael R. Heithaus

College of Arts, Sciences and Education

Andrés G. Gil

Vice President for Research and Economic Development and Dean of the University Graduate School

Florida International University, 2018 
(C) Copyright 2018 by Elizabeth Adadi

All rights reserved. 


\section{DEDICATION}

This dissertation is dedicated to God; it is through him that all things are possible. 


\section{ACKNOWLEDGMENTS}

This has been an arduous and insightful journey, and I am very, very grateful for

its end. I would like to thank my many professors for the grueling coursework and constructive feedback that has allowed me to grow as an academic expert in my field of study. Additionally, I want to express my gratitude towards my dissertation committee for their steadfast dedication in helping me complete this journey. Finally, I want to thank my family and friends for continuously believing in me, even at times when I doubted my own abilities to succeed. 


\section{ABSTRACT OF THE DISSERTATION \\ SUPERVISORY PRACTICES IN A VIRTUAL INTERNSHIP PROGRAM: \\ A MULTI-CASE STUDY}

by

Elizabeth Adadi

Florida International University, 2018

Miami, Florida

\section{Professor Sarah Mathews, Major Professor}

This study explored certain leadership methods that eSupervisors were utilizing with eInterns through the theoretical lenses of House's (1971) path-goal theory of leadership and the effectiveness of these methods on learning outcomes. The overarching research question that guided this study was: How do eSupervisors contribute to the learning growth of eInterns? A qualitative multi-case study was conducted on a population of eSupervisors, current eInterns (students), and past eInterns (graduates) that were associated with the Virtual Student Foreign Service (VSFS) program. VSFS is a program aimed at exposing students to working opportunities in the government. The findings from this study imply that there are a number of ways in which eSupervisors can contribute to the learning growth of eInterns. These contributions will vary depending on the eInterns, the environmental characteristics, the leadership approach taken, and the motivational factors involved. The significance in applying this research today falls in line with the booming growth of distance education programs the trend of incorporating technology in the classrooms. 


\section{TABLE OF CONTENTS}

\section{CHAPTER}

\section{PAGE}

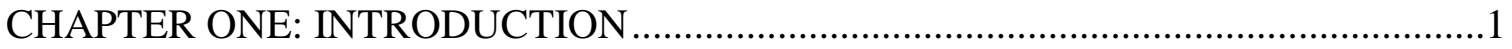

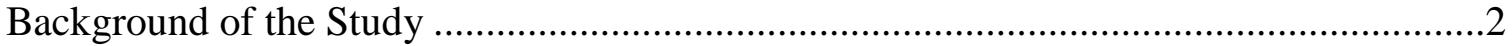

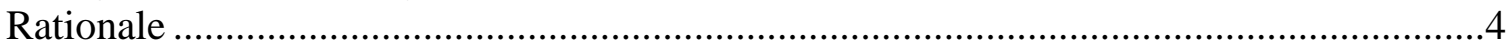

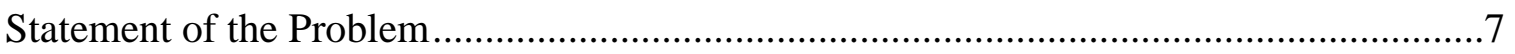

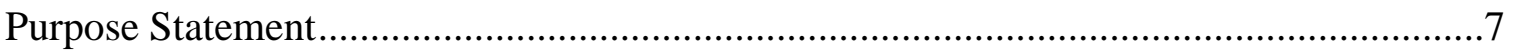

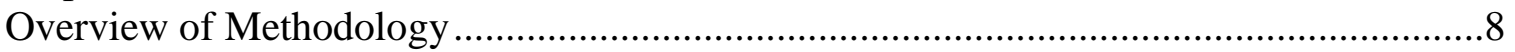

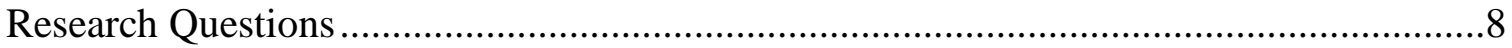

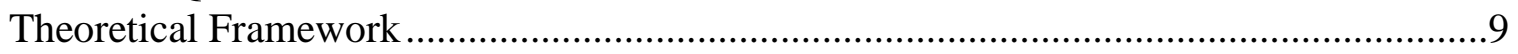

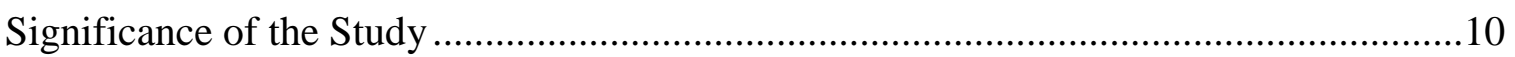

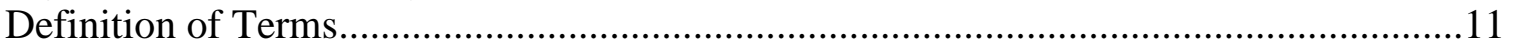

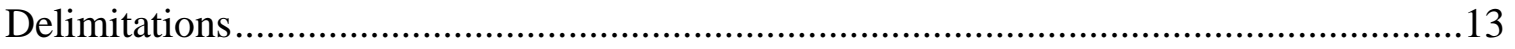

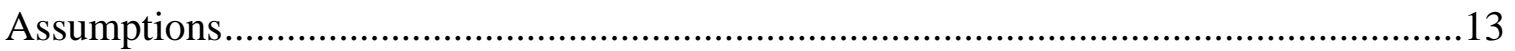

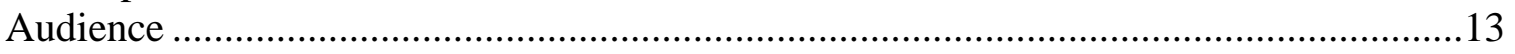

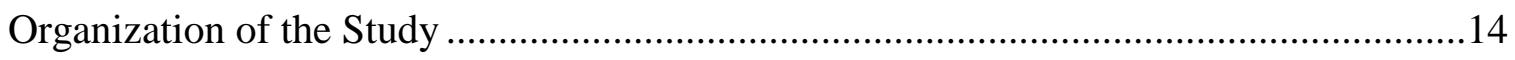

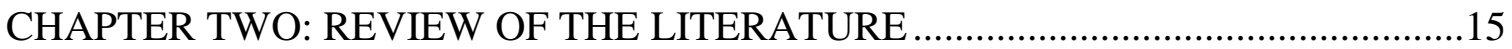

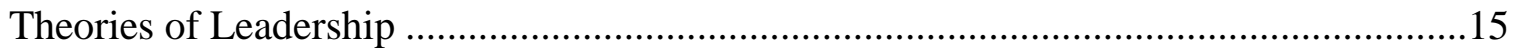

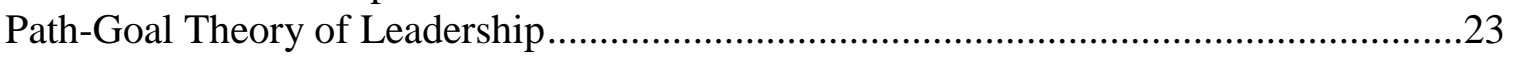

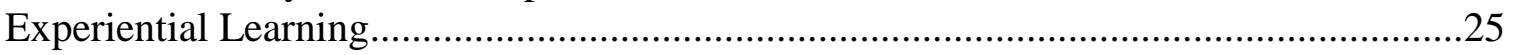

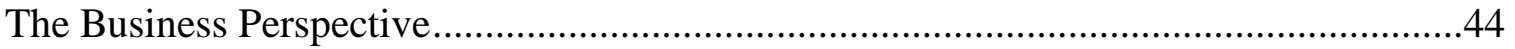

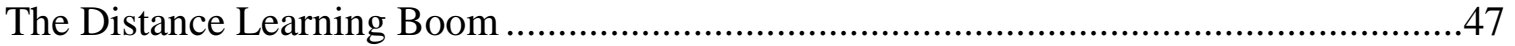

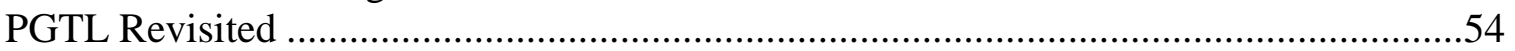

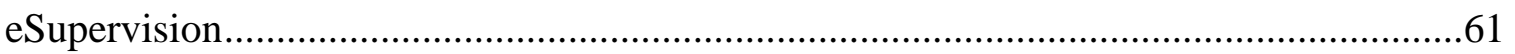

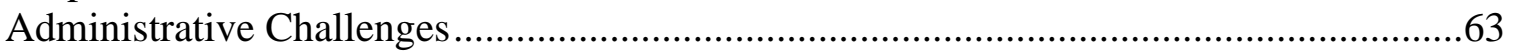

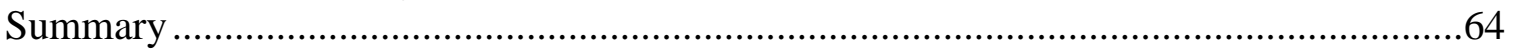

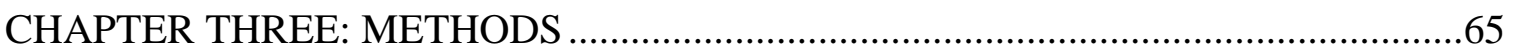

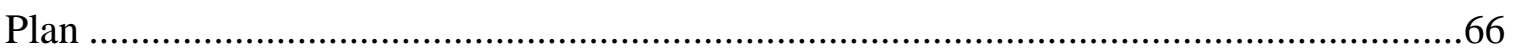

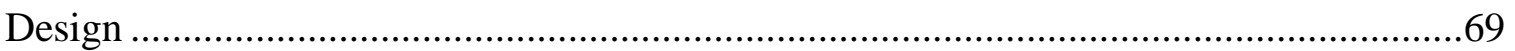

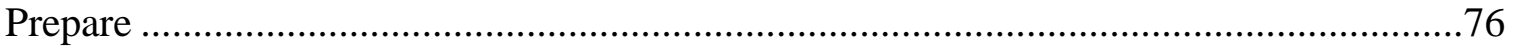

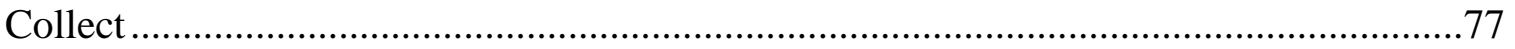

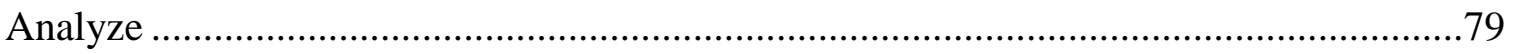

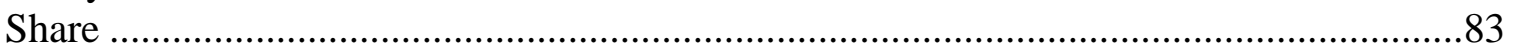

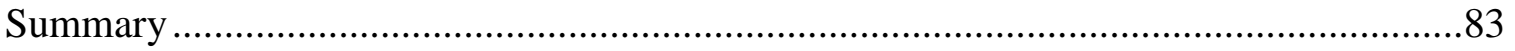

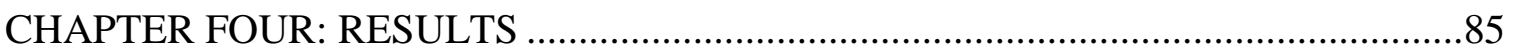

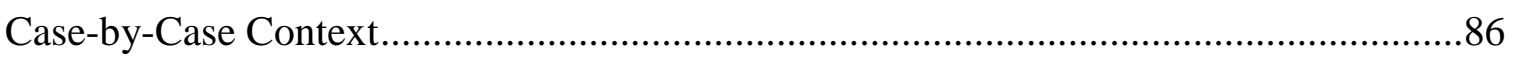

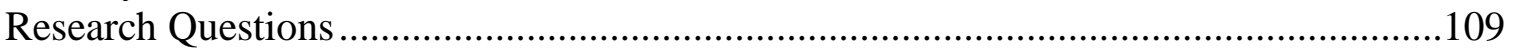

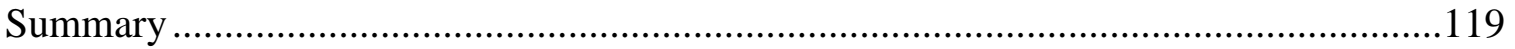




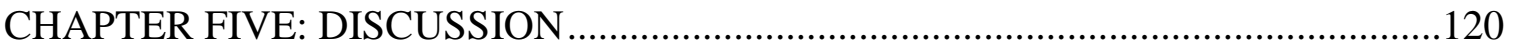

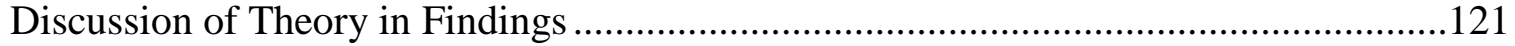

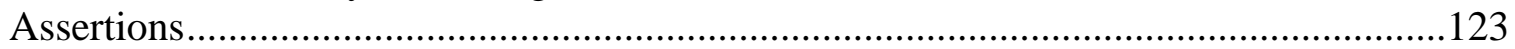

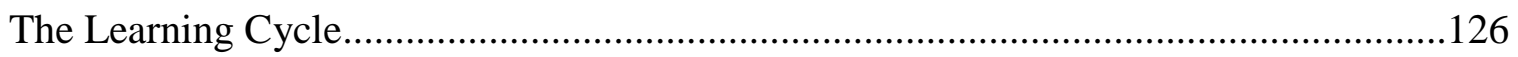

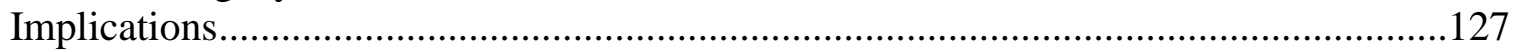

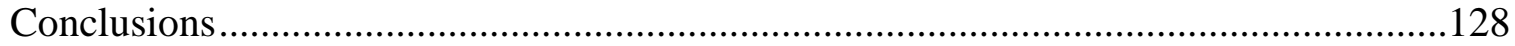

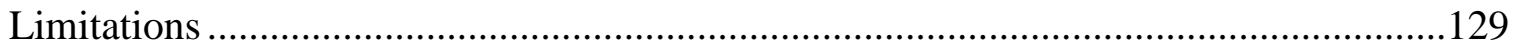

Recommendations for Future Research ................................................................ 129

Recommendations for Practice ............................................................................ 129

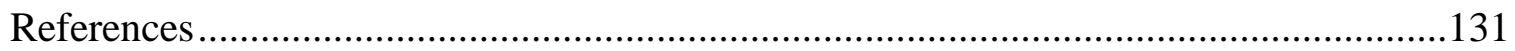

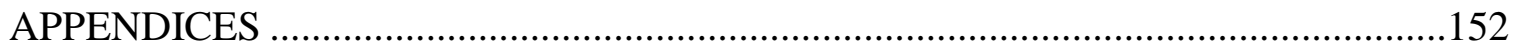

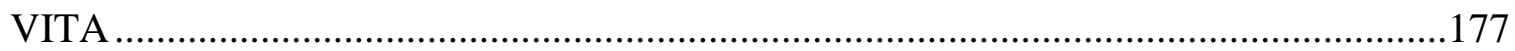




\section{CHAPTER ONE: INTRODUCTION}

Rothman and Sisman (2016) advocate that participating in an internship is a practical way for college students to develop the foundation for a future career. As technologies are increasingly integrated into courses, there is a growing need for the development of strategies that mobilize ways to create collaborative, interactive and relevant practices geared towards student success, specifically within the framework of experiential learning (Howell, Williams \& Lindsay, 2003). Universities can provide an environment that contributes not only to the development of students who are engaged with their studies, but also to the local and global community through experiential learning opportunities. (Acai, Cowan, Doherty, Sharma, \& Thevathasan, 2014).

Internships are experiential in nature because students learn by doing actual work in a career-related field of interest. Internship opportunities are currently offered by $98 \%$ of institutions (Hart Research Associates, 2016). The introduction of technology into experiential learning allows broad access to enable the development of diverse learning communities that may not be possible among geographically bound student populations (Guthrie \& McCracken, 2010). According to Ruggiero (2017), virtual internships have the potential to support the development of cognitive processes and social aspects of professional development.

Virtual workspaces have been made possible through the advances in technology. Virtual interns (eInterns) can work from anywhere, which means that employers can pick from a much larger applicant pool of students who are not tied down to a specific location (Clemmons, 2015). eInterns who are chosen for a job, are usually aligned with a 
supervisor. Merriam-Webster (2017) defines a supervisor as a person who oversees others. In virtual internships, these would be virtual supervisors or eSupervisors. Sweitzer and King (2014) has linked effective supervision to student development and overall learning outcomes.

\section{Background of the Study}

Experiential learning is an approach that helps students relate classroom theory to the world of work (Ruggiero \& Boehm, 2016). This method is considered to be, "the process of creating and transforming experience into knowledge, skills, attitudes, values, emotions, beliefs and senses" (Jarvis, 1987, p. 16). Universities have continuously utilized this "learning through doing" approach as a way of fostering student success (NACE, 2017). There are several different approaches to experiential learning such as undergraduate research, clinical experience, fieldwork, practicums, and internships. Many post-secondary programs include co-op placements, practicums, or internships where students can apply theoretical knowledge to concrete settings. Some institutions also offer courses that incorporate service-learning or community placements (Acai et al., 2014).

Experiential learning helps provide students with the opportunity and motivation to learn new concepts and gain new experiences, which is especially important in preparing them for a professional future where they may change jobs or careers numerous times (Ambrose \& Poklop, 2015). While the focus of experiential learning tends to be on job preparedness, there are many other positive outcomes to be gained from experiential learning, such as opportunities to network, opportunities to experience learning in a different context, and opportunities to grow as an individual (Kolb \& Kolb, 2005). 
Hart, Kremin,and Pasewark (2017) examined an accounting internship program and found that commitment and long-term intentions to work in that particular field might be shaped by early employment experiences with regard to the size of workloads, the scope of work assignments, and the type working relations. While these results cannot be generalized to all fields or general internship programs, the results do point out that the role supervisors play in an internship is pivotal to the intern's success. According to Tourman, Leroux, \& Beney (2012), "supervision of internships [in the] workplace is often based on common sense" (p. 5231). The common notion is that interns will learn by observing experienced professionals and by doing what they see. Any work activity assigned is considered to be productive as well as constructive. With these thought processes in mind, how can one really be sure that the supervisors have the best intent when it comes to supervising their interns? How much do they want their interns to grow or to learn?

One of the most important challenges that exist for internship programs is ensuring that they are educational for students (Stirling, Kerr, MacPherson, Banwell, Bandealy, \& Battaglia, 2017). Many people assume that all experiences are educational although this is not always the case (Moore, 2010). There have been numerous cases identified by the media in which students are exploited through unpaid work experiences or through engagement in meaningless tasks (McKnight, 2013; T. Thompson, personal communication, June 16, 2017). In order for students to reap the benefits of participating in internships, it is essential for internship programs to deliver experiences of high educational quality. An example of such an experience, would be that of a virtual internship. Virtual internships enable those students who have had limited success in 
obtaining experiential learning opportunities, such as online degree-seeking students or students with various obligations, to explore alternative solutions. Jeske and Axtell (2014) even advocate that virtual internships add a certain value to traditional education.

\section{Rationale}

A virtual internship is a form of experiential learning that is used to link the application of theoretical concepts learned in educational settings to a professional practice using telecommunication means only. The emergence of virtual internships suggests that in some disciplines and organizations, where virtual working is established, a virtual internship may be an acceptable alternative to a 'real' experience (Cornelius, Medyckyj-Scott, Forrest, Williams \& Mackaness, 2008). Despite this recognition, there is still resistance in some educational institutions in applying virtual internships at institutions due to the concern about the supervisory processes involved (M. Rosa, personal communication, April 4, 2016). Participating in virtual internship programs would provide an opportunity for non-traditional students, those who have part-time school attendance, work weeks that consists of 35 hours or more, or have dependents, etc. (NCES, 2002; NCES, 2015) to participate in an experiential learning opportunity regardless of their physical location or other responsibilities (Ruggiero \& Boehm, 2016).

Educational outcomes are the desired goals to be achieved by individuals and in turn, society. Although closely linked to educational quality, the outcomes of education are viewed to be integral in meeting society's needs. Research (for example, Feinburg \& Soltis, 2009; Kaufman, Watkins, \& Leigh, 2001) has shown that society requires the standards for success to measure the contributions that educational institutions offer students, future employers, and local communities. As deliberated by Harber (2014), 
"Simply putting more young people into school is not in itself necessarily beneficial to society" (p. 13); determining useful educational results is the first step in achieving measurable educational value-added (Kaufman et al., 2001). Standards of success, however, can be defined differently by multiple parties. For example, an intern's measure of a successful internship can vary significantly from a supervisor's measure. Utilizing one method to determine educational results tends to exclude varying factors or alternate opinions on a measure of success. This study is concerned with observing the actions of the eSupervisors involved in a virtual internship program and exploring the usefulness of their actions towards the virtual interns and society by taking into consideration multiple factors that define success.

A method of observing usefulness is to view the entire process of achieving educational outcomes as a system. A system is a set of interrelated parts, all of which work together towards a defined goal (Dick, Carey, \& Carey, 2015). A system approach to educational planning and achievement would begin with society and includes the school, student, and other educational partners as the beneficiaries of the results achieved (Kaufman et al., 2001). In the case of virtual internships, the goal should be to educate virtual interns on how to assimilate into a working society. Through a system approach, we would be viewing how eSupervisors are working with their eInterns in efforts to meet this goal, how eInterns are working with their eSupervisors, and how these efforts might be able to translate into useful educational results.

The Office of eDiplomacy, a branch of the U.S. Department of State responsible for using technology to make diplomacy efforts more productive, created the Virtual Student Foreign Service (VSFS) program in 2009 (VSFS, 2017). This virtual internship 
program gives students an opportunity to work on projects that advance the work of over 32 federal agencies in the United States government (VSFS, 2017). These projects include economic and political reporting, graphic design, data analysis, application building, and developing virtual programs among others. Interns in this program work about 10 hours a week from September through May. VSFS has been hailed as an innovative program that offers high quality virtual internships along with unique mentoring and exposure to aspiring government workers (ASU Now, 2016; U.S. Mission Haiti, 2017; UVA, 2017).

The literature holds ample evidence about the usage and growth of internship programs; however, it is rather short on evidence that shows what the key components of a successful virtual internship experience entail and how supervisors play a role in this experience as well (Sawyer, 2017). Chapter Two will provide more details on this topic. There is also a lack of research on the use, implementation, and experience of virtual internships and how they may influence student success. Finally, there is also a limited amount of research on how supervisors play a role in the success of virtual interns. The roles of observation, action, and supervision remain partly unknown (Tourman et al., 2012). An internship program has the potential to be transformative, but only if it is designed properly and has positive intentions for the outcomes of the interns. In view of the increased emphasis on online education, student success, and the rise of virtual internships, this study aims to discover learning experiences behind the components of a virtual internship program. 


\section{Statement of the Problem}

The problem to be explored is the lack of research on the effects of eSupervisory relationships with eInterns and their learning contributions to society. How do eSupervisors contribute to the learning growth of eInterns? Research on online graduate students' levels of importance and satisfaction has highlighted career services as an area of focus for potential improvements (Milman, Posey, Pintz, Wright, \& Zhou, 2015). According to the Online Learning Consortium, the growth rate in distance education courses continues to exceed the overall growth rate in higher education (Allen \& Seaman, 2010a, 2010b, 2015). Allen and Seaman (2015) conducted a survey in 2014 which revealed that $70.8 \%$ of postsecondary institutions nationwide agree that distance education is critical to the long-term strategy of their institution. An increase in distance learning students along with preparing students for seamless career integration calls for an enhancement to the career related services offered to students through the usage of information and communications technology.

\section{Purpose Statement}

The purpose of this study is to explore certain leadership methods that eSupervisors are utilizing with eInterns through the theoretical lenses of House's (1971) path-goal theory of leadership and the effectiveness of these methods on learning outcomes. This study also aims to fill in the gap of the lack of research on the effects of eSupervisory relationships with eInterns and their learning contributions to society as well as the gaps about supervisory styles in online learning. 


\section{Overview of Methodology}

A qualitative multi-case study analysis was conducted on an existing virtual internship program to examine the supervisory practices involved in virtual internship environments and how they inform us of certain learning outcomes. According to Stake (2013), multi-case studies are conducted to examine instances with a lot of parts. In this study, for example, although the eSupervisors and eInterns were participating in the same virtual internship program, each participant came from a diverse background, had a different set of goals, and various work requirements that contributed immensely to the quality of the case. The use of a case study approach also allowed the researcher to gain information from the eSupervisors as well as various factors that influenced that eSupervisory style.

\section{Research Questions}

The following research questions will be examined:

1. How do eSupervisors describe their delivery of educational contributions that promote learning in a virtual internship environment?

a. What do eSupervisors want their eInterns to learn during their virtual internships?

b. How are eSupervisors helping eInterns accomplish their learning goals?

2. How do eInterns interpret the educational contributions that they receive from their eSupervisors into learning experiences?

a. What do current eInterns wish to learn during their virtual internships?

b. What do current eInterns perceive they are learning during their virtual internships? 
3. How do virtual internships deliver educational contributions that add value to an eIntern's post-internship experience?

a. How do past eInterns describe what they learned during their virtual internships?

b. How do past eInterns describe how they are applying the skills they have learned from their virtual internship experience?

\section{Theoretical Framework}

The path-goal theory of leadership (PGTL) is a theory specifies a leader's behavior that best fits the employee and the work environment to achieve a goal (House \& Mitchell, 1974). PGTL has become popular in research interested in the effects of this theory in an organizational setting (Bolen, 2015; Haney, 2017; Larson, 2015). According to Yukl (2013), leader influence is usually explained in terms of how the leader causes the subordinate to be more capable of accomplishing tasks. The essence of PGTL is the proposition that "leaders, to be effective, engage in behaviors that complement subordinates' environments and abilities in a manner that compensates for deficiencies and is instrumental to subordinate satisfaction and individual work unit performance" (House, 1996, p. 324). The concept is that the leader adjusts his/her style of behavior to the employee and task characteristics so that the employee can be motivated to excel at their goal (Clark, 2013). Do supervisors in virtual internship programs adjust their behaviors to accommodate for virtual interns? The results of this study present interesting findings. 


\section{Significance of the Study}

The significance in applying this research today falls in line with following the trend of incorporating technology in the classrooms and the booming growth of distance education programs. The digital learning landscape far outmatches the number of opportunities for success that are made available to non-traditional students. Student success is often the result of the teaching, learning, and communication processes that occur during the span of typically any academic course (Cicco, 2014). A crucial aspect of fostering student success is preparing graduates for career integration and bridging the gap between school and society. To illustrate, preparing graduates at all levels for seamless career integration has become one of many strategic priorities at Florida International University (FIU, 2016).

Integrating the use of experiential learning opportunities in education has been shown to prepare students for graduation and assimilation into the workforce (Aldas, Crispo, Johnson, \& Price, 2010; Hart, 2008; Jiusto \& Diabiasio, 2006; Lee \& Dickson, 2010; Retallick \& Steiner, 2009; Simons, et al., 2012). As mentioned previously, experiential learning is an approach that helps students relate classroom theory to the world of work and has been a method for encouraging student success. Experiential learning opportunities can help students explore their interests, test out their skills, and gain valuable work experience while also being exposed to professionals in the field. These learning opportunities, however, have been limited to traditional students who take classes on campus. Students who usually have various obligations that restrict their participation in such activities, find themselves at a disadvantage. Therefore, to be 
inclusive of the non-traditional student population, research on alternative modes of conducting internships is necessary.

Also, the study of the effects of leadership and its importance in the work environment shares many controversies to be explored (Yukl, 2013). According to Yukl (2013), behavioral scientists believe the phenomenon of leadership to be important for the effectiveness of organizations regardless of the various definitions of leadership that have been developed. Whether leadership should be viewed as a specialized role or as a shared influence process is one major controversy of leadership (Antonakis, Cianciolo, \& Sternberg, 2004; Yukl, 2013). Other popularly debated controversies over leadership concern the purpose of influence and the outcomes that these attempts produce and which type of influence is exerted and the outcome of this influence (Yukl, 2013).

In exploring the societal contributions that are being made by the efforts of the supervisors in this virtual internship program, this research will serve as a stepping-stone that will open the door for more research to come. The results generated from this case study will be used to attract attention to the perceived educational outcomes that the system of eSupervisors and eInterns are working towards. Finally, the results that stem from this study will contribute to the literature in the areas concerning leadership methods that eSupervisors are utilizing with eInterns, House's (1971) path-goal theory of leadership, the lack of research on the effects of eSupervisory relationships with eInterns, and experiential learning opportunities for the non-traditional student population.

\section{Definition of Terms}

For this study, the following terms are explained. 
Experiential learning. A broad spectrum of educational experiences, such as community service, fieldwork, sensitivity training groups, workshops, internships, cooperative education involving work in business and industry, and undergraduate participation in faculty research," (McKeachie, 2002, p. 246)

High-Impact Practices. Practices in educational institutions that demand a substantial amount of time and effort, facilitate learning outside of the classroom, require meaningful interactions between faculty and students, encourage collaboration with diverse groups of people, and provide timely feedback (NSSE, 2017).

Internship. Structured and career relevant work experiences obtained by students prior to graduation from an academic program (Taylor, 1988).

Leadership. A supervisor's style and behavior in relation to setting the goals and the agenda for a team (Dansereau, Yammarino, Markham, Alutto, Newman, Dumas, \& Nachman, 1995).

Non-traditional Students. There are many characteristics that categorize a nontraditional student such as, but not limited to, delays in enrollment, part-time attendance, work weeks that consists of 35 hours or more, or has dependents (NCES, 2002; NCES, 2015). In addition, nontraditional students are usually adults ranging from age 25 and up, who are returning to school after a lapse in a few years, and who have other obligations outside of their studies to take care of (Crawley, 2012).

Virtual Internship. An experiential learning activity that allows an intern to work remotely using applications such as the phone and internet (Clemmons, 2015).

Supervisor. A person who oversees others (Merriam-Webster, 2017). 


\section{Delimitations}

Out of the various virtual internship programs in existence, only eInterns and eSupervisors from VSFS participated in this study. The eIntern participants consisted of those who were currently in the internship program as well as those who have graduated from the program. The eSupervisor participants consisted of those who had a supervisory relationship with the eIntern participants.

Another limitation to this study was that demographic characteristics such as race, age, or gender were not considered during the analysis. The cases were selected based on the availability of participants and on the diversity of the demographic combinations. This study was less focused on the demographic relationships and more focused on the learning outcomes due to the virtual context in which the study took place. Demographic characteristics may influence supervisory relationships in a virtual environment, however more research would need to be conducted on this aspect.

\section{Assumptions}

This study assumed that all participants answered the interview questions honestly and to the best of their ability. This study also assumed that eSupervisors have to make certain adjustments to accommodate for their eInterns. Finally, this study assumed that the multi-case design allows for a wealth of knowledge to be gained from the participants in the study.

\footnotetext{
Audience

Beneficiaries of this study will be those personnel involved with, or who have, an interest in the success of students who participate in experiential learning opportunities, especially internships. These personnel include, but are not limited to administrators,
} 
faculty, staff, students, and supervisors involved in virtual internship programs. Research indicates that internships are an important component of higher education programs and serve to build connections between students and future employers (Bohling, Hough, Krinsky, Saleem, \& Stevens, 2012). The overall success of a virtual internship program is strongly dependent on the extent to which a student perceives their experience to reflect a practical situation (Bayerlin, 2015). Supervisors of virtual internship programs would need to exemplify leadership qualities and tactics that would bear the same merit in a face-to-face situation. Findings from this study may be able to give decision makers insight into various factors that are necessary in the implementation of transformative learning experiences in a virtual environment.

\section{Organization of the Study}

The research for this study is organized into five chapters. Chapter 1 introduces the problem, presents the background evidence, and justifies the significance of the study. Chapter 2 presents a review of the literature that encompasses this study. Chapter 3 provides the layout for the study's methodology. Chapter 4 generates the results that stem from the research implementation and Chapter 5 discusses and concludes the study. 


\section{CHAPTER TWO: REVIEW OF THE LITERATURE}

The introduction of virtual internships in experiential learning have opened the amount of learning opportunities available to students (Bayerlin, 2015; Clemmons, 2015; Ruggiero \& Boehm, 2017). Experiential learning opportunities have been shown to have a range of positive effects on student success. The supervisory practices involved in a virtual internship program, however, are still up for debate. The aim of this study was to analyze the roles of eSupervisors when it came to supervising virtual interns.

The literature review first provides background on the leadership theories that were considered for this study, including a highlighting of House's (1971) Path-Goal Theory of Learning (PGTL), which serves as the key construct for this research. Next, experiential learning opportunities and their facilitation of student success are reviewed. The review then highlights internships and their role in student success. The introduction and expansion of distance learning opportunities brings us to narratives regarding the need for virtual internship programs. Finally, the literature review closes with descriptions of effective forms of leadership behaviors that can aid in supervisory processes.

\section{Theories of Leadership}

According to Yukl (2013), understanding leadership as a shared process in an organization or team and how that process delivers desired outcomes has been a growing interest amongst social scientists since the twentieth century. Researchers have considered effective leadership to be a chief element in the success of organizations (Landis, Hill, \& Harvey, 2014). The term leadership has always been defined by researchers according to aspects of the phenomenon of most interest to them (Yukl, 
2013). For example, Lian and Tui (2012) define leadership as the ability to influence others to get things done, while Lunenburg (2012) defines a leader as someone with vision and personal power. Traits, behaviors, influence, role relationships, and interaction patterns are all terms that have been used to describe the concept of leadership at one point or another (Northouse, 2013; Yukl, 2013). Stogdill (1974) pointed out that the number of definitions of leadership can approximate the quantity of people who have tried to define it. Nevertheless, leadership has mostly been defined as involving a process of intentional influence being used over others to structure or facilitate activities and relationships in a group or organization (Yukl, 2013).

An initial review of the literature on leadership revealed a plethora of leadership theories that have developed throughout history (for example, Ahmed, Nawaz, Khan, 2016; Landis et al., 2014; Yukl, 2013). Most of these theories have been categorized according to the traits most likely used to describe or define them. Based on recurring information discovered during the literature review, the theories presented in this research are organized into the following categories: Naturalistic theories, behavioral theories, transactional theories, transformational theories, and contingency theories. To determine which leadership theory best fits the purpose of the study, a thorough examination of the various theories of leadership was performed. Each leadership category identified above will be briefly described in this document, followed by a justification as to why the pathgoal theory of leadership was chosen to frame this study.

\section{Naturalistic Theories}

Naturalistic theories were the first theories of leadership to be developed on the assumptions that leaders were born, not made. Societies used examples of leaders such as 
Alexander the Great, King Arthur, Abraham Lincoln, and Gandhi to hold together this belief (Yukl, 2013). A popular naturalistic theory was great man theory, first introduced by Thomas Carlyle (Carlyle, 1840). It distinguishes an eventful man, one who does not determine the events of history, and an event-making man, one who influences the course of events. Carlyle's inspiration for the theory came from his research and writings on influential heroes. Without any scientific backing, this theory was later challenged as being morally flawed when leaders, such as Hitler and Napoleon emerged (Ahmed et al., 2016) and since women leaders were virtually ignored.

After the incorporation of the scientific method into psychology, researchers assumed that it would be possible to identify certain qualities that differentiated leaders from followers (Kohs \& Irle, 1920). Hence, a new theory of leadership emerged: trait theory. The term trait refers to a variety of personal characteristics such as personality, temperament, motives, and values (Yukl, 2013). Early theorists based this theory on two types of traits that leaders must possess to be effective: emergent traits and selfconfidence and effectiveness traits (Ekvall \& Arvonen, 1991). Emergent traits are dependent upon heredity and include aspects such as height, attractiveness, and intelligence. Self-confidence and effectiveness traits are based on experience or learning. Trait theory focused on analyzing physical, mental, and social characteristics to determine the trait or combination of traits that were common amongst leaders. This task became exceptionally difficult as almost 18,000 personality-relevant terms had been identified by trait theorist Gordon Allport and his associate Henry Odbert (John \& Robins, 1993). This theory lost ground due to its inability to identify which traits 
effective leaders had in common (Ahmed et al., 2016). Allport's studies on personality traits eventually paved the way for behavioral theories.

\section{Behavioral Theories}

As the name suggests, behavioral theories focused on the behaviors of leaders as opposed to their physical, mental, or social characteristics. With the evolution of science, researchers could measure the cause and effects relationships of specific behaviors from leaders (Taylor, 1994; Yukl, 2013). Observed behaviors were classified into various leadership styles and conditioning methods were identified to make leaders instead of waiting for them to be born. In early studies, researchers identified three types of leadership decision-making styles: autocratic, where leaders make decisions alone; democratic, where leaders involve others in the decision-making process; and laissezfaire, where leaders let other make the decisions (Lewin, Lippit, \& White, 1939). Other studies conducted at Ohio State University classified two groups of behaviors that were strongly correlated among common leader behaviors: initiating structure, leaders concerned with tasks; and consideration, leaders concerned with people (Halpin \& Winer, 1957).

\section{Transactional Theories}

Transactional leadership theories focus on the exchanges between leaders and followers (Ahmed et al., 2016). They have been described as that in which leaderfollower associations are based upon a series of agreements between the two (House \& Shamir, 1993). These theories place value on mutually beneficial relationships. The approach assumes that transactional leaders are efficient when they develop a mutual reinforcing environment that aligns the individual and organizational goals. The basic 
idea is that people will be motivated to do things if they will be adequately rewarded for doing so. Leader-member exchange (LMX) is a common theory associated with this approach (Graen \& Uhl-Bien, 1995). According to LMX, exchange relationships are formed gradually through reciprocal reinforcement of certain behaviors as the exchange cycle repeats itself (Yukl, 2013).

\section{Transformational Theories}

Transformational leadership theories assume that leaders can transform their followers through inspirational nature, motivational etiquette, and charismatic personalities (McGregor, 2003). Researchers first became interested in emotional and symbolic aspects of leadership in the 1980's due to the need to understand how followers make self-sacrifices and put the needs of an organization above their own (Yukl, 2013). Transformational leaders are asked to focus on followers' needs and input to transform them into leaders by empowering and motivating them (House \& Aditya, 1997). Burns (1978) transformational leadership theory, which focuses on motivations and values in assessing how a leader approaches power, and Bass (1995) transformational leadership theory, which focuses on how a leader influences followers, are two examples associated with this approach.

\section{Contingency Theories}

Due to the failure of research on leadership to provide strong evidence for universal conceptions of leadership, researchers began developing theories that explained why certain traits or behaviors of leaders were effective in different situations (Yukl, 2013). These theories are categorized as contingency theories. Contingency theories gave rise to the notion that leadership styles should be based on certain situations. These 
theories concentrate on a leader's effectiveness given various situations. A contingent style of leadership is based upon the relationship between the leader and the subordinate. The relationship between the two will determine if the leader is relationship oriented or task oriented.

Many of the early theories associated with this category include Fiedler's (1964) LPC contingency theory, House's (1971) Path-Goal Theory of Learning (PGTL), HerseyBlanchard's (1979) situational leadership theory and cognitive resources theory (Fiedler \& Garcia, 1987). LPC contingency theory and PGTL are two popular contingency theories.

Fiedler (1971) believed that leaders will be effective if they are placed in situations that align with their skills. Fiedler's contingency model uses a least preferred coworker (LPC) score to moderate the effects of group performance on leader traits (Yukl, 2013). He identified three variables that can impact a situation: the relationship between the leader and followers, task structure, and position power. Fiedler's theory lacks flexibility in that it assumes leaders have fixed styles of leadership and cannot change the way a situation is handled.

House's (1971) PGTL proposes that a follower's motivations are based on three assumptions: 1) If effort is given, the goal can be achieved; 2) If the goal is achieved, there will be a reward; and 3) The reward is valuable. There are four styles of leadership associated with PGTL: directive, providing direct and authoritative communication; achievement-oriented, setting high expectations; participative, working with followers; and supportive, showing care and concern. House believed that leaders can change styles and should attempt to change to be effective. Where Fiedler's theory focuses on leader 
influence on group processes (Yukl, 2013), House's PGTL focuses on dyadic influences on subordinates.

Hersey-Blanchard's situational leadership theory (Hersey, Blanchard, \& Natemeyer, 1979) is concerned with the use of a leader's ability to lead in each situation; however, it places followers into groups based on their maturity levels and assigns a leadership style to each group. Maturity levels are determined by task skills and motivation. There are four styles of leadership in this theory: telling, which involves giving commands; selling, which involves providing direction and guidance; participating, in which leaders work equally with followers; and delegating; which involves empowering followers by giving them more responsibility.

Cognitive resource theory (Fiedler \& Garcia, 1987) highlights stress as a factor affecting how people lead. The more intelligent, or cognitively acute, a leader is the more likely he or she will be able to overcome the effects of stress. Cognitive resource theory has a specific model which helps to predict which levels of intelligence are needed for which levels of stressful environments. Issues associated with this theory were that intelligence and stress are not specifically defined and are often measured subjectively.

\section{Controversies}

According to Yukl (2013), the various definitions of leadership have given pause for concern in its use as a scientific construct; however, behavioral scientists believe the phenomenon of leadership to be important for the effectiveness of organizations. Whether leadership should be viewed as a specialized role or as a shared influence process is one major controversy of leadership (Antonakis, Cianciolo, \& Sternberg, 2004; Yukl, 2013). 
Specialized roles place emphasis on the leader, while shared influence places emphasis on the group and the various roles played by each member that influence the group.

Another popularly debated controversy over leadership concerns the purpose of influence and the outcomes that these attempts produce (Yukl, 2013). Some theorists believe that leadership occurs when people are influenced to do ethical or beneficial acts (Mathooko, 2013; Mayer, Aquino, Greenbaum, \& Kuenzi, 2012). Other theorists believe that leadership includes all attempts to influence attitudes and behaviors of followers (Kellerman, 2007; Lian \& Tui, 2012).

Yukl (2013) acknowledges a third controversy of leadership involving which type of influence is exerted and the outcome of this influence. For example, influencing subordinates through empowerment or through coercive techniques (Lunenberg, 2012) is one such controversy. The difference between influence that results in enthusiastic commitment by followers versus reluctant obedience or indifferent compliance has been contested among some theorists (Carson, Carson, \& Roe, 1993; Tracy, 1990). Nonetheless, it has been acknowledged that using different types of influence in varying combinations to adhere to the situation at hand has been proven to be effective by the most powerful leaders (Lunenberg, 2012).

Another controversy among leadership components is the role that followers play in influencing leaders (Burak \& Bashshur, 2013; McCallum, 2013). This form of influence is termed followership and involves reversing the focus on followers to balance our understanding of the effects of leadership (Shamir, 2007). This component of leadership moves away from the dyadic lens and focuses on the influence of subordinates as a group on their leader's actions. 


\section{Path-Goal Theory of Leadership (PGTL)}

While there are many more theories and styles of leadership other than those mentioned here, these categories provide an overview of what has been evolving in leadership studies over the years. Naturalistic theories highlight leaders who are born, behavioral theories focus on making leaders by conditioning their behaviors, transactional theories concern themselves with mutually beneficial relationships between leaders and followers, transformational theories aim for leaders to motivate and inspire, and contingency theories are situation-bound. Concerning the parameters of this study, students and supervisors working in a virtual internship program, a situation-bound approach to leadership was necessary. Contingency theories focus on the relationship between the superior and subordinate and aim to explain why certain leadership traits are effective in any given situation. In the context of this study, the eInterns are in a virtual working environment where face-to-face interactions are non-existent. Given this context, leadership theories categorized as contingency theories were further considered.

According to Naylor (1999), a contingency factor is any condition in any applicable environment to be considered when designing elements of an organization. Contingency theories consider factors relating to an environment, which, in turn, would determine the leadership style that can be best applied to the work situation. Aside from leadership style, success with this approach would also depend upon the qualities of followers and situational features (Cherry, 2012).

In comparing the contingency theories previously mentioned, House's (1971)

PGTL incorporates many aspects of situational variables into its concept, while HersheyBlanchard's (1979) situational leadership theory only takes into consideration 
subordinate maturity, Fiedler's (1971) LPC contingency theory only measures task structure in leadership relations, and cognitive resources theory only focuses on stress factors and intelligence levels (Yukl, 2013). Fiedler's contingency theory places an emphasis on the leader and the situations which are most effective for them to lead in while House's theory emphasizes the reactions of the followers in response to the leader's actions. Both PTGL and situational leadership theory associate with specific leader behaviors, however, the LPC contingency theory has none (Yukl, 2013). Situational leadership theory is flexible to the leader, however, it is difficult for supervisors to properly assess the maturity levels of their eInterns given the limited interactions in a virtual environment.

PGTL specifies a leader's behavior that best fits the employee and the work environment to achieve a goal (House \& Mitchell, 1974). This theory also proposes that a follower's motivations are based on certain assumptions. With House's (1971) path-goal theory, the best leadership approach can be identified based on followers' needs, the tasks they are assigned, and the environment that they are working in.

Alignment with motivation theories. Aside from its contingent approach to leadership, PGTL includes a concept of motivation that is based on Vroom's (1964) expectancy theory of motivation. Expectancy theory of motivation proposes the desirability of an outcome to be the motivating factor in behavior selection. According to this theory, an individual's motivation to put forth a certain amount of effort into completing a task is determined by the individual's evaluation of their situation (Porter \& Lawler, 1968; Vroom 1964). 
An individual's evaluation of their work environment, needs, and tasks assigned would result in an estimation of satisfaction with their job. Lawler and Porter (1967) contend that the importance of job satisfaction rests on strong correlations between absenteeism and satisfaction, along with turnover and satisfaction. PGTL surmises that an individual will be motivated to work in an environment where his or her needs will be satisfied, thus resulting in lower absenteeism and turnover rates. According to PTGL, it is a leader's responsibility to select behaviors that will increase a follower's motivation to complete a task (House, 1971). Vroom (1964) cites expectancy, instrumentality, and valence as motivational concepts playing a huge role analyzing and explaining behavior in work environments.

Among the various leadership theories, PGTL is consistent with the expectancy theory in that leaders can impact the task-oriented actions of subordinates by influencing their perceptions about the outcomes stemming from task completion (Yukl, 2013). However, Vroom's theory does not specify which tactics motivate subordinates (Lunenburg, 2011), while PGTL suggests certain conditions must be met to motivate employees.

\section{Experiential Learning}

Experiential learning theory emphasizes experience as playing a central role in the learning process. The emergence of this theory gave rise to a new set of ways in which we can manage education, relationships among learning, work, other life activities, and the creation of knowledge itself (Kolb, 1984). The notion of experiential learning was first distinguished by John Dewey (1916) in the early 1900's. Dewey (1916) highlighted indirect education as being a personal and vital way of transmitting knowledge from 
adults to their young; he cautioned against formal instruction, in its effort to promote the subject matters of the school, as being a method of isolating the subject matters of lifeexperience. "The student learns by doing: or to put this in other words, he tests hypotheses in the laboratory of real life" (Dewey, 1971, p. 10); with this is mind, consider the steps of the scientific method. First, a student would make observations about potential careers in their field of study. Then, that student would generate a hypothesis concerning a career of interest. Next, the student would test out that hypothesis through some sort of experiential learning experience. After gathering data from the experience and analyzing it through a reflective process, the student can decide on whether to test out a new hypothesis or to continuing refining the original one. Dewey (1916) proposed that learning should emphasize the interrelatedness of all things. This perspective on learning was able to emerge through Dewey's work, along with that of other scholars (Kolb, 1984).

\section{Emergence of the Theory}

Beginning with Lewin (1951) in his model of action research and laboratory training, learning, change and growth were thought to best facilitated by a process cycled itself in four stages. First, it begins with the "here and now" experience that is then followed by a collection of data and observations about that experience. Next, data are analyzed and reflected on. The final stage involves determining if behavior needs to be modified for a new set of experiences. Primarily based on feedback processes, this model is remarkably like the scientific method. Lewin believed that an individual's ineffectiveness in learning tasks was due to a lack of adequate feedback processes (Kolb, 1984). 
Dewey (1938) believed in a learning process that was like Lewin's, although, he placed more emphasis on the developmental nature of learning and the formation of purposes. Starting with observations of surrounding conditions, then recalling similar experiences that have happened in the past, followed by putting these observations together and forming judgements, which in turn gives meaning and purpose to any actions that follow. From Dewey's perspective, learning is a dialectic process that integrates experience, concepts, observations, and action (Kolb, 1984).

Piaget's (1970) model of learning and cognitive development considered experience, concept, reflection, and action to form the development of adult thought. He maintained that from infancy to adulthood, development moved from a concrete phenomenal view of the world to an abstract constructionist view, and from an active egocentric view to a reflective internalized mode of knowing (Kolb, 1984). Like Lewin and Dewey, Piaget's view of the learning process involved a cycle of interaction between an individual and the environment. For Piaget, learning was termed intelligent adaptation (Kolb, 1984).

Since early explanations of the experiential learning experience, there have been numerous adaptations to the definition. For example, Kolb (1984) defines experiential learning as "the process whereby knowledge is created through the transformation of experience" (p. 38). On another note, Cantor (1995) defines experiential learning as "active learning," wherein the learner takes responsibility in the learning process. Experiential learning has also been defined as "a broad spectrum of educational experiences, such as community service, fieldwork, sensitivity training groups, workshops, internships, cooperative education involving work in business and industry, 
and undergraduate participation in faculty research," (McKeachie, 2002, p. 246). For the purposes of this research, McKeachie's (2002) definition of experiential learning will be applied as the definition explicitly encompasses this study's topic, research group, and research scope.

Kolb (1984) drew on the work of scholars such as John Dewey, Jean Piaget, William James, and Kurt Lewin to develop an experiential learning theory. These scholars provided insight into the meaning of experiential learning, which were used to develop six propositions that the theory is built on (Kolb and Kolb, 2005):

1. Learning is best conceived as a process, not in terms of outcomes.

2. All learning is relearning.

3. Learning requires the resolution of conflicts between dialectically opposed modes of adaptation to the world.

4. Learning is a holistic process of adaptation to the world.

5. Learning results from synergetic transactions between the person and the environment.

6. Learning is the process of creating knowledge .

\section{Foundations of Experiential Learning}

\section{Learning is best conceived as a process, not in terms of outcomes.}

Traditionally, idealist approaches of education and behavioral theories of learning have been based on the idea that there are elements of consciousness that always remain the same (Kolb, 1984). Through various combinations and associations, these fixed elements form our patterns of thought. This idea of fixed elements of thought has resulted in the tendency of learning to be defined in terms of its outcomes, such as behavioral outcomes 
or the accumulation of knowledge represented by a storehouse of facts (Kolb, 1984). The notion is that it is possible to measure how much someone has learned by the amount of fixed ideas that person has accumulated. Experiential learning theory, however, does not assume that ideas are fixed and immutable. Experiential learning assumes that ideas are formed and reformed through experience. Engaging students in a process that involves feedback on the effectiveness of their learning efforts should be a primary focus in higher education learning (Kolb \& Kolb, 2005).

Learning is a continuous process grounded in experience. Dewey (1938) believed that the continuity of experience was a life process that was central to the theory of learning. Consciousness is continuous (James, 1890); knowledge is derived from and tested out in the experiences of learners (Kolb, 1984); therefore, learning is continuous. The facilitation of learning through a process that enables the student to examine their own beliefs and ideas about a topic and then integrate them with new ideas is thought to be most effective means of educating (Kolb \& Kolb, 2005).

\section{The process of learning requires the resolution of conflicts between}

dialectically opposed modes of adaption to the world. In each model of learning proposed by Lewin, Dewey, and Piaget, it is suggested that learning results from a resolution of conflicts between opposing ways of dealing with the world (Kolb, 1984). Essentially, the learning process is driven by conflict, differences, and disagreement (Kolb \& Kolb, 2005). Lewin (1951) emphasized the conflict between concrete experience and abstract concepts along with the conflict between observation and action. Dewey (1938) highlighted the conflict between the impulse and purpose. The accommodation of ideas to the external world and assimilation of experience into conceptual structures were 
recognized in Piaget's (1970) framework. These models suggest that learning is a conflict-filled process that requires four different kinds of abilities for learners to be effective: concrete experience abilities, reflective observation abilities, abstract conceptualization abilities, and active experimentation (Kolb, 1984). Learners must be able to involve themselves in new experiences fully, openly, and without bias. They must be able to observe and reflect on experiences. They must be able to create concepts and theories from their experiences, and they must also be able to act on these theories to make decisions or solve problems. Successful use of these abilities requires integrating the four adaptive modes in a fluid process to develop creativity and growth.

Learning is a holistic process of adaptation to the world. Learning is not just the result of cognition (Kolb \& Kolb, 2005). Learning involves thinking, feeling, perceiving, and behaving; this is a major process of human adaptation (Kolb, 1984). Experiential learning theory is concerned with how the nature of human functions are integrated by an individual into a holistic adaptive position toward the world (Kolb, 1984). Lewin, Dewey and Piaget all saw the scientific method as the basic processes of human adaptation and as a means for describing the holistic integration of all human functions (Kolb, 1984).

\section{Learning involves transactions between the person and the environment.}

A transaction involves a fluid relationship between objective conditions and subjective experiences (Kolb, 1984). Learning is seen to occur through a balance process of assimilating new experiences into existing concepts and accommodating existing concepts into new experiences (Kolb \& Kolb, 2005). Unlike traditional educational processes, experiential learning contextualizes the relationship between the person and 
the environment. This "environment" involves any conditions needed to for an individual to interact and create experiences with (Dewey, 1938).

Learning is the process of creating knowledge. Understanding the nature and forms of human knowledge is necessary in understanding learning (Kolb, 1984). Experiential learning theory advocates the notion of social knowledge being created and recreated in the personal knowledge of the learner; this is considered to align with a constructivist view of learning (Kolb \& Kolb, 2005). The process of learning entails the transaction between subjective and objective experiences, which results in the creation of knowledge (Kolb, 1984).

\section{The Facilitation of Learning}

Kolb and Fry (1975) proposed a four-stage learning cycle that encompasses the abilities needed for learners to be effective: concrete experience, reflective observation, abstract conceptualization, and active experimentation. The learning process contains two primary dimensions (Kolb, 1984): 1) concrete experience and abstract conceptualization; and 2) active experimentation and reflective observation. In the process of learning, the learner must choose which set of learning abilities to use in any given situation; this will determine the level of learning that results (Kolb, 1984). Kolb and Fry (1975) maintain that the learning cycle is a continuous process that can begin at any one of the four stages, but must follow-through the entire sequence to be effective:

1. Concrete experience. The key to learning in this stage is active involvement. To promote active involvement in an internship environment, a supervisor can have the intern work in a test environment first, before moving onto an actual production environment. 
2. Reflective observation. This stage involves reviewing what has been done or experienced. The supervisor can either discuss a learning experience with the intern, or the intern can keep a reflective journal.

3. Abstract conceptualization. Interpreting events and making sense of what has happened takes place in this stage. The intern can formulate his or her own hypotheses about an experience by comparing what they have done to what they already know.

4. Active experimentation. Finding relevance in an experience and putting what has been learned into practice and concerns this stage. If an intern is having trouble placing experiences into a context that is relevant to them, then the learning experience will lack in effectiveness.

An important step of experiential learning is the translation of new conceptual understanding into "actionable" knowledge that can be applied to and used towards new experiences (Kolb, 1984). For example, experiential learning can help students move beyond repetition of facts and theories and instead learn about complex issues that have local or global relevance (Acai et al., 2014). According to Kolb and Kolb (2005), "learning is best facilitated by a process that draws out the students' beliefs and ideas about a topic so that they can be examined, tested, and integrated with new, more refined ideas" (p. 205). Think back to the scientific method, mentioned earlier in this chapter. Experiential learning is a necessary form of learning that helps students in fine-tuning their life goals and career paths. Experiential learners have found that this sort of experience has helped to develop their critical thinking skills and has also kept them more 
engaged with their studies, allowing them to better understand the significance of their post-secondary education (Acai et al., 2014).

It is important to note that experience alone does not produce learning. Dewey (1944) states that learning requires the "reconstruction or reorganization of experience that adds to the meaning of that experience and which increases [the] ability to direct the course of subsequent experience" (p. 74). This notation places a direct emphasis on the importance of reflections in experiential learning experiences. The process of critical reflection involves analyzing the assumptions on which beliefs have been built and evaluating the validity of one's understanding of a subject in each setting (Meizrow, 1990). Students should engage in critical reflections before, during, and especially after their experiential learning experience is over (Acai et al., 2014). Whether reflections occur naturally through one's own cognitive processing or through a requirement, they can facilitate the processing of a deeper meaning from the experience (Rothman, 2016).

Criticisms. The question of whether experiential learning programs enhance student learning has been raised numerous times (Austin \& Rust, 2015). Katula and Threnhauser (1999) found that stand alone cooperative education experiences do not enhance student learning; instead, they found that they must be effectively integrated with the academic discipline. Are supervisors in virtual internship programs effectively integrating learning outcomes with academic disciplines?

Krohn (1986) pointed out some of the negative aspects of internship programs, as well, ranging from students not taking their internship seriously to supervisors wasting time advising interns. Would virtual interns take their internships more seriously if supervisors had a more active and influential role in the programs? It has also been 
maintained that internship program administrators are underappreciated and not adequately compensated for their efforts (English \& Lewison, 1979). Does acknowledgement from superiors influence the way supervisors supervise their virtual interns?

\section{The Impact on Student Learning}

Inspired by Dewey's notion of the interconnectedness of things, schools have gradually adopted new approaches to education, such as learning by doing (Westera, 2011). It is said that actual practice in work settings and authentic feedback from employers helps to enhance student learning (Ambrose \& Poklop, 2015). Many practices in education are held with the expectation of learning outcomes that result in student success. There are certain opportunities for undergraduate students that are designated as "high-impact" due to their positive associations with student learning and retention. High-Impact practices have several characteristics in common: They demand a substantial amount of time and effort, facilitate learning outside of the classroom, require meaningful interactions between faculty and students, encourage collaboration with diverse groups of people, and provide timely feedback (NSSE, 2017). The use of experiential learning as a "high impact practice" in education resulted from investigations into the correlation between certain experiences and powerful learning outcomes (Ambrose \& Poklop, 2015; Kuh, 2008). It is thought that learning through direct experiences is more meaningful learning inside of a classroom (Acai et al., 2014).

A study of 716 undergraduate students in the UK found that students who had participated in work integrated learning (interns, practicum, clinicals, etc.) reported significantly higher confidence levels in goal setting and goal attainment (Purdie, Ward, 
Mcadie, King, \& Drysdale, 2013). It has been suggested that this may enhance the student's ability to establish and achieve goals in the workplace. In another example, Simons, Fehr, Blank, Connell, Georganas, Fernandez, \& Peterson (2012) conducted a study of learning outcomes of students enrolled in a practicum/intern program; the responses from field supervisors, students, and faculty revealed that the experience was beneficial to all involved and that the program helped the students acquire an in-depth understanding of the academic content.

Other student outcomes often associated with experiential learning include increased student readiness for self-directed learning; self-confidence; personal, civic, and professional development; increased working relationships; collaboration among faculty and students; and experiences that help students gain employment such as professional networking contacts (Aldas, Crispo, Johnson, \& Price, 2010; Hart, 2008;

Jiusto \& Diabiasio, 2006; Lee \& Dickson, 2010; Retallick \& Steiner, 2009; Simons, et al., 2012).

\section{Situational Applications}

How do students know when to apply the teachings from the classroom into certain situations? To develop the knowledge of "knowing when," students must be able to identify what skills are appropriate to use in the situations they find themselves in (Ambrose, Bridges, DiPietro, Lovett, \& Norman, 2010; Anderson, 1995). By exploring these situations through experiential learning contexts, students will have a broader knowledge base of how to appropriately apply certain skills in work settings. The use of knowledge and skills in new settings will aid in the deepening of their learning (Ambrose \& Poklop, 2015). 
Through research conducted at Northwestern University (Ambrose \& Poklop, 2015), a model for conducting experiential learning programs was developed to enhance a student's knowledge of situational applications:

1. Apply knowledge and skills

2. Gain new knowledge and skills

3. Integrate and use

4. Reflect.

Research shows that experiential learning helps students understand how to apply theory as well as improve their reasoning skills (D'Amato \& Krasney, 2011; Coker, 2010; Victor, 2013). For example, Victor (2013) examined the long-term impact of an outdoor experiential course in literature. Through this qualitative study, four themes were developed from participant interviews. It was found that the course "nurtured creativity; increased collaboration skills; developed self-confidence/self-knowledge; and reinforced the importance of having a relationship with the outdoors" (p. 93). These themes are supported by another qualitative study conducted with participants in an Outdoor Adventure Education course (D’Amato \& Krasney, 2011).

Another example is from research conducted on occupational therapy students. Coker (2010) conducted pre- and post-tests of occupational therapy students who completed a hands-on therapy program. She found that increases in the students' post-test scores on the Self-Assessment of Clinical Reflection and Reasoning and California Critical Thinking Skills Test were statistically significant $(\mathrm{p}<.05)$ after completing the program. 


\section{Types of Experiential Learning Activities}

Experiential learning is defined as "hands-on" learning and may involve any of the following activities: service learning, study abroad, undergraduate research, cooperative education, internships, student leadership, volunteerism and experimental activities (Austin \& Rust, 2015; Coker, Heiser, Taylor, \& Book 2017; Guthrie \& Jones, 2012; McKeachie, 2002). However, in addition to the different definitions of experiential learning, the activities involved are not always clearly distinguished from each other, such as cooperative education, internships, practica, service learning, and volunteerism. To differentiate between some of the activities involved in experiential learning, below is a description of each.

Cooperative education. Cooperative education or co-ops are more structured and combine academic education with practical work experience (Weible, 2009). Cooperative education is a form of employment that supplements the curriculum by enabling students to gain new knowledge and skills not included in coursework (Ambrose \& Poklop, 2015). Co-op placements can be paid or unpaid and the requirements for participation are determined by a student's respective college or degree program. Students who participate in co-ops stop taking classes to work full time from anywhere between three and 12 months. As a result, students are out of class and working for at least a full school year over the course of their college career (Boyington, 2015). For example, Northeastern University hosts a co-op model that requires students to engage in at least six months of full-time work. The model also requires students to work in multiple placements, enabling employers to give them a high level of responsibility and leading to more 
development of their professional skills and hands-on experience (Ambrose \& Poklop, 2015).

Internships. Internship experiences are a common form of experiential learning. Internships are more flexible than co-ops in that students can work part-time and still take classes. During an internship, students learn to make connections between what they are learning in courses and their on-the-job experience. These experiences can also be paid or unpaid. Also, some degree programs may not require participation in an internship to graduate, so involvement is entirely up to the student. In some cases, however, students can receive academic credit for participation in internships. Steffes (2004) suggests that participation in an internship can help students explore particular settings and/or career paths. Researchers have also discussed the idea that students who complete internships have found professional benefits after college such as greater job satisfaction (Austin \& Rust, 2015).

Practica. A practicum is a course of study that involves the supervised practical application of previously studied theories (Mount Holyoke, 2017). The similarity between practica and internships is that they are both supervised discipline-related and career-related work experiences that involve active learning, critical reflection, and professional development (Gavigan, 2010; O’Neil, 2010). However, the scope of a practicum differs in that it is a field experience that allows a student to observe and document how working professionals perform their job responsibilities (Best Counseling, 2017). "Hands-on" experience during a practicum is very minimal and must always involve the supervision of a faculty member or on-site supervisor. Students must also enroll in a course to take on a practicum. 
Service learning. Another common form of experiential education is service learning. According to the National Survey of Student Engagement, these are courses that include community-based projects (NSSE, 2017). An intended outcome of this form of learning is developing a respect for diversity (Cantor, 1995). Researchers (Baldwin, Buchanan, \& Rudisill, 2007) studied the impact that a service learning program had on teacher candidates' respect for diversity and found that this type of learning had a positive influence on their willingness to teach in diverse school settings. The service learning program was even able to expand their perceptions of society as the teacher candidates "even began to question societal inequities that they encountered," (Baldwin et al., 2007, p. 326). There have also been studies that report service learning to positively impact freshman students' esteem and motivation to volunteer for professional growth and develop work-based competencies and global citizenship ideals (Eppler, Ironsmith, Dingle, \& Errickson, 2011; Ramson, 2014).

Volunteerism. A volunteer is an individual who provides service to others with no tangible reward or benefit (Acai et al., 2014). Volunteer opportunities exist both within post-secondary institutions and in the broader community. Given the collaboration between university and community institutions in experiential education and the diverse motivations for encouraging student community involvement, this academic practice can be viewed as a form of student learning and as a service delivery tool for community organizations (Littlepage, Gazley, \& Bennett, 2012).

This study focuses on the virtual modality of internships being that they are the most common form of experiential learning activities, have direct benefits to careerrelated interests, are flexible, and can provide academic credit. 


\section{Internships}

Internship programs were first developed in 1906 at the University of Cincinnati (Weible, 2009). Internships are defined as "structured and career relevant work experiences obtained by students prior to graduation from an academic program" (Taylor, 1988, p. 393). They come in the form of part-time or full-time field experiences in organizational settings that involve a wide variety of academic disciplines (Gault, Redington, \& Schlager, 2000). They are generally conducted by collegiate students prior to graduation and have some relevance to their chosen career paths (Taylor, 1988).

The Association of American Colleges and Universities designated participation in internships as one of 10 "high-impact" practices that foster engagement, persistence, and learning amongst undergraduate students (Brownell \& Swaner, 2010; Kuh, 2008). Considering the overarching positive impact of internships, higher education and student affairs professionals should facilitate pathways for students to engage in internships.

Influential learning theorist, Kolb (1984) posited that knowledge is created by the transformation of experience. As such, the transformation a student's experience may be facilitated by opportunities to reflect on the impact of an internship on occupational choices. The positive connection between an internship and enhanced vocational selfconcept has been reported as further evidence that reflecting on work experience better prepares students for the career industry (Drewery, Nevison, \& Pretti, 2016).

Stichman and Farkas (2005) conducted a nationwide study on internships in undergraduate criminal justice programs and found the major strength of an internship to be the student's' ability to integrate theory and practice. Similarly, Gavigan (2010) reported that the major benefit from a summer internship is the transformation of student 
knowledge. Thus, as acknowledged by Kolb (1984) and Jarvis (1987), an internship can provide students with a transformative experience.

This is the general thought process behind engaging in an internship; First, by having the internship experience, students clarify their possible career paths before they embark on their job search after graduation (Moghaddam, 2011). Second, students gain a realistic view of what the employer expects of them as well as what they can expect from the workplace (Hiltebeitel, Lauby, \& Larkin, 2000). Third, it helps students reflect on personal fit and if they believe they can succeed within a particular job or industry (Lord, Sumrall, \& Sambandam, 2011). "Consequently, internships offer a stepping stone into the world of work whereby students check out industries for future placements, work functions for future jobs and self-concept congruency for person-career fit" (Rothman \& Sisman, 2016, p. 1004)

The number of internship opportunities that are made available to students is of extreme importance. Participation in an internship has been considered such an important part of an undergraduate student's educational experience that several scholars have suggested it be a mandatory requirement (Hiltebeitel et al., 2000; McCarthy \& McCarthy, 2006; Divine, Linrud, Miller, \& Wilson, 2007; Templeton, Updyke, \& Bennet, 2012). Some universities have already begun this transition. For example, Florida International University drafted a strategic plan that discusses the expansion experiential learning opportunities for students as one of its key measurable goals (FIU, 2016).

Impact. Research demonstrates the positive impact that internship experiences have on job offers, compensation, and job satisfaction (Austin \& Rust, 2015; Gault et al., 2000; Hart et al., 2017). For example, a clear majority of accounting interns receive and 
accept full-time offers at the completion of their internship (Hart et al., 2017). The company Intern Bridge found that, across 6,147 students, the level of student satisfaction with an internship was directly correlated with length, ranging from short experiences of 1 to 4 weeks to long experiences of 13 to 16 weeks (Grasgreen, 2012). Pre-teachers in yearlong internships reported greater outcomes than those who participated in a semester internship. These outcomes included better relationships with supervising teachers, greater knowledge of school policies and procedures, and higher scores for the perceived adequacy of time spent in school (Spooner, Flowers, Lambert, and Algozzine, 2008).

Kilgo, Sheets, and Pascarella (2015) conducted a study that tested the impact of high-impact practices on liberal arts educational outcomes and found that participation in internships was positively associated with several college outcomes, such as increased need for cognition, intercultural effectiveness, and socially responsible leadership. In another study that evaluated the difference between college students who participated in internships and those who did not, it was found that college students who participated in internships demonstrated significantly higher fourth-year grades than their counterparts (Parker III, Kilgo, Sheets, \& Pascarella, 2016). This advantage persisted even in the presence of potential confounding influences (i.e., demographic characteristics, academic preparation, previous grades, institutional characteristics, and other college experiences). This finding suggests that the positive benefits of participating in internships may be most beneficial for those starting out in college with the lowest levels of academic achievement (Parker et al., 2016).

Studies also demonstrate that participation in internships has been associated with the enhanced interpersonal, multicultural, and time management skills of students 
(Fernald \& Goldstein, 2013; Jones, 2002; Knouse, Tanner, \& Harris, 1999; Miller, Rycek, \& Fritson, 2011; Simons et al., 2012). An increase in students' academic growth has also been linked to participation in internships (Finley \& McNair, 2013; Jones, 2002; Simons et al., 2012).

Beneficiaries. There are three specific groups that seemingly benefit from internships: students, employers, and educational institutions (Gault et al., 2000). There are many psychological benefits that an internship experience has been shown to have on interns. For example, early research demonstrated that interns had a greater sense of responsibility for their own career development (Eyler, 1992; Hursch \& Borzak, 1979; Williams, 1990). For example, one former intern stated, each summer ended with me having gained a new understanding of what I did not want to do for a living. This was extremely important new knowledge about myself; and was made possible by three strong internship programs that were thoughtfully designed. (Greer, 2013, p. 72).

In terms of practical benefits, interns have reported gaining a better knowledge of the job market and the opportunity to build better networks (Groves, Howland, Headly, \& Jamison, 1977). As for educational institutions, Divine et al. (2007) suggest that one benefit comes in the form of an improved reputation. Gault et al. (2008) propose smarter students, various forms of funding, and networking to the local community as other forms of benefits as well. Practitioner input is also seen as a benefit to universities (Thiel \& Hartley, 1997). The benefits for employers range from receiving part-time help to better hiring decisions (National Association of Colleges and Employers, 2005). In retrospect, internships have many attractive qualities to all parties involved. 


\section{The Business Perspective}

Many employers and employees have concerns that noncognitive skills are poorly developed in current graduates (Scogin, Kruger, Jekkals, \& Steinfeldt, 2017). Employers are looking for employees that can think and problem-solve quickly, communicate effectively, and adapt accordingly. For example, over 400 U.S. employers ranked the following skills as most important for success in their industries: teamwork/collaboration, oral communications, professionalism/work ethic, and critical thinking/problem solving (Casner Lotto \& Barrington, 2006); these skills are not generated by solely mastering a single subject or degree area. These same employers rated their own recently hired employees as highly deficient in these areas. These findings suggest a disconnect between what modern-day businesses want in their employees and what academic institutional training provides (Scogin et al., 2017). Experiential learning activities, such as internship programs have been shown to increase critical thinking skills, develop teamwork, and contribute to higher order thinking skills (Cater \& Jones, 2014; Heinrich, Habron, Johnson, \& Goralnik, 2015; Ives \& Obenchain, 2006).

For example, students who have completed internships in business environments have reported an improvement in their communication skills (Knemeyer \& Murphy, 2002). Students in business environments have also reported an improvement in their jobrelated skills as well (Divine et al., 2007). Internship supervisors have reported that their student interns have displayed an improvement in key competencies such as ethical behavior, integrity, and professionalism (Shoenfelt, Kottke, \& Stone, 2012). Holding the belief that internships are intended to increase a student's market value, many employers 
focus on building valuable skills such as critical thinking and written and oral

communication (Maertz, Stoeberl, \& Marks, 2014).

\section{Competing with Working Professionals}

Actual work experience has been seen to benefit students in many ways. For example, tangible work experiences help prepare students for the rigor of the workplace (Rosenberg, Heimler, \& Morote, 2012). They also give students an opportunity to demonstrate the application of technical skills in practical settings (Jackson, Sibson, \& Reibe, 2014). These experiences have also been seen to motivate students (Hergert, 2009).

Experiential learning has incentive today where opportunities beyond postsecondary education have become increasingly competitive (Acai et al., 2014). Competition for jobs in today's economy has reached new heights. According to the National Association of Colleges and Employers, employers seek the following attributes in job candidates: leadership skills, written and verbal communication skills, problemsolving skills, ability to work in a team, and a strong work ethic (NACE, 2017). Most of these traits cannot be crafted by simply going to college and earning degree. A report from the Canadian Council on Learning (CCL) emphasizes that students can no longer necessarily compete in the world of working professionals by simply having a degree in hand; instead, students must find new ways of marketing themselves as individuals by developing skills that cannot be taught in a classroom (CCL, 2008). Utilizing experiential learning experiences for marketing purposes helps to increase a student's competitive edge. Experiential learners who have completed co-op placements or internships have found that they are more competitive in the job market after graduation and have a clearer 
idea of their career aspirations than their peers who have not yet worked in their field of study (Acai et al., 2014).

\section{Career-Oriented}

Career-related internships are a critical part of wise and well-informed decisionmaking regarding careers (Cunningham, Sagas, Dixon, Kent, \& Turner, 2005; Gault, Leach, \& Duey, 2010). Nonetheless, the attitudes of participants in internships may vary. For example, a study found that for students who completed internships in retail, positive attitudes toward retailing after internship were significantly related to their attitudes before the experience (Liu, Xu, \& Weitz, 2011). Contrastingly, in a study comparing interns and non-interns who were sports management majors, although there was no difference in attitude at the beginning of the internship, those students who participated in an internship had a less positive attitude toward the profession than did non-interns at the end of the internship (Cunningham et al., 2005). In another study, researchers reported that for some interns, occupational choices were in the process of being overturned as opposed to being refined (Walmsley, Thomas, \& Jameson, 2012). It can be surmised that even an unsuccessful internship experience can aid students in determining career direction and help them avoid unsuspecting career paths.

Career opportunities, such as job offers with the current company may develop as a result of participating in an internship. The experience of an internship may even help to facilitate a smooth transition from student to employee compared to students who have no experience in professional work environments (Narayanan, Olk, \& Fukami, 2010). Research supports the value that internships hold in helping students become aware of their vocational attributes. (Taylor, 1988; Drewery et al., 2016). 


\section{The Distance Learning Boom}

According to the Online Learning Consortium, the growth rate in distance education courses continues to exceed the overall growth rate in higher education (Allen \& Seaman, 2010a, 2010b, 2015). Distance education is institution-based, formal education where the learning group is separated but connected by interactive telecommunications systems. There are four components to the definition of distance education: institutionally based; separation of teacher and student; interactive telecommunications; and sharing of data, voice, and video (Simonson, Smaldino, \& Zvacek, 2015 p. 32).

Originally, distance learning programs were sought out by those who had no other means of completing their educational goals (Crawley, 2012). These students were usually adults ranging from age 25 and up, who were returning to school, and who also had other obligations outside of their studies to take care of. According to the National Center for Education Statistics (NCES, 2002), there are many characteristics that categorize a non-traditional student such as, but not limited to, delays in enrollment, parttime attendance, work weeks that consists of 35 hours or more, or has dependents. According to Crawley (2012), the demographics of distance learners range widely and bring with them a diverse set of needs as well. Simonson et al. (2015) reiterates that not only can distance learners be of any age, they can have reached any educational level and have a variety of educational needs.

\section{The Expansion of Distance Learning Opportunities}

Researchers have identified areas where experiential learning opportunities could be further developed or improved. One such area is the accessibility of experiential 
learning opportunities to a broad range of students (Acai et al., 2014). Some of the current face-to-face opportunities may require a considerable amount of scheduling flexibility, so non-traditional students may be at a disadvantage.

Since internship programs tend to be based on-site, it is beneficial for students to live within commuting distance to the program. This poses an extreme disadvantage for non-traditional students who do not live within commuting distance to the internship site. To participate in the internship, they are faced with several options: to travel long distances, to relocate temporarily, or to pass up the opportunity to participate in the internship altogether (Ruggiero \& Boehm, 2016).

Virtual internship opportunities come in various shapes and sizes. An internet search will reveal opportunities for working on open source projects, HTML development, and magazines and journals (Cornelius et al., 2008). One example of an advertisement is from the Central Europe Review,

[CER] works with university students from all parts of the world through our Virtual Internship Programme. All you need is access to a computer and a desire to learn," (CER, 2017). Virtual internships are also being used in teacher preparation programs (Heafner, Petty, \& Hartshorne, 2012).

There are many other organizations currently offering virtual internships as well, such as the US Journal of Academics (2017), who offers virtual internships to candidates who are interested in global marketing and new technologies. The School of Information at San Jose State University has a Professional Experience Internship Program that offers graduate and professional students the opportunity to complete a virtual internship for course credit and genuine work experience (SJSU, 2017). On another note, to help provide Indiana employers with students who are well-versed in online work as well as 
expose students to valuable work experience, Western Governors University (WGU) Indiana created a virtual, project-based internship program (WGU, 2017). The United Nations University also hosts a virtual internship program to engage students with work performed in the Information and Communications Technology Department (Hey Success, 2017). Sports Management Worldwide (SMWW) even offers a virtual program for undergraduate students who need to fulfill internship requirements for graduation (SMWW, 2017). The Job-search engines, such as internships.com (2017), LinkedIn (2017), and Intern Queen Inc. (2017) are also promoting virtual or remote internships with over 7,000 postings. Research has revealed many benefits through remote or virtual work including, a reduction in employer costs, an increase in employee satisfaction, and an increase in employee productivity (Ruth \& Chaudhry, 2008; Shin, El Sawy, Sheng, \& Higa, 2000; Weinert, Maier, Laumer \& Weitzel, 2014).

\section{Telecommuting}

Not only has virtual work been introduced into internship programs, but have been made popular by regular employees as well. In efforts to accommodate the 24-hour business cycle as well as to recruit the best talent available regardless of geographic location, companies have begun utilizing telecommuters, remote employees, and virtual workforces (WGU, 2017). Telecommuting is a work arrangement that allows an employee to work outside the office. An employee can choose to work from home or a location close to home, such as a library, coffee shop, or other location. Employees keep in touch with co-workers and employers through telecommunication links (i.e. email or phone) (Doyle, 2017). Telecommuting programs have gained popularity due to its assurance of a flexible work environment (The Viscardi Center, 2017). These programs 
have allowed employees an opportunity to remain productive without being in a physical workplace. A recent report on telecommuting in the U.S. Employee Workforce released the following statistics on telecommuters:

1. Between 2005-2015, telecommuting has increased from 1.8 million employees to 3.9 million employees

2. $2.9 \%$ of the total U.S. workforce works from home at least $50 \%$ of the time

3. The average annual income of telecommuters is $\$ 4,000$ higher per year than employees who work on site

4. The average telecommuter is 46 years of age or older

5. Compared to U.S. employers in $2010,40 \%$ more U.S. employers currently offer flexible workplace options (Flexjobs, 2017)

While traditional internships provide students with many benefits, they also pose challenges, especially to non-traditional students. Virtual internships offer many of the same benefits as traditional internships and reduce the challenges that are posed to nontraditional students, such as relocating or traveling long distances. Virtual internships have even been advocated as being a valuable addition to traditional education (Jeske \& Axtell, 2014).

\section{Virtual Effectiveness}

The main purpose of a virtual internship is to link the application of theoretical concepts learned in educational settings to a professional practice using telecommunication means only. The production of virtual internships has been on the rise over the past few years (Clemmons, 2015). A virtual internship allows an intern to work 
remotely using applications such as the phone and internet. An opportunity such as this would allow students who are geographically dispersed to participate in practical learning experiences. These types of internships initially started with small web-based companies, but are now popular with all kinds of businesses (Clemmons, 2015).

Results from a study disclosed that three out of every four companies surveyed have virtual workers on their payroll, and that the numbers of virtual workers employed by those companies is expected to double every year for the next 10 years (Mulki, Bardhi, Lassk, \& Nanavaty-Dahl, 2012). The virtual workforce has grown nearly $80 \%$ since 2005 and is expected to continue growing (Global Workplace Analytics, 2013). In the U.S., $2.6 \%$ of the employee workforce consider their homes to be their primary places of work (Ruggiero, \& Boehm, 2016). In 2013, 60\% of students were open to doing a virtual internship (Parcells, 2013); currently, over 70\% are open to the idea (WayUp, 2017). Due to the growing interest in the usage of virtual internships in higher education, many studies have explored their effectiveness (Fyfe \& Dennett, 2014; Ruggiero \& Boehm, 2016; Straus, Johnson, Marquez \& Feldman, 2013).

According to Bayerlin (2015), the overall success of a virtual internship is correlated to how much a student perceives it to reflect real situations. He advocates that virtual internships should be viewed as teaching models that prepare students for practical work. The connective learning model of Guile and Griffiths (2001) is outlined by Bayerlin (2015) in a virtual internship model for accounting programs to reproduce the benefits of practical work experiences in virtual environments. Through this model, a student's technical knowledge and workplace-related skills are developed by framing peer-interactions, engaging with resource materials, and maintaining a relationship with 
the academic institution while in an immersive workplace simulation. It is thought that the use of a "non-threatening" environment to host these internships will better enable students to explore everyday work challenges while receiving mentorship from the academic institution and peer-support from their fellow student interns.

Challenges. Challenges associated with the use of virtual internships in higher education have also been identified, such as student guidance and support. It is suggested that the objectives, activities, and evaluation procedures with regards to virtual internships should be well aligned. (Ahsan \& Hassan, 2013).

Albeit, research supports many benefits through the use of virtual internships, such as the development of relationships (Ruggiero \& Boehm, 2016). Virtual internships have also been shown to have the potential to inform project-based learning (Fyfe \& Dennett, 2014).

\section{Virtual World Examples}

Research shows an emerging trend in the use of virtual platforms for experiential learning programs over the past decade. For example, researchers found that project success in a virtual internship was related to the co-construction of knowledge between the interns, their mentors, and their clients (Ruggiero \& Boehm, 2017). This study consisted of virtual interns from 11 different university programs who were asked to apply their academic experiences in constructing projects for clients while under the supervision of mentors.

Another example highlights a structured role-playing web-based simulation exercise on Mekong Basin environmental management challenges is used as one of a two-part linked approach to an experiential learning program for students with social and 
physical science backgrounds in Australia and Asia (Hirsch \& Lloyd, 2005). This simulation exercise runs over four weeks and follows a five-week field-based learning exercise. The web-based simulation was deemed necessary to afford students the opportunity to learn the development geography of a remote location.

High school students in California's Oakland Unified School District participate in virtual medical internships as part of their school curriculum (Meyer, 2016). The school district follows a linked learning model, which not only allows students to take a sequence of courses specific to their chosen career, but also allows them to participate in experiential learning opportunities. The district has been able to partner with local businesses, organizations and government to provide their students with those experiences. This virtual medical internship is a computer-based simulation that is used to prepare the students for in-person internships.

The University of Wisconsin developed a virtual internship course for their firstyear engineering students to provide a platform for effective learning that can accommodate a large group of students at a low cost (Chesler, Arastoopour, D’Angelo, Bagley, \& Shaffer, 2013). Students in the course are split up into teams that are guided through multiple design-build-test cycles by experienced mentors in a simulated environment. This internship course is an example of a Massively Adaptive Complex Realistic Online Simulation with Interactive Mentoring (MACROSIM). These MACROSIMs engage students with realistic problems that require innovative solutions along with live mentors who interact with them to guide the learning process (Chesler et al., 2013). 
Researchers developed a real-time online observation (ROGI) program to facilitate an online graduate teaching internship program (Heafner et al., 2012). ROGI is used to observe and communicate with the interns remotely. ROGI's goal was to create a cost-effective alternative to face-to-face observations for teacher preparation programs. The use of ROGI resulted in an extension of university outreach to remote geographic locations across the state. Darius, Portier, and Schrevens (2007), and Nolan and Temple Lang (2007) have examples as well of open sourced virtual environments that have been used as substitutes for the physical world in statistics education.

While many of these programs have been evaluated for effectiveness and satisfaction, there is a lack of exploration on the leadership behaviors of the supervisors involved. More specifically, there is a lack of curiosity in the decisions supervisors make and how they may or may not help virtual interns learn and succeed in these environments.

\section{PGTL Revisited}

According to research, leadership refers to a supervisor's style and behavior in relation to setting the goals and the agenda for a team (Dansereau et al., 1995; Gu, He, \& Liu, 2017). Leadership also includes the supervisor's ability to develop the team and the team's culture (Dansereau et al. 1995). Researchers (Afshari, Siraj, Ghani, \& Afshari, 2011) indicate that the concept of leadership should mainly encompass the interpretations regarding the supervisor's behavior patterns and the influence on subordinates' activities. Regarding virtual internships, this would involve the way supervisors supervise virtual interns. If experiential learning opportunities are to be transformative experiences for 
students, then an evaluation of supervisory influences on interns' activities should be taken into consideration.

PGTL assumes that leaders are flexible in that they can change their behavior or style, depending upon the situation (Clark, 2013). Considered a dyadic approach to leadership, PGTL focuses on the relationship between a leader and a subordinate or follower (Yukl, 2013). This theory can be thought of as a process in which a leader selects specific behaviors that are best suited to the subordinate's needs and the working environment so that the subordinate can be guided through a path in the attainment of daily work activities or goals (Northouse, 2013). While PGTL is not a detailed process, it generally follows these basic steps (Clark, 2013):

1. Determine the employee and environmental characteristics

2. Select a leadership style

3. Focus on motivational factors that will help the employee succeed

\section{Origin}

PGTL was originally developed to accommodate prior findings from empirical investigations of the effects of leader task orientation and leader person orientation on subordinate satisfaction and performance (House, 1996). Prior to the introduction of the theory, the most frequently used measures for task and person orientation were the Ohio State leader initiating structure and leader consideration scales (Stogdill \& Coons, 1957). Consideration focused on relationship behaviors, such as respect and trust. Initiating structure focused on task behaviors, such as organizing, scheduling, and seeing that work is completed (Stogdill, 1974). The results from these measures were increasingly mixed; 
some studies showed positive relationships between the two variables, negatives relationships, or no relationships at all (as cited in House, 1996).

It was not until the publishing of Martin Evans' paper in 1970 that the idea for PGTL came to fruition. Evans's (1970) research evaluated the relationships between the Ohio State measures of leader consideration and leader initiating structure and follower perceptions of path-goal relationships. This research found support for the hypothesis that the two leader behaviors would be positively related to follower path-goal perceptions in one organization, but not in a second organization. Basically, the effects of task and person oriented leadership behaviors were contingent upon the organizational context in which the leaders and followers worked. House (1996) suggests that the relationship between structure and subordinate satisfaction and motivation is contingent on the degree to which subordinates need clarification of the behaviors required of them to perform effectively.

Initial version of PGTL. "The motivational function of the leader consists of increasing personal payoffs to subordinates for work-goal attainment and making the path to these payoffs easier to travel clarifying it, reducing roadblocks and pitfalls, and increasing the opportunities for personal satisfaction en route" (House, 1971, p. 324).

Later version of PGTL. House and Mitchell (1974) advanced two general propositions in this version:

1) Leader behavior is acceptable and satisfying to subordinates to the extent that the subordinates see such behavior as either an immediate source of satisfaction or instrumental to future satisfaction 
2) Leader behavior is motivational to the extent that (a) such behavior makes satisfaction of subordinate's needs contingent on effective performance and (b) such behavior complements the environment of subordinates by providing coaching, guidance, support and rewards necessary for effective performance (p. 84).

Reformulated theory. House (1996) proposed a reformulated path-goal theory of work unit leadership. This reformulated theory specifies leader behaviors that empower subordinates as well as provide satisfaction and promote work unit and subordinate effectiveness. The theory broadens the seminal theory to include effects of leaders on subordinates' ability to perform effectively as well as effects of leaders on work unit and individual subordinate performance (House, 1996). Research has found that the core of the reformulated theory is no different than the original one (Northouse, 2013). Considering the purpose for this study consists of exploring a supervisor's relationship with an individual, rather than a work unit, the earlier version of PGTL proposed by House and Mitchell (1974) will frame this study.

Underlying notion. The notion that underlies PGTL is that individuals in positions of authority will be effective to the extent that they complement the environment in which their subordinates work by providing the necessary cognitive clarifications for subordinates to experience intrinsic satisfaction through work-goal attainment (House, 1996). Subordinates need leaders who can provide what is needed for them to thrive in a work environment (Northouse, 2013). It is the leader's responsibility to create links where the environment lacks in clear causal linkages between effort and goal attainment. When a subordinate presumes linkages exist where there do not, the 
leader must clarify these presumptions. Most importantly, where subordinates lack adequate support or resources required to accomplish work goals, it is the leader's responsibility to provide the support and resources needed (House, 1996).

\section{Specific Leader Behaviors}

House (1971) described two general classes of leader behavior: path-goal clarifying behavior and behavior directed towards satisfying subordinate needs. Further research helped to operationally define these behaviors, along with two others, as part of the theory. In their research, House and Mitchell (1974) defined four kinds of specific leader behaviors: 1) Directive path-goal clarifying leader behavior; 2) Supportive leader behavior; 3) Participative leader behavior; and 4) Achievement oriented behavior. The directive behavior is based on Stogdill's (1974) initiating structure scale, while the other three behaviors are based on the consideration scale.

Directive path-goal clarifying leader behavior. This behavior is focused on providing structure for subordinates (House, 1996). It emphasizes giving subordinates directions regarding their tasks, clarifying performance expectations, and outlines rules and regulations (Yukl, 2006).

Supportive leader behavior. Use of this behavior is seen to reduce stress and alleviate frustration for subordinates (House \& Mitchell, 1974). Supportive leader behavior is also expected to increase performance of subordinate behaviors that are contingent on goal-directed efforts (House, 1971).

Participative leader behavior. Participative leader behavior encourages subordinates to participate in decision-making on work operations (House, 1996). 
Leaders who use this behavior consult with subordinates and take their opinions into account when making decisions.

Achievement oriented behavior. Leaders who are concerned with raising confidence levels in subordinates and inspiring them to meet challenging goals utilize this behavior. Leaders sometimes offer up rewards as incentives for meeting goals. Achievement oriented behavior encourages performance excellence in subordinates and pushes subordinates to strive for higher standards (House, 1996).

\section{Two Distinct Types}

Correspondingly, the literature has differentiated leadership styles into two distinct types: supportive and directive leadership (Gu et al., 2017; Yukl 2006; Wendt, Euwema, \& Hetty van Emmerik, 2009). These two types have been helpful in exploring the various roles of different leadership styles (Mannheim \& Halamish 2008).

Supportive leadership is defined as the supervisor's relationship-oriented behavior (Gu et al., 2017). It emphasizes satisfaction of subordinates' needs and preferences, the care for subordinates' welfare, and the creation of a friendly and psychologically supportive work environment (House 1996). More specifically, supportive supervisors exhibit concern for the feelings and needs of their subordinates.

By contrast, directive leadership mainly reflects the supervisor's task-oriented behavior (Gu et al., 2017). This leadership style has a focus on controlling discussions, dominating interactions, and personally directing task completion (Cruz, Henningsen, \& Smith 1999). Directive leadership normally focuses on getting the work done by giving commands directly or setting deadlines. 
While the research supports two distinct types of leadership styles, it is quite possible that supervisors in virtual internship programs may exhibit any one of the four specific behaviors defined by PGTL.

\section{Variables}

There are certain variables associated with contingency theories; one predictor variable, one dependent variable, and one or more situational variables (Yukl, 2013). Concerning PGTL, the predictor/independent variables are the leader behaviors (House, 1996). Independent variables are usually described in categories, such as task and relations behavior, while dependent variables surround subordinate satisfaction or performance (Yukl, 2013).

Situational variables concern themselves with conditions that the leader is unable to change in the short term, such as characteristics of work, characteristics of the subordinates, and characteristics of the leadership position (Yukl, 2013). In the case of virtual internships, the conditions would include virtual working environments, students who are college-level or above, and a variety of supervisory roles.

Subordinate characteristics. Regarding subordinate characteristics, there are four that researchers have focused on: needs for affiliation, preferences for structure, desires for control, and self-perceived level of task ability (Northouse, 2013). For example, subordinates who have a higher need for affiliation tend to prefer supportive leadership, while subordinates who tend to be dogmatic, prefer directive leadership (Vasella, 2011).

Task characteristics. Task characteristics can include the organization's formal authority system, the subordinate's work group, and the specific task design (Northouse, 
2013). These characteristics are seen to have a major effect on how subordinate satisfaction and motivation is affected by leader behaviors (Vasella, 2011).

\section{How does it work?}

PGTL assumes effective leaders are those who meet subordinates' needs (Vasella, 2011). They are those who assist subordinates in removing obstacles and in achieving their goals. They are also those who provide structure, support, and guidance to subordinates. Effective leaders are those who are flexible enough to use behaviors that are best suited to meet their subordinates' needs. These leaders must have the skill and ability to recognize when different leadership behaviors need to be used to influence their subordinates (Lian \& Tui, 2012).

\section{eSupervision}

Leader effectiveness is mostly evaluated in terms of the outcomes of a leader's influence attempts on individuals, teams, organizations (Yukl, 2013). Tourman et al. (2012) note many variables that can influence professional learning in the workplace, such as the tasks that are given, their degree of difficulty, and the nature of help offered by the supervisor. There are personal responsibilities and roles in the virtual supervisory process that both the eIntern and eSupervisor must recognize. For instance, they must both participate in the virtual internship on the basis of shared mission, commitment to the profession, and mutual respect. Researchers recognize one important indicator of leader effectiveness to be the extent to which a follower's performance is enhanced and goal attainment is facilitated (as cited in Yukl, 2013). Effective supervision has been linked to student development and overall learning outcomes (Sweitzer \& King, 2014). Attitudes and perceptions of the leader, which are usually measured by interviews or 
questionnaires, are other common indicators of leadership effectiveness (Yukl, 2013).

The following are characteristics that make for effective eSupervisory relationships.

Communication. Effective eSupervisory relationships consist of frequent communication between the eIntern and eSupervisor. Neukrug (2012) states that the communication must be one of mutual respect and based on humanistic principles (empathy, understanding, trustworthiness, etc.).

Feedback. Effective eSupervisory relationships consist of constructive and objective feedback given by the eSupervisor on the eIntern's job performance. This feedback should focus on development and the mastery of specific skills. The availability of feedback should allow the eIntern to discuss any concerns about development and to obtain support for any challenges or obstacles faced (Cicco, 2014).

Satisfaction. Effective eSupervisory relationships are defined by the attitudes of eInterns and eSupervisors regarding the intern's professional growth and development (Cicco, 2014). This includes rapport and communication as well.

While virtual supervisory relationships provide many advantages, there are some concerns to be made aware of such as providing immediate support for interns and maintaining confidentiality (Vaccaro \& Lambie, 2007).

Examples of virtual supervision. Schwartz-Bechet (2014) examined teachercandidate preparation programs and compared two different models of virtual supervision to identify best practices for the use of information and communication technology in the supervision of student teachers and interns. The study was qualitative in nature with supporting quantitative data from the teacher-candidates along with their eSupervisors. The best practices were identified as: communication, monitoring, management, 
reflection, and engagement. Providing feedback and developing open lines of communication were significant aspects. The study also identified that there is a need to educate eSupervisors regarding how to better utilize virtual technologies and to also assist them in understanding the benefits to themselves and the teacher-candidates who are taking part in this process.

In the ROGI program mentioned earlier, the eSupervisor observes the eIntern remotely utilizing a multimedia conferencing platform for 60-90 minutes. In this process, the eIntern wears a wireless headset (for audio capabilities) and strategically places a webcam (for visual capabilities) in the classroom so that the eSupervisor can see a most of the students. After the observation is complete, both the eIntern and eSupervisor participate in a post-conference debriefing to provide feedback and give time for reflection (Heafner et al., 2012).

\section{Administrative Challenges}

Administrative challenges may make institutionalizing experiential education programs difficult. For example, administrative demands, such as requirements for productivity in research or larger class sizes, may complicate the ability of institutions to institutionalize experiential learning programs (Austin \& Rust, 2015). Researchers (Donovan, Porter, \& Stellar, 2010) provide several strategies for successful experiential education programs such as defining experiential learning, engaging faculty in planning and oversight, developing learning goals, establishing some type of quality control (i.e., course review), developing communities of practice, seeking "inside/outside expertise" (i.e. bringing in speakers or attending conferences) and showcasing student work (p. 93). 


\section{Proper Planning}

The National Society for Experiential Education (NSEE) offers several principles of good practice that should be considered in the development of an experiential education program: intention, preparedness and planning, authenticity, reflection, orientation and training, monitoring and continuous improvement, assessment and evaluation, and acknowledgement (NSEE, 1998):

Results from an evaluation of the experiential learning literature can be used by universities/colleges to define and begin to operationalize experiential learning programs, to understand the values of experiential learning to the learning process and to provide insight into how these programs can be institutionalized (Austin \& Rust, 2015, p. 146).

It has been said that what is needed in our understanding of high-impact practices is a "more advanced logic model that will allow us to document the relative importance and influence of the structural and programmatic characteristics of high-impact practices in terms of inducing student effort and other desirable outcomes" (Kuh \& O’Donnell, 2013, p. 8).

\section{Summary}

Virtual internships are experiential learning opportunities that can help a broad range of students become engaged with communities and the individuals that they are working with; which would create an opportunity for a more meaningful learning experience. However, a review of the literature shows that there is limited research on eSupervisory relationships with virtual interns and how these relationships inform us about the eSupervisors' learning contributions to society. The recognition and use of certain leadership behaviors in a virtual internship can aid in determining what forms of learning will take place. 


\section{CHAPTER THREE: METHODS}

The Virtual Student Foreign Service (VSFS) program is a virtual internship program for U.S. citizens, college-level and above, to work on projects of global importance throughout the U.S. government. VSFS aims to expand work opportunities to those students interested in exploring career interests, but who are otherwise bound by geographical means. Through this program, government agencies have access to a wide range of talented students who can aid in advancing the work of the government. VSFS is managed by the Office of eDiplomacy in the U.S. Department of State's Information Resource Management Bureau and has been in effect since 2009 (VSFS, 2017). With over 1,300 vacancies in more than 30 different agencies, VSFS is currently the largest virtual internship program in the world (Haiti Embassy, 2017; VSFS, 2017). The problem this study explored was the lack of research on how eSupervisory relationships with virtual interns inform us about the eSupervisors' learning contributions to society.

The U.S. Government Accountability Office (GAO) makes extensive use of case studies in their research and evaluations (Baskarada, 2014). GAO defines a case study as "a method for learning about a complex instance, based on a comprehensive understanding of that instance obtained by extensive description and analysis of that instance taken as a whole and in its context" (GAO, 1990, p. 15). Due to the interrelatedness of learning outcomes between multiple parties, however, a multiple case study analysis will be employed to collect information-rich data. Stake (2013) defines the multi-case project as a research design for closely examining several cases linked together. 
While qualitative case studies are widely used, their methods are not well understood (Baskarada, 2014). Bogdan and Biklen (2007) offer insight into the theoretical underpinnings of qualitative research: that it "produces an interpretation of reality that is useful in understanding the human condition" (p. 27). Moreover, qualitative research is based on a view that social phenomena and the nature of cases are situational (Lincoln \& Guba, 1985). The social phenomena, later referred to as a quintain (Stake, 2013), that was studied here was the learning that results from participating in a virtual internship and how it is influenced by supervisory styles. Qualitative research helps bring to light the meaning of what people have constructed about their experiences (Rubin \& Rubin, 2012; Merriam, 2002). In an effort to clarify the methodology behind this research as well as provide a structural foundation for it, Yin's (2009) six-stage case study process was utilized. While Yin's six-stage process was utilized to organize this study, it is Stake's (2013) suggested methods that were used for the analysis. Yin's (2009) six stages are as follows: plan, design, prepare, collect, analyze, and share.

\section{Plan}

As stated above, the problem this study explored was the lack of research on how eSupervisory relationships with virtual interns inform us about the eSupervisors' learning contributions to society. For the purpose of aligning the terminology with that of which is used in VSFS, virtual interns will be referred to as eInterns and supervisors will be referred to as eSupervisors from this point forward. The purpose of this study was to analyze to certain leadership methods that eSupervisors were utilizing with eInterns and how these methods informed us about learning outcomes. 


\section{Sample}

As VSFS allows for any U.S. citizen, college-level or above to participate, only eInterns who were involved in a noncredit earning internship and did not have university supervisors were used for this study. This specification aided in reducing any confounding data from eInterns who have faculty supervisors and eInterns who do not have an additional faculty supervisor.

\section{Research Questions}

The following research questions were examined:

1. How do eSupervisors describe their delivery of educational contributions that promote learning in a virtual internship environment?

a. What do eSupervisors want their eInterns to learn during their virtual internships?

b. How are eSupervisors helping eInterns accomplish their learning goals?

2. How do eInterns interpret the educational contributions that they receive from their eSupervisors into learning experiences?

a. What do current eInterns wish to learn during their virtual internships?

b. What do current eInterns perceive they are learning during their virtual internships?

3. How do virtual internships deliver educational contributions that add value to an eIntern's post-internship experience?

a. How do past eInterns describe what they learned during their virtual internships? 
b. How do past eInterns describe how they are applying the skills they have learned from their virtual internship experience?

\section{Rationale}

According to Yin (2009), how and why questions are better answered through case studies. Research also shows support for case studies in generating insight on certain systems or functions in society, such as program administration and public support (Parlett \& Hamilton, 1976; Stake, 2013). As mentioned in previous chapters, PGTL outlines the theoretical framework for this study. Characterized by how and why research questions, these types of studies explore the relationships that are proposed between different theory components (Yin, 2009).

The selection basis that was used for this study was based on special interest. Special interest selections are probabilities chosen based on special or unusual attributes (GAO, 1990). In multi-case studies, "we study what is similar and different about the cases in order to understand the quintain better" (Stake, 2013, p. 6). In this study the quintain refers to a phenomenon involving multiple parts in diverse settings The cases were selected based on the availability of participants and on the diversity of the demographic combinations. Due to the virtual context in which the study took place, this study was less focused on the demographic relationships and more focused on the learning outcomes. eSupervisors and the eInterns they were currently supervising were studied, along with eInterns whom they have supervised in the past.

\section{Research Paradigm}

The underlying research paradigm for this study was critical realism. In scientific research, paradigms are a set of common beliefs between scientists about the nature of 
problems and the methods used to research them (Kuhn, 1962). According to research, critical realism takes a meta-theoretical position in the form of a reflexive stance concerned with "providing a philosophically informed account of science and social science which can in turn inform our empirical investigations" (Archer, Decoteau, Gorski, Little, Porpora, Rutzou, Smith, Steinmetz, \& Vandenberghe, 2016, para 2). Critical realism argues for a structured and differentiated account of reality in which difference, stratification and change is central (Bhaskar, 1975). This paradigm supports viewing the process of achieving educational outcomes as a system with interrelated parts that are working together to achieve the same goal. In the case of this study, those parts were the eSupervisors and the eInterns. By selecting cases based on special interest, this study was able to examine the various attributes that contributed to the educational outcomes of the eInterns. Researchers have advocated for critical realism as the preferred paradigm in case study research (Easton, 2010).

\section{Design}

\section{Units of Analysis}

For the 2017-2018 program, VSFS had a total population of 1300 eInterns and 822 eSupervisors. Four eSupervisors from the target group were selected based on information provided by key informants at the research site. Stake (2013) cites three main criteria for selecting cases:

1 . Is the case relevant to the quintain?

2. Do the cases provide diversity across contexts?

3. Do the cases provide good opportunities to learn about complexity and contexts? (p. 23) 
Correspondingly, Yin (2009) states that each case in multi-case studies should be selected so that it predicts either similar or contrasting results. As previously mentioned, the cases were selected based on the availability of participants and on the diversity of the demographic combinations. The sampling strategy that was used for this group was intensity sampling. An intensity sample consists of "information-rich cases that manifest the phenomenon of interest" (Patton, 1990, p. 171). For example, eInterns who have good working relations with their eSupervisors and eInterns who have bad working relations. This type of sampling required some prior information from key informants and substantial judgement from the researcher. Intensity sampling is a strategy of purposeful sampling. Purposeful sampling is used in qualitative research so that the researcher can intentionally select individuals to understand the central phenomenon (Creswell, 2015). For each eSupervisor, two current eInterns who were participating in a noncredit earning internship were selected as well as two eInterns who have already completed the program. For the purposes of this study, those eInterns who have already completed the program were referred to as graduates and the eInterns who were currently in the program were referred to as students. Each group accounted for a single case with multiple units of analysis: one supervisor, two students, and two graduates. Therefore, totaling the number of single cases to four. Stake (2013) advocates that no fewer than four cases or more than 10 should be used when conducting multi-case studies; he states, "two or three cases do not show enough of the interactivity between programs and their situations, whereas 15 or 30 cases provide more uniqueness of interactivity than the research team and readers can come to understand" (p. 22). There was a total of 20 participants in this study. 
Recruitment. Consent from the program manager was obtained to distribute the recruitment materials to the sample participants identified by key informants.

Recruitment materials for this study included an informational letter and consent form to contact participants via text messaging. Both items were distributed through email. The Qualtrics survey platform was utilized to distribute the recruitment materials. Once consent to contact participants via text messaging was been obtained, contact included text messages as well as emails and phone calls. A link to the informational letter was embedded in the email along with a link to an informed consent check-out page. Once informed consent was been obtained, the researcher proceeded with scheduling and conducting the interviews.

\section{Multi-Case Study Design}

According to Stake (2013), researchers undertake multi-case studies to study a phenomenon involving multiple parts in diverse settings. These phenomena are referred to as quintains. The quintain in this study was the learning that results from participating in a virtual internship and how it is influenced by supervisory styles. It is suggested that to carry out the study of the quintain, the data gathering and reporting of individual cases should be organized separately (Stake, 2013). As such, each case was gathered and analyzed separately before conducting a cross-case analysis.

Procedures. The procedural steps for conducting this study were as follows:

1. Obtain proper permission to conduct the study from research site

2. Obtain approval from FIU Institutional Review Board

3. Identify sampling frame

4. Consult with key informants for purposeful sampling 


\section{Conduct pilot study}

6. Gather, transcribe, and analyze results. Data collected was stored in an encrypted file on an encrypted computer that only the researcher had access to. Data will be saved until May 31, 2020.

7. Refine data collection plans as necessary

8. Send out participation letter via email to potential participants from sample. Email addresses from a database listed provided by the Program Manager at the research site.

9. Upon acceptance of participation, schedule interviews through Zoom webconferencing to allow for interviews to be recorded.

10. Gather, transcribe, and analyze results. Data collected was stored in an encrypted file on an encrypted computer that only the researcher had access to. Data will be saved until May 31, 2020.

11. Conduct steps $8-10$ per case group

12. Conduct cross-case analysis

13. Report results

\section{Data Consideration}

Edmonds and Kennedy (2012) note that the quality of case studies depends on construct validity, internal validity, external validity, and reliability.

Construct validity. Constructs are the elements of information to be sought out by the researcher (Grovers et al., 2009). These elements of information are usually ideas that are presented verbally. The study's themes of focus are the same as the constructs: 
the eSupervisor's perceived relationship, style of leadership, and learning objectives with the eInterns in VSFS.

Leadership refers to a supervisor's style and behavior in relation to setting the goals for a team as well as the supervisor's ability to develop the team (Dansereau et al., 1995). This definition indicates that the concept of leadership should mainly encompass the analyses of the supervisor's behavioral patterns and the influence on subordinates' activities (Afshari, Siraj, Ghani, \& Afshari, 2011). Accordingly, research shows the existing leadership literature has differentiated leadership behaviors into task-oriented and relationship-oriented behaviors, which are also referred to as initiating structure and consideration, and has further categorized leadership styles into supportive and directive leadership (as cited in Gu et al., 2017). The constructs are defined below:

Supervisory style. Supervisory style is defined as the principles that govern the relationship between supervisor and supervisee, be they intended or unintended, explicit or implicit (Boehe, 2016). Social cognitive theory suggests that supervisory styles are critical external factors that may not directly influence individual behavior, but indirectly influence it through influencing psychological cognitive factors (Gu et al., 2017).

Supervisory relationship. Supportive leadership is defined as the supervisor's relationship-oriented behavior, with an emphasis on the satisfaction of subordinates' needs and preferences, the care for subordinates' welfare, and on the creation of a friendly and psychologically supportive work environment (House, 1996).

Supervisory objectives. Directive leadership mainly reflects the supervisor's taskoriented behavior, with a focus on controlling discussions, dominating interactions, and personally directing task completion (Cruz et al., 1999). 
Possible issues. Threats identified with construct validity include reactivity to the experimental situation, experimenter expectancies, and novelty and disruption effects (Baskarada, 2014). A strategy for improving construct validity includes using multiple sources of evidence, such as multiple participants (Yin, 2009). Employing multiple sources of evidence can contribute to construct validity by providing multiple measures of the same quintain. As such, multiple eInterns and eSupervisors were interviewed. A table was also created to keep track of the alignment of the research questions, theory components, and constructs during the analysis (see Table 1).

Internal Validity. Internal validity is concerned with justifying causal relationships. Some threats to internal validity include ambiguous temporal precedence, selection, and additive and interactive effects (Baskarada, 2014). The use of data source triangulation can minimize the threats to internal validity (GAO, 1990). Additionally, Tracy (2010) advises that researchers should provide enough detail that readers may come to their own conclusion about the scene, rather than telling the reader what to think; also known as providing a thick description. This is an important aspect of qualitative research that was included in the report to reduce the amount of influence a researcher's perspectives may have on the intended audience.

External Validity. External validity deals with the problem of knowing whether the findings are generalizable to other cases (Baskarada, 2014). Threats to external validity include interaction of the causal relationship with units, outcomes or settings (Shadish, Cook, \& Campbell, 2002). Due to the qualitative nature of this study, external validity was difficult to achieve. Baskarada (2014) cites that case studies are generalizable to theoretical propositions and not to populations or universes. 
Reliability. Reliability is concerned with demonstrating that same results can be obtained by repeating the data collection procedure (Baskarada, 2014). One strategy for ensuring reliability of case studies includes the creation of a case study protocol (Yin, 2009). The case study protocol for this research project can be found in Appendix A. The case study protocol contributed to the reliability of this study by structuring and standardizing the investigation. Another way reliability can be displayed is through a

method of transparency as described by Tracy (2010). Transparent research discloses the study's challenges and any unexpected twists or turns. For example, one challenge that occurred during data collection was a low internet connection during several scheduled interviews which would not allow the researcher to conduct the web-conferenced interviews with her laptop. After switching to the mobile application on her phone, the researcher discovered that the interviews could not be recorded through the mobile app. This resulted in four interviews that were not recorded. Thankfully, the researcher caught the mishap early on and was able to take detailed notes during the interviews.

\section{Ethical Issues}

The most important ethical issues to be considered are obtaining informed consent and maintaining confidentiality of the participant's responses (Grovers et al., 2009). Subscribing to a pledge of confidentiality and restricting access to data to only those who have taken the pledge was one method reducing the risk of disclosure. Also, following the guidelines presented by the Common Rule (Grovers, et al., 2009, p. 379) helped to give participants meaningful control over information about themselves by making sure their rights were protected throughout the research process. These participants were asked to conduct interviews through the Zoom (2018) web-conferencing tool to allow for the 
audio from the interviews to be recorded. Since all participants were in a virtual internship and already used to using technology, there were minimal levels of negative impact on the interview process. In fact, the only negative occurrence was on the researcher's side when it came to switching to the mobile app to record the interviews. Participants were also made aware that the interviews were being recorded for analyses purposes, but would be safely stored along with the use of pseudonyms for their identities. Please note, there were no video recordings during these interviews; just audio only.

Subsequently, there were no direct benefits to individual participants. Risks for participating were minimal, meaning the risks were not thought to be greater than other potential participants experience every day.

\section{Prepare}

Prior to collecting any data for this study, approvals needed to be obtained from the dissertation committee, research site, IRB, and participants. After all approvals were obtained, the researcher began conducting the study. The first step, was to conduct a pilot study.

\section{Pilot Tests}

The purpose of conducting the pilot tests were to see if the interview protocols would be effective in answering the research questions. The tests were conducted with one eSupervisor, on student, and one graduate from the study. The researcher concluded that the protocols were effective in generating data that would answer the research questions after the pilot tests were conducted and the results were analyzed. Also, the researcher did not make any adjustments to the interview questions as the qualitative 
nature of the study and the semi-structed interview protocol allowed for follow-up questions to be asked if deemed necessary.

Conducting pilot tests for this study was useful in testing the research instruments and determining if any changes need to be made to the protocols. After the pilot tests were conducted, the researcher proceeded to collect data from the other cases.

\section{Collect}

To triangulate the study and increase its validity, interview data were collected from a variety of sources within each case. Interviews in case studies collect direct evidence about events and behaviors, while surveys capture perceptions (Yin, 2009).

The interviews for each case were conducted in the form of focused interviews. Focused interviews allow the researcher to refocus the questions or prompt for more information if something novel emerges during an interview (Baskarada, 2014). These types of interviews are more flexible than structured interviews and allow the researcher to better understand the perspectives of the participants (Dane, 2010; Daymon \& Holloway, 2002). The interview protocol for each unit of analysis in the case is outlined in Appendices B, C, and D.

In accordance with the case study protocols, multiple participants were interviewed to provide data for this study. Most of the interviews were audio-recorded and then transcribed, verbatim. A case study database was used to store relevant documents, such as transcripts, case study protocols, and reflexive notes from the researcher. All printed and written documents were scanned and then filed into the database. Interview recordings were uploaded into the database as well. The case study 
database was securely kept in a locked file on the researcher's desktop machine at all times.

\section{Data Collection}

After proper permissions were obtained, the researcher began with data collection for the pilot study phase. Data collection for this study began on January $16^{\text {th }}, 2018$. Multiple sources of data were collected in the form of interviews within each case. All interviews were conducted virtually through a cloud-based web-conferencing tool called Zoom (2018), to allow for audio recordings. Each case was made up of one eSupervisor, two students (current eInterns), and two graduates (past eInterns) who worked with the eSupervisor.

The intent of conducting the pilot study was to test the research instruments and determine if any changes needed to be made before collecting data for the other cases. Therefore, the first interviews conducted within each group (eSupervisors, students, and graduates) formed the pilot tests for this study instead of the pilot study being conducted on one single case.

There were a total of 20 interviews conducted and analyzed for this study. Due to the distinctiveness of projects offered within VSFS, the names of the specific agencies in which each participant worked and certain project details will not be revealed in an effort to prevent participants from being identified. Coded names will be used to refer to the participants as well to protect their privacy.

\section{Sample Demographics}

While demographics were not used during the analysis, there are presented here to present a descriptive overview of the participants in the study. The sample population 
consisted of 4 eSupervisors, 8 students, and 8 graduates from the VSFS program. Descriptive statistics for the total sample population are as follows: There were 14 females (80\%) and 6 males (20\%) who participated; 8 participants (40\%) fell between the 18-21 age range, 8 participants (40\%) were between $22-25,1$ participant (5\%) was between 26-30, 2 participants (10\%) were between 31-40, and 1 participant (5\%) was over the age of 61 . With respect to race, there were 10 White, non-Hispanics $(50 \%)$, 2 Blacks/African Americans (10.0\%), 4 Hispanics (20\%), 3 Asian/Pacific Islanders (15\%), and 1 Other (5\%). Those participants who were still taking classes were enrolled in universities all over the country, in states such as California, Iowa, Ohio, Michigan, Texas, and Virginia. There were even two students who were studying abroad in Canada and India.

\section{Analyze}

According to Stake (2013), contexts within a case need to be studied and described, whether evidence of influence is found or not. It is a researcher's task to review these contexts in search of correlated activities. To gain assurance that key meanings within cases are not being overlooked or that the researchers are not reading too much into what they see, important interpretations need to be triangulated (Stake, 2013). Triangulation is used in research to confirm that interpretations of data are accurate. As each case was comprised of an eSupervisor and four eInterns who have working relations with that eSupervisor, the grounds for triangulation were obtainable.

To avoid misinterpretation of the data, Stake (2013) suggests to listen to the interview recordings multiple times and from different perspectives as well. The researcher first listened to the recordings from all of the groups separately, then went 
back and listened the recordings from each case. This aided the researcher in generating alternate interpretations of the data.

Coding. Coding is an act of translation and an act of summarization (Grovers et al., 2009). Data analysis for the qualitative data consisted of first transcribing the interviews, then encoding all data into the NVivo Windows PC software program, and finally using a set of worksheets (Appendix E) developed by Stake (2013). The researcher used a free online transcription tool, Transcribe (2018), to transcribe all of the recorded interviews from speech to text.

Coding was performed at different levels of abstraction (Baskarada, 2014). First, each participant's responses were analyzed separately to discover certain characteristics relating to their backgrounds, experiences during the virtual internship program with their eSupervisors, and how the experiences were informed by their personal opinions. The researcher utilized a predesigned coding system based on the constructs of this study: eSupervisory Style, eSupervisory Relationship, and Learning Outcomes. The initial codes, expanded codes, and final codes generated for each major theme in this study are listed in Tables 2-4. Next, an analysis was conducted to display the similarities and differences between each participant's experience within the bounded system of each case. Then, a cross-case analysis was conducted to explore the quintain. As the amount of data received from each case was considered to potentially be overwhelming, a set of worksheets (Appendix E) developed by Stake (2013) were utilized to facilitate the crosscase analysis. These worksheets helped to organize the data and assure that the multi-case was being analyzed appropriately. The process for utilizing the worksheets is described in 
the cross-case analysis section. Finally, broad themes were generated that relate to the case.

Cross-case analysis. As mentioned before, the quintain in this study was the learning that results from participating in a virtual internship and how it is influenced by supervisory styles. First, findings originated after analyzing the single cases. Then, themes originated from the findings of the single cases to study the quintain. While the themes preserved the main research questions, the findings preserved the special circumstances of the cases. Both the findings and the themes were used as equal forces to understand the quintain (Stake, 2013). The quintain was then studied through a cross-case analysis. The quintain can be characterized by either uniformity or disparity, based on evidence from the single cases (Stake, 2013). The final report on the cross-case analysis presents the most important findings from each case as an assertion.

The worksheets (Appendix E) are not intended to present findings to the reader, but rather to facilitate a cross-case analysis. Worksheet 1, which presents an outline for gathering data for cases, was not used in this study. Worksheet 2 aided in monitoring the study's primary themes while reviewing data in each case. These themes originated from the research questions, but evolved as research for the case progressed. The themes were listed in the order that was thought to provide the most value to the quintain (Stake, 2013). Important case findings were gathered on Worksheet 3; there was one worksheet for each case. Worksheet 4 was used to rate the individual cases according to their usability in developing each theme.

Stake (2013) proposed three different tracks for analyzing the findings across cases: individual case findings (Worksheet 5A), merged findings (Worksheet 5B), and 
factors for analysis (Worksheet 5C). Track 1, individual case findings, emphasizes the various situations and findings of each case. Track 2, merged findings, emphasizes the multi-case. Track 3, factors for analysis, emphasizes quantitative analysis and places little focus on the situation of the cases. Merging the case findings was seen to be to most applicable to this study as each case was bound within the same virtual internship program; therefore, Worksheet 5B was utilized. Administrators of this program may find the results of this study useful when designing future programming or recruiting new eSupervisors. Data from Worksheets 2-4 was used to inform Worksheet 5B. This worksheet created a theme-based matrix of the findings and their importance from each case. Worksheet 6 was used to generate assertions about each case based on the information from Worksheet 5B. Finally, Worksheet 7 was used to aid in organizing the display of the results in the final report.

Process for analysis. The following steps below outline the researcher's process for analyzing the findings:

1. Analyze research questions and align them with questions from interview protocols (See Table 1).

2. Group questions from interview protocols into initial coded categories

3. For each case, start with the shortest interview and sort relevant data into the initial coded categories

4. For each case, go through each category and assign expanded codes for each participant

5. Place codes into a table for analysis

6. Generate final codes to supplement themes 
7. Conduct cross-case analysis using a set of worksheets (Appendix E) developed by Stake (2013).

\begin{abstract}
Share
The share stage focused on defining the audience, composing textual and visual materials, and displaying enough evidence for the reader to reach his/her own conclusions (Yin, 2009). The data analyzed is presented in several ways that involve visual representations and direct quotes. A summary of results, along with a conclusion is also presented.
\end{abstract}

\title{
Researcher's Role
}

The researcher's role and background with this study was an intricate one involving multiple stages. While the researcher was not an explicit participant-researcher for this study, her participation in other internships helped to generate background information on the topic that was useful in deciphering and understanding the quintain. Initially, the researcher worked with undergraduate students who were enrolled in fully online programs and became interested in virtual working environments through her desire to connect these students with more learning opportunities. Subsequently, the researcher participated in two face-to-face internships during her time spent conducting the study. These experiences allowed the researcher to utilize multiple perspectives throughout the entire process of conducting the interviews, analyzing the data, and then discussing it afterwards.

\section{Summary}

A multi-case qualitative case study was performed on a virtual internship program to examine eSupervisory relationships between eInterns and their eSupervisors. The 
sample population consisted of eSupervisors, current eInterns, and past eInterns from an existing virtual internship program in the U.S. The researcher's objective was to explore how certain leadership methods that eSupervisors are utilizing with eInterns are affecting their learning outcomes. 


\section{CHAPTER FOUR: RESULTS}

A multi-case qualitative case study was performed on a virtual internship program to examine eSupervisory relationships between eInterns and their eSupervisors. The introduction of virtual internships in experiential learning have expanded the amount of learning opportunities available to students and have been beneficial to the organizations that employ them as well. This study analyzed the overall intent of supervisors when it came to supervising virtual interns. The purpose of this study was to explore certain leadership methods that eSupervisors are utilizing with eInterns through the theoretical lenses of House's (1971) path-goal theory of leadership and the effectiveness of these methods on learning outcomes. For the purposes of this study, those eInterns who have already completed the program were referred to as graduates and the eInterns who were currently in the program were referred to as students.

Each group accounted for a single case and is presented as such in a case-by-case context: one eSupervisor, two students, and two graduates. The following steps below outline the researcher's process for analyzing the findings:

1. Analyze research questions and align them with questions from interview protocols (See Table 1)

2. Group questions from interview protocols into initial coded categories

3. For each case, start with the shortest interview and sort relevant data into the initial coded categories

4. For each case, go through each category and assign expanded codes for each participant

5. Place codes into a table for analysis 
6. Generate final codes to supplement themes

7. Conduct cross-case analysis using a set of worksheets (Appendix E) developed by Stake (2013).

Next, the results of the cross-case analysis are organized according to each major theme in this study: Supervisory Style, Supervisory Relationship, and Learning Outcomes. The findings from each case were populated in tables (See Tables 2-4) and then used to generate the final codes that were used to supplement the major themes. Each major theme relates to one of the three research questions that were used to answer the overarching question for this study: How do eSupervisors contribute to the learning growth of eInterns?

Before delving into the synopsis of each major theme in this study, the context in which each case took place is described below.

\section{Case-by-Case Context}

\section{Case One}

eSupervisor One's team is responsible for submitting monthly reports concerning an annual project to the government, along with conducting research on numerous facets of the economy. Her eInterns are tasked with researching various economical topics and producing written reports. This is eSupervisor One's second year taking on eInterns from VSFS. During her first year, she only took on three eInterns, but decided to take on six this year. eSupervisor One's preferred method of communication is through email.

eSupervisor One. When asked how she motivates her eInterns, eSupervisor One replied, "I give the interns work to do and it's up to them whether or not they want to do it. It's not my job to make them want to do their job", (Individual interview, March $1^{\text {st }}$, 
2018). eSupervisor One stated that on-the-job training consisted of her giving them instructions on what tasks needed to be completed and then correcting them if they got it wrong, "There is not much training to be had when all you have to do is research something and then write about it" (Individual interview, March $1^{\text {st }}, 2018$ ). She also noted that one of the strengths she noticed in her eInterns is that they are good at following directions - "for the most part" (Individual interview, March $1^{\text {st }}$, 2018). When asked how she tries to enhance their performances, eSupervisor One said that she gives them constant feedback on things that are done well and things that need to be fixed. "I correct them when they need it and let them know they did a good job when they do it" (Individual interview, March $1^{\text {st }}, 2018$ ). eSupervisor One informally evaluates the performance of her eInterns after each assignment is turned in.

As far as what eSupervisor One wants her eInterns to learn from their working experience, she stated, "I want them to learn how to work efficiently and well" (Individual interview, March $1^{\text {st }}$, 2018). Overall, eSupervisor One is pleased with the outcomes of the eInterns she has had so far in VSFS, "I like the program. It is good to have additional assistance when it comes to research. The interns do great work," (Individual interview, March $1^{\text {st }}, 2018$ ). From the varied responses, it seems as if eSupervisor One delivers educational contributions in a directive manner.

Student 1A. Student $1 \mathrm{~A}$ is a current undergraduate sophomore and applied to VSFS because she wanted to "apply the things she was learning is a classroom to real work situations" (Individual interview, March $2^{\text {nd }}, 2018$ ). She did not have any direct goals coming into the program and just did it "purely out of interest" (Individual interview, March $\left.2^{\text {nd }}, 2018\right)$. Student $1 \mathrm{~A}$ has held several jobs over the years and 
considers herself to have a "fairly strong Google search game" (Individual interview, March $2^{\text {nd }}, 2018$ ) because most of the work she is doing now is web-based.

Concerning her experiences on the job, Student 1A stated, "I really enjoy the flexibility that it gives because you can do your work from literally wherever you want, so long as you have internet connection" (Individual interview, March $2^{\text {nd }}, 2018$ ). When asked to describe her working relationship with her eSupervisor, Student 1A described her as being and that she "always gives feedback to the reports we write" (Individual interview, March $\left.2^{\text {nd }}, 2018\right)$. What Student $1 \mathrm{~A}$ does not like is that working without face to face interactions has been an adjustment, "I still prefer a more traditional job experience where you are working physically in the same geographic location as the people around you" (Individual interview, March $2^{\text {nd }}, 2018$ ). When asked how her eSupervisor meet her learning needs, Student 1A went on to say, "We never really discussed it because she knows that we're able to do the work that's assigned" (Individual interview, March $2^{\text {nd }}, 2018$ ).

When asked how her eSupervisor motivated her to do her job, Student 1A replied, “There's not much motivation on her part because...I think, so long as you're interested in it, you'll put in the work" (Individual interview, March 2 ${ }^{\text {nd }}, 2018$ ). eSupervisor One's educational contributions to Student 1A's experience were interpreted as learning the virtue of self-motivated interests. Student 1A's eSupervisor seemed to have a very handsoff approach when it came to generating interest in the projects that were assigned. From this, Student 1A realized that her own interests in the topics are what motivated her to do the work. 
Student 1B. Student 1B is in her last year of undergraduate coursework and applied to VSFS because she was interested in how the U.S. worked with other countries. Student 1B has had various prior work experiences that were somewhat related to her field of interest, which was Political Science. In describing her experiences with VSFS, Student 1B exclaimed, "It has been nice; I like to read and write, but research was definitely something that I wanted to expand on and become stronger in" (Individual interview, March $3^{\text {rd }}$, 2018). Student 1B also mentioned that the best part of the relationship between her and her eSupervisor was that she was very straightforward, "It helps me to do a better job when I know exactly what needs to be done, when it needs to be done by, and what it needs to be done for" (Individual interview, March $3^{\text {rd }}, 2018$ ).

One aspect of the working relationship that Student 1B described as having liked the least was not having the ability to experiment: "When I am learning something new, it helps to have guidelines, but after I get the hang of things it would be nice to try something new...she is very specific about what she wants" (Individual interview, March $3^{\text {rd }}$, 2018). Student $1 \mathrm{~B}$ cited one of her strengths in a virtual environment as "the ability to think outside of the box because there is so much information to be found out there and all at the click of a button" (Individual interview, March $3^{\text {rd }}$, 2018). Student 1B stated that she has learned "the art of efficiency in research" (Individual interview, March $3^{\text {rd }}$, 2018), among other aspects relating to the topics she was researching. eSupervisor One's educational contributions to Student 1B's experience were interpreted as learning the virtue of creativity. While Student 1B acknowledges that having a few guidelines to begin are nice, it seems as if her true passion lies within begin able to come up with her own creative concepts. 
Graduate 1A. Graduate 1A interned with VSFS last year and is currently a graduate student, majoring in International Relations. Her motivation for applying to the internship stemmed from her interest in policy studies and international affairs, "I want to work in a career that focuses on international policy initiatives" (Individual interview, February $\left.21^{\text {st }}, 2018\right)$. This internship was not her first working experience; however, it was her first time working in a virtual environment. When asked to describe a typical work day with VSFS, Graduate 1A detailed research projects that were assigned to her once a month, "The flexibility was great" (Individual interview, February $21^{\text {st }}, 2018$ ), she stated .

Graduate 1A's goals going into VSFS were to gain some experience working with the government and to learn about the economy of another country as well, "I wanted to get some experience working with a government agency and I got it" (Individual interview, February $21^{\text {st }}, 2018$ ). She stated that the only aspect she wanted more out of it was to have some more interaction with her supervisor and maybe the other interns, too: "It was definitely a valuable experience because I got to learn so much about the economy of $[\ldots]$ and was able to do so at my own pace" (Individual interview, February $\left.21^{\text {st }}, 2018\right)$. Graduate $1 \mathrm{~A}$ also mentioned that her time spent at VSFS enabled her to better manage her time with other schoolwork and priorities that she had going on, "I'm in grad school now and there's always so much going on that I wish some of it were virtual, so that I can try to fit all of it in my schedule" (Individual interview, February $\left.21^{\text {st }}, 2018\right)$. The value that Graduate $1 \mathrm{~A}$ seemed to recognize was the value of time management. For her, she would be better able to find the time to do all the 
activities she wanted to do if some of it was flexible enough to manage or held in a virtual environment where she could make her own schedule.

Graduate 1B. Graduate 1B is another female who interned at VSFS during the same time as Graduate 1A. She stated that she was motivated to apply for work there because "working for a foreign nation was intriguing at the time and because this was the only way I would be able to do so" (Individual interview, February $23^{\text {rd }}, 2018$ ). Graduate 1B described her work day at VSFS as being "pretty chill” (Individual interview, February $23^{\text {rd }}, 2018$ ); she mentioned that assignments would get emailed out and she would just have to get them done by the due dates. As for her experiences, Graduate 1B stated, "I liked it. There could have been more interaction, I think, but that could just be the nature of the virtual working environment" (Individual interview, February $\left.23^{\text {rd }}, 2018\right)$. This was Graduate $1 B$ 's first time working in a virtual environment.

Graduate 1B's goals going into the VSFS program were to get some research experience and to add relevant work experience on her resume, "I eventually want to go to graduate school and get an advanced degree in Public Affairs or Economics" (Individual interview, February $23^{\text {rd }}, 2018$ ). Graduate $1 \mathrm{~B}$ is currently an undergraduate Senior, preparing to graduate this year. In regard to what was learned from the internship experience, Graduate 1B stated, "I learned a lot about economics and how to be a better researcher" (Individual interview, February 23 ${ }^{\text {rd }}, 2018$ ). When asked to elaborate on how she became a better researcher, Graduate 1B said that her supervisor was very particular about using credible sources and about how the reports should be organized as well, "If you didn't do it right, she would tell you what needed to be fixed and you would have to resubmit until you got it right...after the first couple of months I finally got the groove of 
things" (Individual interview, February $23^{\text {rd }}, 2018$ ). Graduate 1B described her overall working experience with VSFS as "a great opportunity that helped to set me up for my next working experience" (Individual interview, February $23^{\text {rd }}, 2018$ ). She currently has a fellowship working on various research topics,

I think the research experience that I had with VSFS helped to land me this job. There is a lot more research with this [internship], but I thoroughly enjoy it because I am more familiar with researching and how to find credible sources too" (Individual interview, February $23^{\text {rd }}, 2018$ ).

The value that Graduate 1B seemed to recognize was the value of proficiency.

Graduate 1B had mentioned that her supervisor would send back her reports if they did not meet her standards. Through trial and error, Graduate 1B was able to use the protocols her supervisor required to conduct proper research for her assignments. Her supervisor's actions, in turn, helped Graduate 1B to obtain another working experience where she could directly apply the skills she learned from her prior experience.

\section{Case Two}

eSupervisor Two's team helps with the management of their agency's website, along with the digital and social media channels. His eInterns take on various tasks such as, video editing, graphic design, fact checking, editorial writing, blog tagging, and social media research. During his first year with VSFS, eSupervisor Two took on 30 eInterns. However, he decided to accept all applicants this year, which was about 80, due to unfavorable past experiences. eSupervisor Two described having a number of past eInterns who decided to stop doing work and only a few that he could really depend on. eSupervisor Two stated that he uses a communications software program called Slack; this allows the eInterns and the other members of his team to communicate with each 
other on multiple channels. eSupervisor Two also stated that he uses a task management software program called Trello, to manage all of the tasks needed to be done.

eSupervisor Two. As previously mentioned, eSupervisor Two took on 30 eInterns during his first year. This past year he decided to accept all applicants, which was about 80 , because he was anticipating a decline in eIntern activity levels, "Over time, the activity level of many of the interns kind of falls by the wayside...[Currently] 30 to 40 are actually still doing things that I'm aware of' (Individual interview, February $27^{\text {th }}$, 2018). When asked how he was able to manage so many eInterns, eSupervisor Two stated, "It was a bit of a progression for me coming to the realization that it's kind of hard to actually manage them...I don't control anything...all I can do is ask for them to do things" (Individual interview, February $27^{\text {th }}, 2018$ ).

eSupervisor Two was also adamant about taking a hands-off approach to motivation:

We don't control them. They find things to work on and they work on it... The first year I made attempts to actually get people to do things, like be more active, but towards the end I kind of came to the realization that...I'm just going to get a core group of people that are really good interns I can count on...I have no means by which to control you, so I'm going to just put work out there and if you do it, then thank you very much" (Individual interview, February $27^{\text {th }}, 2018$ ).

When it comes to noticing the strengths of his eInterns, eSupervisor Two mentioned that he does reach out to eInterns who stand out and asks them to take on more of a managerial role: "I've kind of empowered them to go in and direct the other interns to help get their tasks done." eSupervisor Two goes even further to help out those who stand out by passing along pertinent information to them: "If I see a job that has their 
name on in, I definitely forward them that information...those that do work, I'm definitely going to go out of my way to help them out" (Individual interview, February $27^{\text {th }}, 2018$ ). eSupervisor Two has said that he wants his eInterns to learn the ability to, "go out and find something to do...you need to just get in there and do it. Be a self-starter" (Individual interview, February $27^{\text {th }}, 2018$ ). From eSupervisor Two's responses, it seemed as if he delivered educational contributions by using a "hands-off" approach to supportive leadership.

Student 2A. Student 2A was motivated to apply to the VSFS program because she wanted enough experience to "be as competitive as possible" (Individual interview, February $\left.24^{\text {th }}, 2018\right)$ in the graphic design field. She is currently a junior in undergraduate school and wants to pursue a career in the graphic design industry. Student 2A has had prior work experiences, but none in her field of study. She described her experiences with VSFS as being really good. "I like that I'm getting opportunities to exercise my design skills" (Individual interview, February $24^{\text {th }}, 2018$ ). Student $2 \mathrm{~A}$ described the working relationship between her and her supervisor in a positive manner as well, "[My supervisor] is great...I liked that he noticed my commitment to the job and my skills as well...there isn't anything that I don't like about him" (Individual interview, February $\left.24^{\text {th }}, 2018\right)$.

Student 2A exclaimed that her motivation for doing her job stemmed from herself. "I've always been self-driven, so I think that's what motivates me" (Individual interview, February $24^{\text {th }}, 2018$ ). Student $2 \mathrm{~A}$ also stated that her eSupervisor helped with this motivation by escalating her role in the internship to one that reviews some of the tasks that the other eInterns are working on. "It's gotten to the point that I've been asked 
to review other people's designs to see if there are any improvements that can be made" (Individual interview, February $24^{\text {th }}, 2018$ ).

When asked about what she has learned so far in the program, Student 2A stated, "I learned that hard work pays off" (Individual interview, February $\left.24^{\text {th }}, 2018\right)$. She went on to say, "I think I just want to know how I can keep applying myself to be better the next time around" (Individual interview, February $\left.24^{\text {th }}, 2018\right)$. eSupervisor Two's educational contributions to Student 2A's internship experience were interpreted as learning the value of empowerment.

Student 2B. Student 2B is an undergraduate Senior who exclaimed that working with the specific VSFS agency that he was in, was not his first or second choice, but "is glad [he] decided to accept [his eSupervisor's] invitation to join the team" (Individual interview, February $3^{\text {rd }}, 2018$ ). When describing his working experiences, Student $2 \mathrm{~B}$ said, "I am so grateful for this opportunity. We pick up tasks from time to time and just work on them until they are complete" (Individual interview, February $3^{\text {rd }}, 2018$ ). He also said, 'I'll admit that my design skills were pretty basic when I started, but [my eSupervisor] sets us up with lots of resources and training videos to help us out" (Individual interview, February $3^{\text {rd }}, 2018$ ). Student 2B felt much gratitude for being hired and went on to say, "I don't want to let [my eSupervisor] down. He gave me a chance, so now I have to prove that I was worth it" (Individual interview, February $3^{\text {rd }}$, 2018).

When asked about his strengths in a virtual environment, Student 2B stated, "I’ve always been good with tech, so I guess that's a strength. And I'm really good at learning new tech, too" (Individual interview, February $3^{\text {rd }}, 2018$ ). In regard to what has been learned so far, Student 2B said, "I learned how to be a better designer" (Individual 
interview, February $3^{\text {rd }}$, 2018). He also said, "I just want to keep learning how to be a better designer" (Individual interview, February $3^{\text {rd }}, 2018$ ). eSupervisor Two's educational contributions to Student 2B's learning experience were interpreted as learning the value of growth.

Graduate 2A. Graduate 2A worked in the VSFS program last year and is currently working at an embassy. She was motivated to apply for the VSFS internship because she needed an internship in her field of interest, communications and digital media. Eventually, Graduate 2A wants to go to graduate school and continue within the realm of communications. "I'm kind of keeping it open as I continue with my new job and know what specific things I like and what things I don't like...whether that's speech writing or creating digital content or being a press officer, I'm still trying to narrow that down" (Individual interview, February $27^{\text {th }}, 2018$ ).

While working with VSFS, Graduate 2A had several other working responsibilities. She was a social media administrator, an honors writing fellow, a writing tutor, and was involved in another fellowship program all while taking classes in her senior year of undergraduate school. Graduate 2A described her experiences with VSFS as being "great" because her eSupervisor acknowledged the work she did compared to other positions that she had: "I was really feeling fulfilled, and like I was doing a good job and being appreciated for doing a good job...because my supervisor would every so often call and say, 'you're doing great work"' (Individual interview, February $27^{\text {th }}$, 2018). Graduate 2 A went on to say, "That really shaped my experience...because I was able to compare and realize that this is what was motivating, is having a good supervisor. Sometimes that makes all the difference" (Individual interview, February $27^{\text {th }}, 2018$ ). 
Graduate 2A's goals going into VSFS were to make sure she could manage her time well, learn how to work in a virtual space, and gain more knowledge on digital media and the communications aspect of the government, "All my goals were met and beyond. So, it was a great experience for me" (Individual interview, February $27^{\text {th }}, 2018$ ). While her goals were met, Graduate 2A also stated, "My main takeaway was that it really matters who you work with, and your supervisor can make all the difference in how you feel about the quality of the work that you're putting out" (Individual interview, February $\left.27^{\text {th }}, 2018\right)$.

As for how she is applying everything that she has learned, Graduate 2A said, "In the coming years...I will make sure that whatever I do in the future is both actual content I like and that I am working with a good group of people, with good leadership and good communication skills, because that's just as important to me" (Individual interview, February $\left.27^{\text {th }}, 2018\right)$. It seemed as if that the value that Graduate $2 \mathrm{~A}$ recognized with this internship was the value of feeling appreciated.

Graduate 2B. Graduate 2B is currently an undergraduate senior who interned with VSFS last year and chose to intern with the same program again this year. When asked how he is applying the skills that he's learned from last year, Graduate 2B stated, "I have more of a managerial role now, which is kind of cool because it shows that my supervisor appreciates the work that I did last year" (Individual interview, February $19^{\text {th }}$, 2018).

Graduate 2B's original goals when going into the program last year were to get more experience in graphic design and to do something that did not conflict with his school schedule. He returned this year because, "It was too great of an opportunity to 
pass up" (Individual interview, February 19 $9^{\text {th }}, 2018$ ). Graduate 2B said that his goals were achieved last year because, "I got to decide how much experience I could get by being able to choose the projects that I wanted" (Individual interview, February $19^{\text {th }}$, 2018). Graduate 2B also said that he learned, "When you see an opportunity, you have to go for it" (Individual interview, February 19 ${ }^{\text {th }}, 2018$ ).

When asked what else would he want to learn, Graduate 2B simply stated, "In graphic design, there is always more to learn. That is why I decided to do the internship again this year" (Individual interview, February $27^{\text {th }}, 2018$ ). In this case, it seemed that the value that Graduate 2B recognized with his internship was the value of opportunity.

\section{Case Three}

This is eSupervisor Three's third time taking on eInterns from VSFS and he states that he has evolved his management tactics with each year (Individual interview, February $\left.26^{\text {th }}, 2018\right)$. eSupervisor Three currently works with four eInterns. In previous years, he has only had two at a time. eSupervisor Three works on a team that manages most of their agency's digital instructional projects. The team works on projects that are designed to provide training experiences for the users. He put together a multi-faceted team of eInterns to help him initiate various projects. eSupervisor Three likes to experiment with different communication methods and usually settles with the one that is

most conducive for the team. This year, the team's preferred method of communication is an application called WhatsApp (2018).

eSupervisor Three. eSupervisor Three actually had to recruit his eInterns this year because he did not have any qualified applicants after the application deadline had passed. The eInterns that agreed to work with him were not picked for their first choices 
and had turned down all other offers. "What I did to motivate them to accept the position was to inform them that it was very malleable. They could tailor it to their own skills and to what they're trying to learn more about" (Individual interview, February $26^{\text {th }}, 2018$ ). eSupervisor Three also placed an emphasis on acknowledging their work, "To motivate them, I really gave them creative ownership" (Individual interview, February $26^{\text {th }}, 2018$ ).

When asked to describe how he facilitated on-the-job training, eSupervisor Three stated, "The training is the other way around. They're training me on new tools, new concepts, and new approaches that are used in the schools they go to" (Individual interview, February $\left.26^{\text {th }}, 2018\right)$. eSupervisor Three also provides interns with suggested links and resources which they then, "digest in their own time and in their own way" (Individual interview, February $26^{\text {th }}, 2018$ ). There are also many discussions that take place where they can clarify the project guidelines. eSupervisor Three stated, "A lot of it is case-by-case training, but...it's basically learn by doing, not listening" (Individual interview, February $\left.26^{\text {th }}, 2018\right)$.

eSupervisor Three also tries to enhance his eInterns' performances by including them in the decision making, "I've tried to enhance them by giving them the authority and looking to them as Junior Consultants" (Individual interview, February $26^{\text {th }}, 2018$ ). From his responses, it seemed as if eSupervisor Three delivered educational contributions by using a supportive and participative approach.

Student 3A. Student 3A is an undergraduate junior who is currently studying abroad in India. As part of her degree program, she has been conducting a remote internship for the past three years and is presently conducting it in conjunction with the VSFS internship. Student 3A's motivation for working with VSFS was to, "see what the 
process [working with the government] is and see what it's like to work on a project that is fulfilling in a way that is giving back to society" (Individual interview, March $2^{\text {nd }}$, 2018).

Student 3A described her eSupervisor as someone who, "cares a lot and wants to find a way to make this experience fulfilling to us as well as useful and helpful to him" (Individual interview, March $2^{\text {nd }}, 2018$ ). eSupervisor Three was also described as “incredibly approachable and very communicative" (Individual interview, March $2^{\text {nd }}$, 2018). Student 3A stated that her eSupervisor took a hands-off approach to her learning needs, "He works with us really closely when we need help, but likes us trying things our own...I think that's one of the best ways to learn" (Individual interview, March $2^{\text {nd }}$, 2018).

In listing her strengths working in a virtual environment, Student $3 \mathrm{~A}$ referred to her enrollment at an educational technology university by exclaiming, "I know how to communicate and build relationships at a distance because I do it so often" (Individual interview, March $2^{\text {nd }}, 2018$ ). Interestingly, Student 3A stated later on that one of the weaknesses she had working in a virtual environment was that, "because I'm so far away, my time zones don't work well...it's tough to get to know them on a better level" (Individual interview, March $2^{\text {nd }}, 2018$ ). Student 3 A went on to say that she's learned that she "would rather not have a remote job full-time, unless I have a very strong community." eSupervisor Three and the other eInterns are all located in the U.S. Even though Student 3A is accustomed to working and taking classes remotely, this is the first time she has ever worked with other people who were so far geographically separated. This finding was an anomaly as Student 3A was the only student interviewed who was 
located on a different continent. When asked how her eSupervisor has addressed these concerns, Student 3A admitted that she has not brought it up to him, "I guess, I didn't even realize it until now that it was something I was feeling" (Individual interview, March $\left.2^{\text {nd }}, 2018\right)$. eSupervisor Three's educational contributions to Student 3A's virtual internship experience were interpreted as realizing the need for a sense of community.

Student 3B. Student 3B is an undergraduate junior who chose to apply for the VSFS program because he wanted to get some experience working with data. "I'm interested in a lot of things, so doing this internship is a way to get my feet wet without really jumping in the water" (Individual interview, February $3^{\text {rd }}, 2018$ ). Student 3B currently works with Student 3A and two other eInterns in their department. "It's really great being part of a team that works on so many different projects that cater to our specific skill sets" (Individual interview, February $3^{\text {rd }}, 2018$ ).

Student 3B described his experiences with VSFS as "extremely worth it" and "something that I will definitely look into again next year to try out a different skill" (Individual interview, February $3^{\text {rd }}, 2018$ ). When asked he's learned so far in the program, Student 3B replied, "I've learned a lot about how to work professionally with others virtually, but mostly how to think creatively about working with data sets" (Individual interview, February $3^{\text {rd }}, 2018$ ). While Student 3B is the only eIntern on his team with strong data analysis skills, the other eInterns have varied skills that have been useful supplements to some of their projects. eSupervisor Three's educational contributions to Student 3B's learning experience were interpreted as learning the value of creative thinking. 
Graduate 3A. Graduate 3A is a current graduate student, who works as a marketing coordinator in a secondary school setting. She chose to work with the VSFS program last year because conducting an internship was a requirement for her program and a virtual internship allowed for the flexibility she needed at the time. This was not her first working experience as she has previously worked on campus with several different departments. Aside from the VSFS internship, she also held two other part-time jobs at the time.

Graduate 3A worked with another eIntern during her time with VSFS and their workload was dependent on how they decided to split up the projects. She exclaimed, "It was really nice because I had opportunities to do presentation slides, do GIFs, and create things I wouldn't necessarily do" (Individual interview, March $1^{\text {st }}$, 2018). Graduate 3A's main goal going into the internship was to make sure she, "did a good job and learn something new" (Individual interview, March $1^{\text {st }}, 2018$ ). Graduate $3 \mathrm{~A}$ also stated that working remotely made things easier in her transition to her work in graduate school.

When sharing her overall experience with VSFS, Graduate 3A stated,

I think that's the thing that's kind of really unique about it...you're working remotely and working with your supervisor. Unless you're in the area you're probably not going to meet the person but I think it's cool to have that kind of mentorship and have the opportunity to kind of learn and grow and do different projects you probably wouldn't be exposed to otherwise" (Individual interview, March $1^{\text {st }}, 2018$ ).

Later on, Graduate 3A shared that, "It's really a self-starter situation and I'm responsible for what I'm giving. It really gave me the opportunity to be responsible and accountable for what I signed up for" (Individual interview, March 1 ${ }^{\text {st }}, 2018$ ). From her responses, it 
would seem that that Graduate $3 \mathrm{~A}$ recognized the values of accountability and opportunity with this internship.

Graduate 3B. Graduate 3B is currently an undergraduate senior doing freelance graphic design work, while working a part-time job. She decided to join the VSFS program because she wanted to see if a career in graphic design was a path she wanted to take: "I like designing on my own time, and I wanted to see if it was something I could possibly do full-time" (Individual interview, February $7^{\text {th }}, 2018$ ). She worked with eSupervisor Three at the same time as Graduate $3 \mathrm{~A}$.

Graduate 3B described her working experiences as, "Pretty cool, because this was my first time working in design in a professional environment" (Individual interview, February $\left.7^{\text {th }}, 2018\right)$. She also described eSupervisor Three as, "someone who really wanted to make sure you were prepared to do what you were doing before you did it" (Individual interview, February $7^{\text {th }}, 2018$ ). Graduate 3B was asked to further detail this comment and said that before each project, her eSupervisor would always send out an email with instructions, give them time to ask clarifying questions, and would also have periodic check-in points to see how the work was going: "We could discuss over the phone or through email; it was really up to us and what we felt comfortable with" (Individual interview, February $\left.7^{\text {th }}, 2018\right)$.

When asked what she found to be the most valuable, Graduate 3B answered, "The ability to work remotely" (Individual interview, February $7^{\text {th }}, 2018$ ). Graduate 3B stated that she has been searching for a full-time graphic design or digital media-related position that will allow her to work remotely because she plans on going to graduate school and will need the flexibility and the funds to do both: "With the programs that I have been 
looking at, their schedules are pretty hectic and I was worried about being able to get a job to pay my bills, but now I know it's possible" (Individual interview, February $7^{\text {th }}$, 2018). From the responses, it seemed that Graduate 3B recognized her internship provided her the value of flexibility.

\section{Case Four}

eSupervisor Four works on foreign initiatives that include educating the local populations on diplomatic efforts with the U.S. eSupervisor Four currently supervises 10 eInterns and they are tasked with researching various topics and creating presentations to display their findings. eSupervisor Four communicates with her eInterns individually through email, but also has a Google Doc spreadsheet that everyone uses to log in their progress on assignments.

eSupervisor Four. eSupervisor Four has worked with VSFS the past couple of

years and has thoroughly enjoyed its presence. "The interns are great. They have been so helpful in helping our department achieve its goals" (Individual interview, February $3^{\text {rd }}$, 2018).

When asked about her motivation tactics for her eInterns, eSupervisor Four replied, "I think that letting them have ownership over their projects gives them a sense of duty that can't be taken away" (Individual interview, February $3^{\text {rd }}, 2018$ ). For eSupervisor Four, letting her eInterns take control over their projects serves many purposes, "I mean, we take on eInterns so that they can help make our jobs easier. So, by leaving them to their own merits, I can focus on more important things and they can learn to come up with ideas of their own...become critical thinkers" (Individual interview, February $\left.3^{\text {rd }}, 2018\right)$. 
Regarding the strengths and weaknesses of her eInterns, eSupervisor Four stated, "Some of them just take off and come up with really great ideas, and others just need a little more guidance and time" (Individual interview, February $3^{\text {rd }}, 2018$ ). She said for those that need more guidance, she provides them with examples of what has been done before, "That usually does the trick" (Individual interview, February $3^{\text {rd }}$, 2018). eSupervisor Four stated, "I want them to learn how to be independent and how to take control of their skills and figure out how to use them to the best of their abilities" (Individual interview, February $3^{\text {rd }}, 2018$ ). From her responses, it seemed as if eSupervisor Four delivered educational contributions by using a "hands-off" approach.

Student 4A. Student 4A is an undergraduate senior who likes being creative and innovative. She mentioned that this was one of the reasons why she likes this internship, "because it wasn't so structured and gave me the chance to be innovative" (Individual interview, February $\left.28^{\text {th }}, 2018\right)$. Student 4 A was originally motivated to apply for the VSFS internship because she thought it would be a good opportunity to improve her speaking skills as well as build on her resume since she is an International Studies major. The VSFS internship is not her first working experience as she has had prior varied working experiences. Student 4A is currently working a part time job while taking on this internship.

When asked to describe her experiences with VSFS so far, Student 4A exclaimed that they were, "rewarding, but challenging because of the time difference, not physically being there, and some miscommunications" (Individual interview, February $28^{\text {th }}, 2018$ ). Student 4A was asked to elaborate on the miscommunications, but she would not go into any clear details. Student 4A also described the relationship between her and her 
eSupervisor as being "very basic and minimal" (Individual interview, February $28^{\text {th }}$, 2018). She stated that the one thing she would have changed is that, "I would have liked more of a mentorship opportunity...that would have been beneficial" (Individual interview, February $\left.28^{\text {th }}, 2018\right)$.

In regard to what more she wanted to learn from the internship, Student 4A said, "I would like to reach out to learn more about my supervisor and try to build a relationship and become more acquainted...I don't just want to only put it on the supervisor because I think that I should have taken better initiative to do that" (Individual interview, February $28^{\text {th }}, 2018$ ). Student 4 A initially did not know what to expect when coming into this program but states that is has been a positive experience overall, "Anything that I learned is of value to me" (Individual interview, February $28^{\text {th }}, 2018$ ). eSupervisor Four's educational contributions to Student 4A's virtual internship experience were interpreted as learning the need to take initiative.

Student 4B. Student 4B is an undergraduate junior majoring in Political Science with an interest in "the education side of things" (Individual interview, February $27^{\text {th }}$, 2018). Student 4B applied to work for VSFS because of her interest in other cultures, "I really value cross-cultural experiences" (Individual interview, February $27^{\text {th }}, 2018$ ). Aside from working with VSFS, Student 4B is also working a part-time job on campus. When describing her experiences with the program, Student 4B mentioned that there has been very little contact and virtually no guidance. However, she later exclaimed that the flexibility and independence was one of the best parts of her relation with her eSupervisor, "Having this independent nature to it has been really valuable" (Individual interview, February $27^{\text {th }}, 2018$ ). When clarifying her comments, Student $4 B$ revealed that 
what she did not like in the beginning of the program ended up being what she liked later on. Her motivation for doing her job is, "being able to connect with people that I would not have been able to connect with in my daily life" (Individual interview, February $27^{\text {th }}$, 2018). eSupervisor Four's educational contributions to Student 4B's learning experience were interpreted as recognizing the value of autonomy.

Graduate 4A. Graduate 4A recently graduated from undergraduate school last year and is currently working in another internship program. In her time spent with VSFS last year, Graduate 4A stated that it "showed me a different way of life that I wasn't aware of" (Individual interview, February $\left.16^{\text {th }}, 2018\right)$. In this context, she was referring to the cultural topics she researched and presented on. Graduate 4A initially applied to VSFS because she knew someone else who did the program before her and it was highly recommended.

In describing her learning experience, Graduate 4A explained that she already had an idea of what to expect due to prior information from a friend. She said, "My supervisor did not contact us often, but that was fine with me... I have a friend who did something similar to this before me, so I always had someone that I could turn to right away" (Individual interview, February $16^{\text {th }}, 2018$ ). Graduate $4 \mathrm{~A}$ also stated that the resources she was given at the beginning of the program were very helpful, "I liked the whole 'self-guided' format of working, I just wish there were more jobs like this" (Individual interview, February $16^{\text {th }}, 2018$ ). While she was working with VSFS, Graduate 4A was taking a heavy load of classes which took up a lot of her time. "I honestly just did it purely out of interest. Since it was promoted as being flexible with your schedule, I thought...why not?” (Individual interview, February $16^{\text {th }}, 2018$ ). From 
her responses, it seemed that the value that Graduate 3B recognized with this internship was the value of self-sufficiency.

Graduate 4B. Graduate 4B is a current undergraduate senior who applied to VSFS because of his interest in being a Foreign Services Officer. Graduate 4B stated, "I applied because it was new and exciting and offered so many opportunities to be involved in foreign affairs" (Individual interview, February $3^{\text {rd }}, 2018$ ). Graduate 4B also serves in the U.S. military and decided to take on another virtual internship so that he can get more experience outside of the military because. "I love the fact that I can do it pretty much anytime because my schedule is just pretty crazy" (Individual interview, February $3^{\text {rd }}$, 2018).

When asked to describe his work experiences, Graduate 4B stated, "My only complaint is that there was little to no guidance...there was no real sense of what they wanted. At the end of the day it's understandable why they kept it open" (Individual interview, February $3^{\text {rd }}, 2018$ ). Graduate 4B also mentioned that the fact that her virtual internship been self-regulated has been nice. His eSupervisor has also "always given positive feedback" (Individual interview, February $3^{\text {rd }}, 2018$ ), so he thought that was nice, as well. In regard to what Graduate 4B learned from the experience, he replied, "I learned how to be flexible with my time" (Individual interview, February $3^{\text {rd }}, 2018$ ). From his responses, it seemed that the value that Graduate 4B also recognized with this internship was the value of flexibility. 


\section{Research Questions}

The initial codes, expanded codes, and final codes generated for each major theme in this study are listed in Tables 2-4. The analyses for each research question below discuss the final codes that were interpreted for each major theme.

\section{RQ1: eSupervisory Style}

In order to answer the research question, "How do eSupervisors describe their delivery of educational contributions that promote learning in a virtual internship environment?", an interview protocol (Appendix B) was administered to four eSupervisors in this study. Background questions were asked during the interviews to gain an idea of where the eSupervisors were from, the environment that they worked in, their typical job responsibilities, and a sense of their work ethic. The interview questions were specifically selected to try and obtain data that would orient the eSupervisor's leadership style to those styles that were presented in the literature review. The interviews were also structured this way to get the eSupervisors to begin thinking about the reality of their work style and how supervising their eInterns fit within that reality.

Responsibility. Responsibility was interpreted as a final code under this theme as it seemed that each eSupervisor thought their eInterns should be motivated by having a sense of responsibility or ownership over their work. There was a slight dichotomy in the methods that were initiated by the eSupervisors, where some would be direct in their intentions and others worked more indirectly; however, the overall intent of having motivated eInterns remained the same. For example, eSupervisor One worked more indirectly because she did not believe that it was her job to motivate her eInterns: "I give the interns work to do and it's up to them whether or not they want to do it. It's not my 
job to make them want to do their job" (Individual Interview, March $1^{\text {st }}, 2018$ ).

eSupervisor Four worked more directly, "I think that [giving] them ownership over their projects gives them a sense of duty that can't be taken away" (Individual Interview, February $3^{\text {rd }}$, 2018).

Responsibility was also interpreted as a learning goal that the eSupervisors wanted their eInterns to achieve. Specifically, the eSupervisors wanted their eInterns to be responsible for their actions: "I want them to learn how to work efficiently and well" (eSupervisor One, Individual Interview, March 1" 2 2018); “I want my eInterns to learn the ability to go out and find something to do...you need to just get in there and do it. Be a self-starter" (eSupervisor Two, Individual Interview, February 27 $7^{\text {th }}, 2018$ ); "I want my interns to learn how to make mistakes. I want them to learn that they don't have to be perfectionists" (eSupervisor Three, Individual Interview, February 26 $6^{\text {th }}, 2018$ ); “I want them to learn how to be independent and how to take control of their skills and figure out how to use them to the best of their abilities" (eSupervisor Four, Individual Interview, February $3^{\text {rd }}$, 2018).

Empowerment. Empowerment was coded separately from responsibility because there were some eSupervisors who seemed to specifically want to empower their eInterns, outside of just giving them responsibility. eSupervisor Two even went so far as to "promote" those eInterns who stood out among the rest: "I've kind of empowered them to go in and direct the other interns to help get their tasks done" (eSupervisor Two, Individual Interview, February $27^{\text {th }}, 2018$ ). While, eSupervisor Three took a different approach: 
"What I did to motivate them to accept the position was to inform them that it was very malleable. They could tailor it to their own skills and to what they're trying to learn more about...to motivate them, I really gave them creative ownership" (Individual Interview, February 26 ${ }^{\text {th }}, 2018$ ).

Resources. Interestingly, each eSupervisor conducted on-the-job training differently. This could be attributed to the specific job duties that their eInterns had to carry out. However differently each eSupervisor may have conducted training, each one gave their eInterns a resource in their own right. For example, eSupervisor One gave her eInterns specific instructions to be followed when writing up reports; her eInterns were only conducting research. Likewise, eSupervisor Four sent her eInterns information at the beginning of the program on what needed to be done and the purpose for the projects. She also gave her eInterns some "tips to succeed" (Student 4B, Individual Interview, February $\left.27^{\text {th }}, 2018\right)$ that were passed on by her previous eInterns; her eInterns were tasked with researching topics and creating presentations to follow. eSupervisor Two created a variety of training videos, writing style guides, and sends out suggestions for training resources too. Thus far, eSupervisor Three seemed to be the only supervisor with an overall action plan:

On the job training is me sending them either to a website or resource, a video or some sort of explainer content that explains a concept that I want to work on. So, I basically provide them suggested links and resources that they basically digest in their own time and in their own way. Then, they have the open opportunity to ask questions and clarifications through one-on-one discussions" (Individual Interview, February $26^{\text {th }}, 2018$ ).

Regular Feedback. Each of the projects or tasks that the eInterns had to work on had different deadlines that rotated on a weekly, monthly, or varied basis. When assignments were turned in, eSupervisors had an opportunity to evaluate the work and offer feedback. eSupervisor Two had his eInterns submit weekly reports to keep track of 
the work they did and so they could use as a reference to create bullet points for their resumes. eSupervisor One and eSupervisor Four offered feedback as needed and eSupervisor Three had a different method of evaluating his eInterns:

Not only did they do it on time, but did they do a good job, and could have this been done without a virtual intern? Those three factors come into play with how I evaluate them (Individual Interview, February $26^{\text {th }}, 2018$ ).

To recap, Research Question 1 was, "How do eSupervisors describe their delivery of educational contributions that promote learning in a virtual internship environment?" There were two sub-questions that were considered in answering this question: "What do eSupervisors want their eInterns to learn during their virtual internships?" and "How are eSupervisors helping eInterns accomplish their learning goals?” Each of the final codesempowerment, responsibility, resources, and regular feedback — were categorized under the major theme of "supervisory style" because a combination of methods used for each was seen as integral in answering the research question. While the eSupervisors used a combination of tactics to promote learning, each one seemed to lean either towards a more definitive approach of leadership or a combination: directive, participative, supportive, or achievement oriented.

In answering the research question, eSupervisors use a variety of tactics that are conducive to their working environments as well as the tasks at hand. They take into consideration the skills of the eInterns that work for them and try to incorporate ways in which the eInterns will grow, according to what each eSupervisor thinks is an important aspect to learn in the workforce. 


\section{RQ2: eSupervisory Relationship}

In order to answer the research question, "How do eInterns interpret the educational contributions that they receive from their eSupervisors into learning experiences?", an interview protocol (Appendix C) was administered to eight students in this study. Background questions were asked during the interviews to get an idea of where the students were from, their typical job responsibilities, and if they had other responsibilities outside of school and the internship. The interviews were structured this way to get an idea of the student persona that decides to take on a virtual internship. Interestingly, all of the students had other working responsibilities outside of the VSFS program, and many of them had multiple educational or research activities that they were involved in as well. Another interesting aspect is that many of the students mentioned that participating in a virtual internship was the only way they could get experience in their fields of interest because they were located in areas that did not offer any on-site opportunities.

Communication. In terms of the working relationship between eInterns and their eSupervisors, "communication" was interpreted as a final code because it was seen as a benefit to some eInterns and a missed opportunity for others. For example, one eIntern stated, "The best part of the relationship between me and my eSupervisor is that she is very straightforward" (Student 1B, Individual Interview, March $3^{\text {rd }}$, 2018). Another eIntern discussed what he found to be beneficial, "I learned how to structure emails to

communicate professionally" (Student 3B, Individual Interview, February $3^{\text {rd }}$, 2018). Additionally, an eIntern described her eSupervisor as "Incredibly approachable and very communicative" (Student 3A, Individual Interview, March $2^{\text {nd }}, 2018$ ). 
Alternatively, other eInterns felt much differently. One described her relationship as "very basic and minimal" (Student $4 \mathrm{~A}$, Individual Interview, February $28^{\text {th }}, 2018$ ) and went on to say, "I would have liked more of a mentorship opportunity...that would have been beneficial." Student 4B mentioned as well that there had been "very little contact and virtually no guidance" (Individual Interview, February $27^{\text {th }}, 2018$ ) between her and her eSupervisor.

Self-Initiated. Each of the students interviewed had a goal coming into the VSFS program that was based on becoming proficient in their skills or just gaining new experience. Whichever the case, they were not being coerced into participating or felt that this was an experience that they needed to have. It was something they wanted to do and were coming in wanting to learn something new. One eIntern stated, "I did not have any direct goals coming into the program and just did it purely out of interest" (Student 1A, Individual Interview, March $\left.2^{\text {nd }}, 2018\right)$. Another eIntern valued being given the opportunity to learn on her own, "[My eSupervisor] works with us really closely when we need help, but likes us trying things our own...I think that's one of the best ways to learn" (Student 3A, Individual Interview, March $2^{\text {nd }}, 2018$ ).

Some eInterns even liked learning that the virtual working environment opened up a whole new realm of possibilities, "It's really exciting to see how I can make a difference in government affairs where location isn't a restriction to what I can do...It's a challenge to work virtually, but rewarding when it works" (Student 3B, Individual Interview, February $\left.3^{\text {rd }}, 2018\right)$; “Shows you a different way of life that you're not aware of. It also allows you to connect with people you may meet later on" (Student 4B, Individual Interview, February $27^{\text {th }}, 2018$ ). 
One eIntern came to the realization that maybe she should be the one to initiate contact with her eSupervisor if she wanted to get more out of her learning experience:

I would like to reach out to learn more about my supervisor and try to build a relationship and become more acquainted...That's not even something that registered to me that I can do that because it is a virtual internship...I don't just want to only put it on the supervisor because I think that I should have taken better initiative to do that (Student 4A, Individual Interview, February $28^{\text {th }}$, 2018).

Motivation. Regarding motivation, all of the eInterns, in some way, felt that they were the ones who motivated themselves more than their eSupervisors. For example, one eIntern described her eSupervisory experience, saying “There's not much motivation on her part because...I think, so long as you're interested in it, you'll put in the work" (Student 1A, Individual Interview, March $2^{\text {nd }}$, 2018). Another eIntern said, "I've always been self-driven, so I think that's what motivates me" (Student 2A, Individual Interview, February $\left.24^{\text {th }}, 2018\right)$. One of the eInterns who did not make the first or second round picks, but was picked up by his eSupervisor later on in the semester stated, “I don't want to let [my eSupervisor] down. He gave me a chance, so now I have to prove that I was worth it" (Student 2B, Individual Interview, February $3^{\text {rd }}$, 2018).

Facilitation of learning. The facilitation of learning was self-initiated as well. The eInterns had the resources available to them and it was up to them whether they wanted to take advantage of them or not. One eIntern eloquently pointed out, "Because a lot of it is web-based, I have fairly strong Google search game", (Student 1A, Individual Interview, March $\left.2^{\text {nd }}, 2018\right)$. Another eIntern stated, “I've learned that hard work pays off”, (Student 2A, Individual Interview, February $24^{\text {th }}, 2018$ ). 
To recap, Research Question 2 was "How do eInterns interpret the educational contributions that they receive from their eSupervisors into learning experiences?" There were two sub-questions that were considered in answering this question: "What do current eInterns wish to learn during their virtual internships?" and "What do current eInterns perceive they are learning during their virtual internships?” The final codescommunication and self-initiated —were thought to be the best at representing the major theme "supervisory relationship" because the basis of any relationship begins with communication and requires someone to "take the first step." In the case of virtual internships, many of the eInterns realized that taking the initiative to apply to their programs also meant continuing to take initiative when it came to becoming proficient and reaching their goals. Some of the eInterns appreciated the benefit of working with an eSupervisor, such as eSupervisor Three, who communicated effectively with them, while others wanted more out of their relationships with their eSupervisors, such as eSupervisor Four.

In answering the research question, these eInterns have seemed to learn that being self-driven is the key to achieving their goals. While there were some eSupervisors, such as eSupervisor Two and eSupervisor Three, who were more supportive than others, there was no "hand-holding" or "cracking of the whip" in this context. The eInterns have learned that it was up to them whether or not they wanted to do the work. The eInterns also learned that effective communication is a necessary component in working relationships and that there are many possibilities available to them in a virtual working environment. 


\section{RQ3: Learning Outcomes}

In order to answer the research question, "How do virtual internships deliver educational contributions that add value to an eIntern's post-internship experience?", an interview protocol (Appendix D) was administered to eight graduates in this study. Background questions were asked during the interviews to get an idea of where the graduates were from, where they worked when they were with VSFS, and what was their motivation for applying there. The interviews were structured this way to be persistent with obtaining an idea of the student persona that decides to take on a virtual internship.

Subject-Matter. If critiques have any inquiries as to whether virtual interns can learn job-related topics or not, these graduates, along with many students have answered them: "I learned a lot about economics and how to be a better researcher" (Graduate 1B, Individual Interview, February $\left.23^{\text {rd }}, 2018\right)$; “Learning in the field, especially as an intern or student is really important" (Graduate 2A, Individual Interview, February $28^{\text {th }}, 2018$ ).

Self-Management. Aside from subject-matter, self-management was a huge area of learning outcomes from the participants in the VSFS program. For example, one graduate discussed how she learned how to hold herself accountable in the work that she does:

The biggest thing I learned was really more of a management thing I think. The whole delivering the daily reports from my side that really encouraged me to actually think of things in terms of actual like metrics and what I was getting accomplished and also... how I could hold myself accountable (Graduate 2B, Individual Interview, February 19 ${ }^{\text {th }}, 2018$ ).

Another graduate felt similarly, "It's really a self-starter situation and I'm responsible for what I'm giving. It really gave me the opportunity to be responsible and accountable for what I signed up for" (Graduate 3A, Individual Interview, March $1^{\text {st }}$, 
2018). Learning how to manage your time is also a huge part of self-management, "I learned how to be flexible with my time", (Graduate 4B, Individual Interview, February $3^{\text {rd }}$, 2018).

Accomplished. Most of the graduates who interned with VSFS in prior years did so out of pure interest or because they wanted to gain some experience in their chosen fields of study. Many of them left with a sense of accomplishment for various reasons. For example, one graduate mentioned the impact of the internship on her next experience saying, "It was a great opportunity that helped to set me up for my next working experience" (Graduate 1B, Individual Interview, February $\left.23^{\text {rd }}, 2018\right)$. Another graduate valued the experience she had with her eSupervisor: "I was really feeling fulfilled and like I was doing a good job and being appreciated for doing a good job...because my supervisor would every so often call and say, 'you're doing great work"' (Graduate 2A, Individual Interview, February $\left.28^{\text {th }}, 2018\right)$.

Other graduates were proud of the work that they accomplished: "I had to put in all this effort and I had done my best to throughout that internship and in the end it did pay off" (Graduate 2B, Individual Interview, February $19^{\text {th }}$, 2018). "It was really cool to see the projects that you did have an impact" (Graduate 3A, Individual Interview, March $\left.1^{\text {st }}, 2018\right)$.

To recap, Research Question 3 is, "How do virtual internships deliver educational contributions that add value to an eIntern's post-internship experience?" There were two sub-questions that were considered in answering this question: "How do past eInterns describe what they learned during their virtual internships?" and "How do past eInterns describe how they are applying the skills they have learned from their virtual internship 
experience?" The final codes: subject-matter, self-management, and accomplished, were generated as supplements to the major theme of "learning outcomes." Most of the eInterns described their experience with VSFS as one that supplemented the knowledge and experience they were able to use for their post-internship experiences. Some of the eInterns went on to get jobs, some participated in another internship, others started graduate school and a few continued on in their undergraduate studies. Being able to master the art of managing your time, holding yourself accountable, and seeing the benefits of a flexible work environment was also a major learning experience for these eInterns. Finally, knowing that the work they completed had some sort of impact or just knowing that they were able to accomplish their goals left the eInterns with a sense of satisfaction and fulfillment. That experience empowered them to continue on accomplishing more goals.

In answering the research question, virtual internships deliver a variety of educational contributions, however, these contributions are perceived to be valuable to an eIntern depending on where and how they choose to apply the skills they have learned.

\section{Summary}

The results from the qualitative multi-case study were presented and analyzed in this chapter. This study analyzed the overall intent of supervisors when it came to supervising virtual interns. A discussion of the theoretical framework in relation to the findings is discussed in Chapter 5. 


\section{CHAPTER FIVE: DISCUSSION}

The problem explored by this study was the lack of research on how the working relationships between eSupervisors and their eInterns generate learning outcomes that contribute to society. This study explored certain leadership methods that eSupervisors were utilizing with eInterns through the theoretical lenses of House's (1971) path-goal theory of leadership and the effectiveness of these methods on learning outcomes. The overarching research question that guided this study was: How do eSupervisors contribute to the learning growth of eInterns? A qualitative multi-case study was conducted on a population of eSupervisors, current eInterns (students), and past eInterns (graduates) that were associated with the Virtual Student Foreign Service (VSFS)

program. VSFS is a program aimed at exposing students to working opportunities in the government.

Educational institutions and other educational programming efforts are tasked with ensuring that their contributions are benefitting students and in turn, society. It must first be determined whether the educational results are useful to society to achieve standards of success. The process of achieving educational outcomes cannot be viewed as a single entity. It involves a system of interrelated parts that work together to achieve the same goal (Dick et al., 2015). Regarding virtual internships, that goal should be to educate virtual interns on how to seamlessly integrate into a working society.

Researchers have advocated that participating in internships are practical ways for college students to develop a foundation for their future careers (Rothman \& Sisman, 2016). Internships take on an experiential form of learning that integrates classroom theory to the world of work. Experiential learning activities can take on many other 
approaches such as fieldwork, practicums, and undergraduate research. Many secondary and post-secondary institutions have utilized the concept of experiential learning to foster student success (NACE, 2017). The experiential nature of internships allows students to learn by doing actual work that relates to their field of interest. The rise of virtual internships have given access to a population of career-driven students who are geographically bound in locations that do not offer many internship or other work-related opportunities for them (Guthrie \& McCracken, 2010). It also allows students who have scheduling restrictions due to various obligations a flexible alternative to gaining work experience.

Experiential learning has helped to provide students with an opportunity to learn new things, outside of the classroom. Studies have shown that the role of supervision in internships cannot be understated (Hart et al., 2017; Schwartz-Bechet, 2014; Sweitzer \& King, 2014). An important challenge that internship programs face is making sure that the intern's experience is educational (Stirling et al., 2017).

\section{Discussion of Theory in Findings}

Leadership is mainly defined as a process of intentional influence over others to structure or facilitate activities and relationships in a group or organization (Yukl, 2013). While there are numerous theories of leadership present today, House's (1971) path-goal theory of leadership (PGTL) was utilized as the theoretical framework for this study. PGTL has become popular among researchers interested in the effects of this theory in the work environment (Bolen, 2015; Haney, 2017; Larson, 2015). PGTL is a dyadic theory that addresses the effects of superiors on subordinates. It promulgates the idea that leaders adjust their style of behavior to the specific environment they are working in and 
the employees that they are working with. A characteristic that is important to note in this study is that none of the eSupervisors had on-site employees or interns that they were supervising. Therefore, it is quite possible that their supervisory behaviors toward their eInterns were related to the specific context of a virtual environment as opposed to their preference in leadership styles.

There are three basic steps that PGTL follows:

1. Determine the employee and environmental characteristics

2. Select a leadership style

3. Focus on motivational factors that will help the employee succeed (Clark, 2013).

The first step in PGTL requires supervisors to figure out how employees will be able to work in a given context. In each case, the eSupervisors had different on-site work responsibilities and tasked their eInterns with assignments that could be completed virtually, that would supplement their on-site work objectives. The eSupervisors were all aware that their eInterns were students and had multiple responsibilities, so they made sure to adhere to the 10-hour per week working rule set by the VSFS management team.

The next step, requires a leadership style to be selected. There were a couple of eSupervisors who discussed an evolution in their management tactics with their eInterns based on prior experiences. For example, eSupervisor Two was more directive in his leadership approach during his first year supervising eInterns, but then decided to take on a "hands-off" approach the next year to empower them to be self-starters. Furthermore, eSupervisor Three had to evolve his leadership tactics because he took on eInterns who were not initially interested in the position. He gave them more authority and creative 
ownership over their work than in previous years to encourage a supportive and participative work environment. This exhibits a conscious effort in selecting leadership styles that were thought would be more effective in supervising their eInterns.

Finally, the last step requires leaders to find different ways of motivating their employees to foster success in the workplace. In discussing how each eSupervisor interacted with their eInterns, eSupervisor Three was only one who mentioned that he incorporated certain tactics that would make his eInterns feel welcome in a virtual environment. The others relied heavily on their eInterns self-motivated interests to get the job done. Whether initiated by the eSupervisor or not, there are various motivational factors that can come into play when helping an employee succeed. PGTL proposes that a follower's motivations are based on three assumptions: 1) if effort is given, the goal can be achieved; 2) if the goal is achieved, there will be a reward; and 3) the reward is valuable. For those eInterns who were self-motivated, their focus was on achieving a valuable reward at the end of the internship. In discussing with the past eInterns, many of them did find value in the rewards that were achieved through self-motivation.

\section{Assertions}

Case One. Only one eSupervisor exhibited directive path-goal clarifying leader behavior, with a focus on giving directions and providing structure. Directive path-goal clarifying lead behavior emphasizes giving direction and clarifying expectations (Yukl, 2006). eSupervisor One seemed to use a more directive approach to leadership as she mainly wanted her eInterns to learn the art of efficiency. eSupervisor One's directive approach to managing her eInterns brought about a couple of different factors in Case One: the value of time management, the value of proficiency, the value of self- 
motivated interests, and the need for creativity. From eSupervisor One's responses, it seemed as if proficiency, time management, and self-motivated interests were intentional outcomes from her leadership style and need for creativity was an indirect outcome that stemmed from her leadership. As stated, eSupervisor One wanted her eInterns to learn how to work efficiently. All of the eInterns in this case (both past and present) had some mention of the feedback that eSupervisor One delivered to ensure that their work met her standards. Albeit, this sort of leadership approach brought about an eIntern's want to try start trying things differently.

Case Two. House (1996) defines achievement oriented behavior as encouraging performance excellence in subordinates by raising their confidence levels and inspiring them to meet challenging goals. eSupervisor Two was classified as using an achievement-oriented style because of certain factors that emerged from each of the other participants in this case: the value of feeling appreciated, the value of opportunities, the value of empowerment, and the value of growth. eSupervisor Two was concerned with his eInterns learning how to be self-starters and independent workers. Their rewards for doing so ended up being a "promotion," a stacked resume, and in some cases, a great letter of reference. At the onset, it seemed as if eSupervisor Two took on a very nonchalant role in managing his eInterns; however, their overall feedback for him pointed in a different direction. This nonconventional way of achievement-oriented leadership contends that there are different ways that a leader can motivate his subordinates. In this case, it was a hands-off approach that was used to foster independent-action among those eInterns who had the drive to succeed, which was then followed by managerial support to enhance their success. 
Case Three. eSupervisor Three took on a more supportive and participative leadership approach with his eInterns, however, this could be attributed to the fact that he had to recruit them for his team this year. Supportive leader behavior has been seen to reduce stress and alleviate frustration for subordinates (House \& Mitchell, 1974, while participative leader behavior encourages subordinate participation in decision-making (House, 1996). eSupervisor Three wanted his eInterns to experiment and learn how to make mistakes, all the while having creative input and ownership over their projects. eSupervisor Three found himself in a situation where he started off as a supportive leader, but leaned toward being a participative leader later on. However, eSupervisor Three might not have fully evolved into a participative leader until his third cycle of eInterns, which is the cycle of students that were interviewed for this study. Evidence from this assertion stems from the graduates from his previous intern cycle who did not make mention of participating in any of the decision-making processes. Also, eSupervisor Three mentioned his evolution in leadership tactics as well. His previous eInterns valued accountability and flexibility, while his current ones valued creativity and community. eSupervisor Three might have moved towards a participative role due to the fact that he had to recruit his eInterns and wanted to find a way to motivate them to sign up for his program. In analyzing his previous leadership role, eSupervisor Three seemed to have taken on a mix between a participative and supportive leadership role.

Case Four. eSupervisor Four took on a leadership role that was kind of difficult to place as she was not necessarily directive, nor was she overwhelmingly supportive either. She did not consult with her eInterns on decision-making operations and she did not seem to offer up rewards for completing their tasks. It was finally concluded that 
eSupervisor Four did not fall within any specific leader behavior as she inadvertently used all four. According to PTGL, it is a leader's responsibility to select behaviors that will increase a follower's motivation to complete a task (House, 1971); this theory does not state that a leader must adhere to one specific style of leadership. eSupervisor Four did instruct her eInterns on what needed to be done but, as you saw with the findings from her eInterns in Chapter Four, there was still a lack of structure and guidance on her part. eSupervisor Four also wanted her eInterns to be independent workers who came up with their own ideas and grew in their skills. She "supported" and "encouraged" them by letting them rides their own bikes; no training wheels attached. Her eInterns were rewarded for completing their tasks by knowing that they were accomplishing something on their own, without any guidance from their superiors. The main factors that were valued by her eInterns were taking risks, flexibility, taking initiative, and autonomy.

\section{The Learning Cycle}

While it was not made apparent that any one of the eSupervisors or eInterns actively engaged in Kolb and Fry's (1975) entire learning cycle, one can debate that these experiences may require more time to flow through the entire cycle because of their virtual context. In this four-stage cycle, Kolb and Fry (1975) maintain that learning can begin at any stage, but must follow-through the entire sequence to be effective. Out of the four stages: concrete experience, reflective observation, abstract conceptualization, and active experimentation, it seemed as though engaging in reflective observation had not been followed-through by many of the eInterns. An indicator of this impression was hearing eInterns exclaim during their interviews that some of the items discussed were things they had not realized before or were difficult to answer because they were not 
thinking about it. For example, when asked to describe what did she want to learn more out of the virtual internship experience, Student 1A replied, "I'm not too sure..." (Individual interview, March $2^{\text {nd }}, 2018$ ). When answering the same question, Student 4A also stated that, "Nothing stands out as far as what more I can learn" (Individual interview, February $28^{\text {th }}, 2018$ ). When describing the issues that she had working in a virtual environment, Student 3A stated that she has not brought them up to her eSupervisor because, "I guess I didn't even realize it until now that it was something I was feeling" (Individual interview, March $2^{\text {nd }}, 2018$ ).

This notion of not engaging in reflective observation might also be premature as the eInterns were still in their program and could possibly engage in reflective observation at the end of their internship. The management team at VSFS sends out a survey to all participants at the end of the program, so this could possibly engage the eInterns in reflective observation as well. The past eInterns (graduates) were interviewed under a different protocol, so it is difficult to say whether they have engaged in reflective observation or not.

\section{Implications}

The findings from this study imply that there are a number of ways in which eSupervisors can contribute to the learning growth of eInterns. These contributions will vary depending on the eInterns, the environmental characteristics, the leadership approach taken, and the motivational factors involved. The significance in applying this research today falls in line with the booming growth of distance education programs the trend of incorporating technology in the classrooms. The results generated from this study will attract attention to the perceived educational outcomes that eSupervisors are 
working towards with their eInterns. The results that stem from this study also contribute to the literature in the areas concerning leadership methods that eSupervisors are utilizing with eInterns, House's (1971) path-goal theory of leadership, the lack of research on the effects of eSupervisory relationships with eInterns, and experiential learning opportunities for the non-traditional student population.

\section{Conclusions}

Qualitative research helps bring awareness to the meaning of what people have constructed about their experiences. The quintain in this study was the learning that results from participating in a virtual internship and how it is influenced by supervisory styles. Every experience is educational; whether it is negative or positive, it is something that has enlightened your senses to new possibilities. In addition, every experience is an educational contribution to learning outcomes. Whether a supervisor realizes it or not, they are teaching their employees something. Many of the interns described learning experiences that catered to their actual jobs, of course. But, what was of interest was what was learned without expectations - the independence, the creativity, the recognitions of certain needs, etc. Research has speculated on the potential that virtual internships have to support the development of cognitive processes and social aspects of professional development. Many eInterns learned how to think differently in terms of how to manage their time in a flexible work environment and how to hold themselves accountable for their actions. Other eInterns learned how important communication was to fostering a relationship with their eSupervisors. These experiences have all helped to shape how these eInterns interact or will interact with superiors and subordinates alike in their working environments. 


\section{Limitations}

A primary limitation to this study was the lack of research available on how supervisory relationships effect the learning that occurs in virtual internships. Although these limitations should be taken into consideration, it should not overlay the outcomes of this study. It is anticipated that the results of this study will inspire more research on virtual internships to come.

\section{Recommendations for Future Research}

The following recommendations for future research are based on the results from this study in an attempt to further explore the research quintain as it relates to learning outcomes as well as extend the theoretical framework:

1. Conduct a study with a focus on how eSupervisors compare in their leadership behaviors when supervising on-site employees or interns versus eInterns

2. Conduct a study with a focus on how eInterns engage in Kold and Fry's (1975) four-stage learning cycle

3. Conduct a study that analyzes how eInterns rate their eSupervisors in regards to meeting their learning needs and how eSupervisors rate their eInterns in regards to learning the tasks at hand.

\section{Recommendations for Practice}

The following recommendations for practice are based on findings from the results from this study in an attempt to foster a virtual internship experience that promotes student success:

1. Establish and nurture a community for virtual interns to allow for a more supportive work environment 
2. Establish a set of guidelines that not only inform virtual interns of performance expectations, but of internship goals, as well

3. Acknowledge the contributions that virtual interns bring to the work environment

4. Give virtual interns a chance to prove their worth

5. Communicate effectively and efficiently and allow for alternate methods of communication via telecommunication means. 


\section{REFERENCES}

Acai, A., Cowan, V., Doherty, S., Sharma, G., \& Thevathasan, N. (2014). Exploring the role of the university student as an experiential learner: Thoughts and reflections from the 2013 cohort of 3M National student fellows. Collected Essays on Learning \& Teaching, 7(2), 1-11.

Afshari, M., Siraj, S., Ghani, M. F. A., \& Afshari, M. (2011). Leadership and creativity. Australian Journal of Basic and Applied Sciences, 5(10), 1591-94.

Ahmed, Z. \& Nawaz, A. \& Khan, I. (2016). Leadership theories and styles: A literature review. Journal of Resources Development and Management, 16, 1-7.

Ahsan, S. M., \& Hassan, A. (2013). Increasing virtual education effectiveness by quantifying student learning trajectories and virtual internships. Life Science Journal, 10(10s).

Aldas, T., Crispo, V., Johnson, N., \& Price, T. A. (2010). Learning by doing: The Wagner Plan from classroom to career. Peer Review, 12(4), 24-28.

Allen, I. E., \& Seaman, J. (2010a). Class differences: Online education in the United States, 2010. Babson Survey Research Group, Retrieved August 11, 2015 from, http://files.eric .ed.gov/fulltext/ED529952.pdf

Allen, I. E., \& Seaman, J. (2010b). Learning on demand: Online education in the United States, 2009. Babson Survey Research Group. Retrieved August 11, 2015 from, http://www.onlinelearningsurvey.com/reports/learning-on-demand.pdf

Allen, I. E., \& Seaman, J. (2015). Grade level: Tracking online education in the United States, 2014. Babson Survey Research Group and Quahog Research Group, LLC. Retrieved October 18, 2015 from, http://onlinelearningconsortium.org/read/survey-reports-2014/

Ambrose, S. A., \& Poklop, L. (2015). Do students really learn from experience?. Change, 47(1), 54-61. doi:10.1080/00091383.2015.996098

Ambrose, S. A., Bridges, M. W., DiPietro, M., Lovett, M. C., \& Norman, M. K. (2010). How learning works: Seven research-based principles for smart teaching (1st ed.). San Francisco, CA: Jossey-Bass.

Anderson, J. R. (1995). The architecture of cognition. Cambridge, MA: Harvard University Press.

Antonakis, J., Cianciolo, A., \& Sternberg, R. (2004). The nature of leadership. Thousand Oaks: Sage Publications. 
Archer, M., Decoteau, C., Gorski, P., Little, D., Porpora, D., Rutzou, T., Smith, C., Steinmetz, G., \& Vandenberghe, F. (2016). What is critical realism? Retrieved September 2, 2017 from http://www.asatheory.org/current-newsletteronline/what-is-critical-realism

ASU Now: Access, Excellence, Impact. (2016, February 5). Virtual internship takes innovation to the next level. Retrieved from https://asunow.asu.edu/20160205virtual- internship-takes-innovation-next-level

Austin, M. J., \& Rust, D. Z. (2015). Developing an experiential learning program: Milestones and challenges. International Journal of Teaching \& Learning In Higher Education, 27(1), 143-153.

Baldwin, S. C., Buchanan, A. M., \& Rudisill, M. E. (2007). What teacher candidates learned about diversity, social justice, and themselves from service-learning experiences. Journal of Teacher Education, 58(4), 315-327. doi:10.1177/0022487107305259

Barratt, M., Choi, T. Y., \& Li, M. (2011). Qualitative case studies in operations management: Trends, research outcomes, and future research implications. Journal of Operations Management, 29(4), 329-342.

Bass, B. M. (1990). Bass \& Stogdill's handbook of leadership, 3rd edition. New York, NY: The Free Press.

Bass, B .M. (1995). Theory of transformational leadership redux. The Leadership Quarterly 6(4), pp. 463-478. doi: https://doi.org/10.1016/1048-9843(95)90021-7

Baškarada, S. (2014). Qualitative case studies guidelines. The Qualitative Report, 19(40), $1-25$.

Bayerlein, L. (2015). Curriculum innovation in undergraduate accounting degree programmes through "virtual internships". Education + Training, 57(6), 673-684. doi:10.1108/ET-09-2014-0110

Beatty, P. \& Willis, G. 2007. Research synthesis: The practice of cognitive interviewing. PublicOpinion Quarterly, Vol. 71, No. 2, pp. 287-311.

Best Counseling Degrees. (2017, May). What is the difference between a practicum and an internship? Retrieved from http://www.bestcounselingdegrees.net/faq/what-isthe- difference-between-a-practicum-and-an-internship/.

Bhaskar, R. (1975). A Realist Theory of Science. London: Verso. 
Boehe, D. M. (2016). Supervisory styles: a contingency framework. Studies in Higher Education, 41(3), 399-414. doi:10.1080/03075079.2014.927853

Bogdan, R.C., \& Biklen, S.K. (2007). Qualitative research for education: An introduction to theories and methods. Boston, MA: Pearson.

Bolen, H. M. (2015). Applying leadership theory to the work-family interface:

Examining the interactive effects of family supportive supervisor behaviors and leader-member exchange quality (Order No. AAI3581836). Available from PsycINFO. (1707078070; 2015-99160-518). Retrieved from http://search.proquest.com.ezproxylocal.lib rary.nova.edu/d ocview/1707078070?accountid=6579

Boling, E. C., Hough, M., Krinsky, H., Saleem, H., \& Stevens, M. (2012). Cutting the distance in distance education: Perspectives on what promotes positive, online learning experiences. The Internet and Higher Education, 15(2), 118-126.

Bourque, L. B., \& Fielder, E. P. (2003). How to conduct self-administered and mail surveys $\left(2^{\text {nd }}\right.$ ed. $)$. Thousand Oaks, CA: SAGE Publications.

Boyington, B. (2015, March 31). Understand the differences between a co-op, internship. Retrieved from https://www.usnews.com/education/best-colleges/articles/201 5/03/31/understand-the-differences-between-a-co-op-internship.

Brownell, J., \& Swaner, L. (2010). Five high-impact practices: Research on learning outcomes, completion, and quality. Washington, DC: Association of American Colleges \& Universities.

Burak, O. C. \& Bashshur, M. R.. (2013). Followership, leadership and social influence. Leadership Quarterly, 24(6), 919-934.

Burns, J. M. (1978). Leadership. New York: Harper \& Row.

Canadian Council on Learning (CCL). (2008). The benefits of experiential learning. Ottawa: CCL.

Cantor, J. (1995). Experiential learning in higher education: Linking classroom and community.ASHE-ERIC Higher Education Report No. 7.

Carlyle, T. (1840). On Heroes, Hero-Worship, and the Heroic in History. The Project Gutenburg Ebook.

Carson, P., Carson, K., \& Roe, C. (1993). Social power bases: A metaanalytic examination of interrelationships and outcomes. Journal of Applied Social Psychology, 23(14), 1150-1169. 
Casner-Lotto, J., \& Barrington, L. (2006). Are they really ready to work? The Conference Board, Corporate Voices for Working Families, Partnership for 21st Century Skills, and Society for Human Resource Management. Retrieved from http://www.p21.org/storage documents/FINAL_REPORT_PDF09-29-06.pdf.

Cater, M., \& Jones, K. Y. (2014). Measuring perceptions of engagement in teamwork in youth development programs. Journal of Experiential Education, 37, 176-186.

Central European Review (CER) (2017) CER's Virtual Internship Programme. Available at http://www.ce-review.org/_internships.html (accessed June 2017).

Cherry, K. (2012). Leadership Theories - 8 Major Leadership Theories. Retrieved September, 2017 fromhttp://psychology.about.com/od/leadership/p/ leadtheories.htm.

Chesler, N. C., Arastoopour, G., D'Angelo, C. M., Bagley, E. A., \& Shaffer, D. W. (2013). Boost Their Game. ASEE Prism, 23(2), 53.

Cicco, G. (2014). Building effective supervisory relationships in the online counseling course: Faculty and student responsibilities. Journal on School Educational Technology, 10(2), 1-8. Retrieved from http://search.proquest.com.ezproxylocal.library.nova. edu/docview/1826534676?accountid=6579.

Clark, D. (2013, October 31). Path-Goal theory of leadership. Retrieved from http://www.nwlink.com/ donclark/leader/lead_path_goal.html.

Clemmons, O. (2015). Interning From Home. TD: Talent Development, 69(6), 14.

Coker, P. (2010). Effects of an experiential learning program on the clinical reasoning and critical thinking skills of occupational therapy students. Journal of Allied Health, 39(4). 280-286.

Coker, J. J., Heiser, E., Taylor, L., \& Book, C. (2017). Impacts of experiential learning depth and breadth on student outcomes. Journal of Experiential Education, 40(1), 5-23. doi:10.1177/1053825916678265

Cornelius, S., Medyckyj-Scott, D., Forrest, D., Williams, A., \& Mackaness, W. (2008). The virtual placement: An alternative to the traditional work placement in the geographical sciences? Journal of Geography in Higher Education, 32(2), 287302. Retrieved from http://search.proquest.com.ezproxylocal.library .nova.edu/docview /61959353 accountid $=6579$. 
Crawford, S. D., Couper, M. P., \& Lamias, M. J. (2001). Web surveys: Perceptions of burden. Social Science Computer Review, 19, 146-162.

Crawley, A. (2012). Supporting Online Students. A Guide to Planning, Implementing and Evaluating Services. San Francisco, CA: Jossey-Bass.

Creswell, J. W. (2015). Educational research: Planning, conducting, and evaluating quantitative and qualitative research (5th ed.). Upper Saddle River, NJ: Pearson.

Cruz, M. A., Henningsen, D. D. \& Smith, B. A. (1999). The impact of directive leadership on group information sampling, decisions, perceptions of the leader. Communication Research, 26, 349-70.

Cunningham, G.B., Sagas, M., Dixon, M., Kent, A. \& Turner, B.A. (2005). Anticipated career satisfaction, affective occupational commitment, and intentions to enter the sport management profession. Journal of Sport Management, 19(1), 43-47.

D’Amato, L. G., \& Krasny, M. E. (2011). Outdoor adventure education: Applying transformative learning theory to understanding instrumental learning and personal growth in environmental education. The Journal of Environmental Education, 42(4), 237-254. Doi:10.1080/00958964.2011.581313

Dane, F. C. (2010). Evaluating research: Methodology for people who need to read research. Thousand Oaks, CA: Sage.

Dansereau, F., Yammarino, F. J., Markham, S. E., Alutto, J. A., Newman, J., Dumas, M., \& Nachman, S. A. (1995). Individualized Leadership: A New Multiple-level Approach. The Leadership Quarterly, 6(3), 413-450.

Darius, P.L., Portier, K.M. \& Schrevens, E. (2007). Virtual experiments and their use in teaching experimental design. Int. Statist. Rev., 75, 281-294.

Dewey, J. (1916). Democracy and Education. NY: Free Press

Dewey, J. (1938). Education and Education. NY: Free Press

Dewey, R. E. (1971). Accredited experiential education: Some definitions. The Urban and Social Change Review, 5(1), 10-15.

Dick, W., Carey, L., \& Carey, J. O. (2015). The systematic design of instruction (8th ed.). Upper Saddle River, NJ: Pearson

Divine, R.L., Linrud, J.K., Miller, R.H \& Wilson, J.H. (2007). Required internship programs in marketing: Benefits, challenges and determinants of fit. Marketing Education Review, 17 (2), 45-52. 
Donovan, T., Porter, R., \& Stellar, J. (2010). Experiencing success: Some strategies for planning the program. New Directions for Teaching and Learning, 2010(124), 8994. doi: $10.1002 / \mathrm{tl} .426$

Doyle, A. (2017, June 22). What is telecommuting? - Definition, pros and cons. Retrieved from https://www.thebalance.com/what-is-telecommuting-2062113

Drewery, D., Nevison, C. \& Pretti, T. J. (2016). The influence of cooperative education and reflection upon previous work experiences on university graduates' vocational self-concept. Education+Training, 58(2), pp. 179-192.

Easton, G. (2010). Critical realism in case study research. Industrial Marketing Management, 39(1), 118-128.

Edmonds, W. A., \& Kennedy, T. D. (2012). An applied reference guide to research designs: Quantitative, qualitative, and mixed methods. Thousand Oaks, CA: Sage.

Ekvall, G. \& Arvonen, J. (1991). Change-centred leadership: An extension of the twodimensional model. Scandinavian Journal of Management, 7(1), pp. 17-26.

Ellis, M. V., Berger, L., Hanus, A. E., Ayala, E. E., Swords, B. A., \& Siembor, M. (2014). Inadequate and Harmful Supervision Descriptor Ratings [Database record]. Retrieved from PsycTESTS. doi: http://dx.doi.org/10.1037/t34123-000

English, W. D., \& Lewison, D. M. (1979). Marketing internship programs: Striking out in the academic ballgame. Journal of Marketing Education, 1(2), 48-52.

Eppler, M. A., Ironsmith, M., Dingle, S. H., \& Errickson, M. A. (2011). Benefits of service learning for freshman college students and elementary school children. Journal of Scholarship of Teaching and Learning, 11(4), 102-115.

Evans, M. G. (1970). The effects of supervisory behavior on the path-goal relationship. Organizational Behavior and Human Performance, 5, 277-298.

Eyler, J. T. (1992). Comparing the impact of two internship experiences on student learning. Journal of Cooperative Education, 29, 41-52.

Fan, W. \& Yan, Z. (2010). Factors affecting response rates of the web survey: A systematic review. Computers in Human Behavior, 26, 132-139. doi:10.1016/j.chb.2009.10.015

Feinberg, W., \& Soltis, J. F. (2009). School and society. 5th Ed. New York: Teachers College Press. 
Fernald, P., \& Goldstein, G. (2013). Advanced internship: A high-impact, low-cost, super-capstone course. College Teaching, 61, 3-10.

Fiedler, F.E. (1964). A contingency model of leadership effectiveness. In L. Berkowitz (ed), Advances in Experimental Social Psychology, New York: Academic Press.

Fiedler, F. E. (1971). Validation and extension of the contingency model of leadership effectiveness: A review of empirical findings. Psychological Bulletin, 76(2), 128148. http://dx.doi.org/10.1037/h0031454

Fiedler, F. E., \& Garcia, J. E. (1987). New approaches to effective leadership: Cognitive resources and organizational performance. Oxford, England: John Wiley \& Sons.

Finley, A., \& McNair, T. (2013). Assessing underserved students' engagement in highimpact practices. Washington, DC: Association of American Colleges and Universities.

Flexjobs. (2017, September). The 2017 state of telecommuting in the U.S. employee workforce. Retrieved from https://www.flexjobs.com/2017-State-ofTelecommuting-US/

Florida International University. (2016). FIU BeyondPossible2020. Retrieved from http://stratplan.fiu.edu/docs/Strategic\%20Plan.pdf

Franks, P. C., \& Oliver, G. C. (2012). Experiential learning and international collaboration opportunities: Virtual internships. Library review, 61(4), 272-285.

Fyfe, T., \& Dennett, L. (2014). Building capacity in systematic review searching: A pilot program using virtual mentoring. Journal of the Canadian Health Libraries Association, 33(1), 12-16.

Gault, J., Leach, E. \& Duey, M. (2010). Effects of business internships on job marketability: The employers' perspective. Education+Training, 52(1), 76-88.

Gault, J., Redington, J., \& Schlager, T. (2000). Undergraduate business internships and career success: Are they related? Journal of Marketing Education, 22, 45-53.

Gavigan, L. (2010). Connecting the classroom with real-world experiences through summer internships. Peer Review, 12(4), 15-19.

General Accounting Office (GAO). (1990). Case study evaluations. Washington, DC.

Global Workplace Analytics. (2013). Latest telecommuting statistics. Retrieved from http://globalworkplaceanalytics.com/telecommuting-statistics. 
Graen, G. B., \& Uhl-Bien, M. (1995). Relationship-based approach to leadership: Development of leader-member exchange (LMX) theory of leadership over 25 years: Applying a multi-level multi-domain perspective. The Leadership Quarterly 6(2), pp. 219-247.

Grasgreen, A. (2012). Résumé-builder or rip-off? Insider Higher Ed. Retrieved from www.insidehighered.com/news/2012/02/03/growth-short-term-internships-overacademicbreas

Greer, T.W. (2013). Maximize the internship experience for employers and students. T\&D Magazine, American Society for Training \& Development, 70-72.

Grovers, R. M., Fowler, F. J. Jr., Couper, M. P., Lepkowski, J. M., Singer, E., Tourangeau, R. (2009). (2nd Ed.). Survey Methodology. Hoboken, New Jersey: John Wiley \& Sons, Inc.

Groves, D. L., Howland, B., Headly, F., \& Jamison, D. (1977). Relevance in the classroom and curriculum. College Student Journal, 11, 259-261.

Gu, J., He, C., \& Liu, H. (2017). Supervisory styles and graduate student creativity: the mediating roles of creative self-efficacy and intrinsic motivation. Studies in Higher Education, 42(4), 721-742. doi:10.1080/03075079.2015.1072149

Guile, D., \& Griffiths, T. (2001). Learning through work experience. Journal of Education and Work, 14(1), 113-131.

Guthrie, K. L., \& Jones, T. B. (2012). Teaching and learning: Using experiential learning and reflection for leadership education. New Directions for Student Services, 2012, 53-63. doi:10.1002/ss.20031

Guthrie, K. L., \& McCracken, H. (2010). Reflective pedagogy: Making meaning in experiential based online courses. Journal of Educators Online, 7(2), 21. Retrieved from_http://search.proquest.com.ezproxylocal.library.nova. edu/docview/_815957201 ?accountid=6579.

Halpin, A. W. \& Winer, B. J. (1957). A factorial study of the leader behavior descriptions. In Stogdill, R.M. \& Coons, A.E. (Eds.). Leader behavior: Its description and measurement. Columbus, $\mathrm{OH}$ : Bureau of Business Research, Ohio State University.

Haney, D. D. (2017). Perceptions of leadership and employee performance in child welfare agencies (Order No. 10607168). Available from ProQuest Dissertations \& Theses Global. (1943998858). Retrieved from 
http://search.proquest.com.ezproxylocal.library.nova.edu/ docview/1943998858? accountid=6579.

Harber, C. (2014). Education and international development: Theory, practice and issues. United Kingdom: Symposium Books.

Hart, P. D. Research Associates, Inc. (2008). How should colleges assess and improve student learning? Employers' views on the accountability challenge. Association of American Colleges and Universities. Retrieved from http://www.eric.ed.gov/ contentde livery/servlet/ERICServlet?accno=ED499718.

Hart, M., Kremin, J., \& Pasewark, W. R. (2017). Growing up: How audit internships affect students' commitment and long-term intentions to work in public accounting. Issues in Accounting Education, 32(2), 47-63. doi:10.2308/iace51431

Hart Research Associates. (2016). Recent trends in general education design, learning outcomes and teaching approaches. Washington, DC: Association of American Colleges \& Universities.

Heafner, T. L., Petty, T. M., \& Hartshorne, R. (2012). University supervisor perspectives of the remote observation of graduate interns. Journal of Computing in Higher Education, 24(3), 143-163. doi:http://dx.doi.org.ezproxylocal.library.nova.edu/10 .1007/s12528-012-9063-1

Heerwegh, D., \& Loosveldt, G. (2003). An evaluation of the semiautomatic login procedure to control web survey access. Social Science Computer Review, 21, 223-234.

Heberlein, T. A., \& Baumgartner, R. (1978). Factors affecting response rates to mailed questionnaires: A quantitative analysis of the published literature. American Sociological Review, 43, 447-462.

Heinrich, W. F., Habron, G. B., Johnson, H. L., \& Goralnik, L. (2015). Critical thinking assessment across four sustainability-related experiential learning settings. Journal of Experiential Education, 38, 373-393.

Henman, L. D. (2011). Leadership: Theories and Controversies. Retrieved from http://henmanperformancegroup.com.

Hergert, M. (2009). Student perceptions of the value of internships in business education. American Journal of Business Education, 2(8), 9-14.

Hersey, P., Blanchard, K., \& Natemeyer, W. (1979). Situational leadership, perception, and the impact of power. Sage Journals, 4(4), pp. 418-428. 
HeySuccess. (2017, August). Virtual internship program - United Nations University, Campus Computing Centre (UNU). Retrieved from https://www.heysuccess.com /opportunity/Virtual-Internship-Program-United-Nations-University-CampusComputing-Centre-(UNU-C3)-20274.

Hiltebeitel, K. M., Leauby, B. A. \& Larkin, J.M. (2000). Job satisfaction among entrylevel accountants. CPA Journal, 70(5), 76-79.

Hirsch, P., \& Lloyd, K. (2005). Real and Virtual Experiential Learning on the Mekong: Field Schools, e-Sims and Cultural Challenge. Journal of Geography In Higher Education, 29(3), 321-337. doi:10.1080/03098260500290892

House, R. J. (1971). A path-goal theory of leader effectiveness. Administrative Science Quarterly, 16, 321-338.

House, R. J. (1996). Path-Goal theory of leadership: Lessons, legacy, and a reformulated theory. Leadership Quarterly, 7, 323-52.

House, R., \& Aditya, R. (1997). The social scientific study of leadership: Quo Vadis? Journal of Management, 23, 409-474.

House, R. J. \& Mitchell, T. R. (1974) Path-goal theory of leadership. Journal of Contemporary Business, 3, 81-97.

House, R. J., \& Shamir, B. (1993). Toward the integration of transformational, charismatic, and visionary theories. In M. M. Chemers, \& R. Ayman (Eds.), Leadership theory and research: Perspectives and direction, (pp. 81-107). San Diego, CA: Academic Press.

Howell, S. L., Williams, P. B. \& Lindsay, N. K. (2003, Fall). Thirty-two trends affecting distance education: An informed foundation for strategic planning. Online Journal of Distance Learning Administration, 6(3). Retrieved from http://www.westga.edu.proxy. lib.fsu.edu/ distance/ojdla/fall63/howell63.html.

Hursch, B., \& Borzak, L. (1979). Toward cognitive development through field studies. Journal of Higher Education, 50, 63-77.

Intern Queen Inc. (2017). Virtual internships - virtual placement programs. Retrieved August 27, 2017, from https://www.internqueen.com/internships/virtual

Internships.com. (2017). Find 2017 remote internships. Retrieved August 27, 2017, from http://www.internships.com/virtual. 
Ives, B., \& Obenchain, K. (2006). Experiential education in the classroom and academic outcomes: For those who want it all. Journal of Experiential Education, 29, 6177.

Jackson, D., Sibson, R. and Riebe, L. (2014). Undergraduate perceptions of the development of team-working skills. Education + Training, 56(1), 7-20.

James, William (1890). The Principles of Psychology. New York: Henry Holt.

Jarvis, P. (1987). Adult learning in the social context. London: Routledge.

Jeske, D., \& Axtell, C. (2014). e-Internships: Prevalence, characteristics and role of student perspectives. Internet Research, 24(4), 457-473.

Jiusto, S., \& Dibiasio, D. (2006). Experiential learning environments: Do they prepare our students to be self-directed life-long learners? Journal of Engineering Education, 95(3), 95-204.

John, O. \& Robins, R. (1993). Gordon Allport: Father and critic of the five-factor model. In Craik, K., Hogan, R., \& Wolfe, R. Fifty Years of Personality Psychology (pp. 215-236). New York: Plenum Press.

Jones, E. (2002). Transforming the curriculum: Preparing students for a changing world. ASHE-ERIC Higher Education Report, 29(3).

Kaufman, R., Watkins, R., \& Leigh, D. (2001). Useful educational results. Lancaster, PA: Proactive Publishing.

Katula, R. A., \& Threnhauser, E. (1999). Experiential education in the undergraduate curriculum. Communication Education, 48(3), 238. doi: 10.1080/03634529 909379172

Kellerman, B. (2007). What every leader needs to know about followers. Harvard Business Review. Retrieved from https://hbr.org/2007/12/what-every-leaderneeds-to-know-about-follower.

Kilgo, C., Sheets J., \& Pascarella E. (2015). The link between high-impact practices and student learning: Some longitudinal evidence. Higher Education, 69, 509-525.

Kim, C., Yang, Z., \& Lee, H. (2015). Parental Style Measure [Database record]. Retrieved from PsycTESTS. doi: http://dx.doi.org/10.1037/t43606-000

Knemeyer, A. M., \& Murphy, P. R. (2002). Logistics internships: Employer and student perspectives. International Journal of Physical Distribution \& Logistics Management, 32, 135-152. 
Knouse, S., Tanner, J., \& Harris, E. (1999). The relation of college internships, college performance, and subsequent job opportunity. Journal of Employment Counseling, 36, 35-43.

Kohs, S. C., \& Irle, K. W. (1920). Prophesying army promotion. Journal of Applied Psychology, 4, 73-87.

Kolb, A. Y., \& Kolb, D. A. (2005). Learning styles and learning spaces: Experiential learning in higher education. Academy Management Learning \& Education, 4(2), 193-212.

Kolb, D. A. (1984). Experiential learning. Upper Saddle River, NJ: Prentice-Hall Inc.

Kolb. D. A., \& Fry, R. (1975). Toward an applied theory of experiential learning. In C. Cooper (ed.) Theories of Group Process, London: John Wiley.

Krohn, F. D. (1986). Time to blow the whistle on internships. Marketing Educator, Marketing Educator, 1(2), 1-2.

Kuh, G. (2008). High-impact educational practices: What they are, who has access to them, and why they matter. Washington, DC: Association of American Colleges and Universities.

Kuh, G. D., \& O’Donnell, K. (2013). Ensuring quality and taking high-impact practices to scale. Washington, DC: Association of American Colleges \& Universities.

Kuhn, T. S. (1962). The Structure of Scientific Revolutions. In Foundations of the Unity of Science 2(2). Chicago, Il: The University of Chicago Press.

Landis, E., Hill, D., Harvey, M. (2014). A synthesis of leadership theories and styles. Journal of Management Policy and Practice, 15(2), 97-100.

Larson, S. C. (2015). Path-goal leadership, organizational commitment, and turnover intention among generational cohorts (Order No. AAI3645520). Available from Sociological Abstracts. (1718065399; 201531074). Retrieved from http://search.proquest.com.ezproxy local.library.nova.edu/docvi ew/1718065399? accountid=6579

Lawler, E. E., \& Porter, L. W. (1967). The effect of performance on job satisfaction. Industrial Relations, 7, 20-28.

Leadership-Central. (2016). Leadership theories. Retrieved August 2017, from http://www.leadership-central.com/leadership-theories.html\#axzz4sK 6wE9Jn. 
Learn to be a leader. (2009). Leadership theories: An overview in everyday language. Retrieved August 2017, from http://www.learn-to-be-a-leader.com/leadership -theories.html.

Lewin, K. (1951). Field theory in social science. New York: Harper \& Row.

Lewin, K., Lippit, R. \& White, R.K. (1939). Patterns of aggressive behavior in experimentally created social climates. Journal of Social Psychology, 10, 271301.

Lian, L. K., \& Tui, L. G. (2012). Journal of Applied Business and Economics, 13(2), 5996.

Lincoln, Y. S., \& Guba, E. G. (1985). Naturalistic inquiry (Vol. 75). Sage.

LinkedIn. (2017). Virtual internship. Retrieved August 27, 2017, from https://www.linkedin.com/jobs/virtual-internship-jobs.

Littlepage, L., Gazley, B., \& Bennett, T. (2012). Service-learning from the supply side: Community capacity to engage students. Nonprofit Management and Leadership, 22(3), 305-320.

Liu, Y., Xu, J. and Weitz, B.A. (2011). The role of emotional expression and mentoring in internship learning. Academy of Management Learning and Education, 10(1), 94-110.

Lee, S., \& Dickson, D. (2010). Increasing student learning in the classroom through experiential learning programs outside the classroom. Journal of Hospitality and Tourism Education, 22(3), 27- 34. doi: 10.1080/10963758.2010.10696982

Lord, D.R., Sumrall, D. \& Sambandam, R. (2011). Satisfaction determinants in business internships. Interdisciplinary Journal of Contemporary Research in Business, 2(10), 1-22.

Lunenburg, F. (2011). Expectancy theory of motivation: Motivating by altering expectations. Iinternational Journal of Management, Business, and Administration, 15(1), 1-6.

Lunenburg, F. (2012). Power and leadership: An influence process. Iinternational Journal of Management, Business, and Administration, 15(1), 1-9.

McGregor, D. M. (2003). The human side of enterprise. New York: McGraw-Hill. 
Maertz, C. P., Jr., Stoeberl, P. A., \& Marks, J. (2014). Building successful internships: Lessons from the research for interns, schools, and employers. Career Development International,19, 123-142.

Mannheim, B., \& H. Halamish. (2008). Transformational leadership as related to team outcomes and contextual moderation. Leadership \& Organization Development Journal, 29(7), 617-30.

Mathooko, J. M. (2013). Leadership and organizational ethics: the three dimensional African perspectives. BMC Medical Ethics, 14(1 1), S2. http://doi.org/10.1186/1472-6939-14-S1-S2

Mayer, D., Aquino, K., Greenbaum, R., \& Kuenzi, M. (2012). Who displays ethical leadership, and why does it matter? An examination of antecedents and consequences of ethical leadership. Academy of Management Journal, (55)1, 151-171.

McCallum, J. (2013). Followership: The other side of leadership. Ivey Business Journal. Retrieved from https://iveybusinessjournal.com/publication/followership-theother-side-of-leadership/

McCarthy, P.R. \& McCarthy, H.M. (2006). When case studies are not enough: integrating experiential learning into business curricula. Journal of Education for Business, 81(2), 201-204.

McKeachie, W. J. (2002). McKeachie's teaching tips: Strategies, research, theory for college, and university teachers (11th ed.). Boston, MA: Houghton Mifflin.

McKnight, Z. (2013). Young, unpaid workers face thin protections under Ontario law. Toronto Star. Retrieved May 8, 2017 from http://www.thestar.com/news/ gta/2013/10/17/young_unpaid_workers_face_thin_protections_under_ ontario_law.html.

Meizrow, J. (1990). How critical reflection triggers transformative learning. In J. Meizrow (Ed.), Fostering critical reflection in adulthood (pp. 1-20). San Francisco, CA: Jossey-Bass Publishers.

Merriam-Webster. (2017). Supervisor. Retrieved from https://www.merriamwebster.com/dictionary/supervisor.

Meyer, L. L. (2016). Students at Oakland Unified Participate in Virtual Medical Internships. $T$ H E Journal, 43(1), 22-23. 
Miller, R., Rycek, R., \& Fritson, K. (2011). The effects of high-impact learning experiences on student engagement. Procedia - Social and Behavioral Sciences, 15, 53-59.

Milman, N. B., Posey, L., Pintz, C., Wright, K., \& Zhou, P. (2015). Online master's students' perceptions of institutional supports and resources: Initial survey results. Online Learning, 19(4), 45-66.

Moghaddam, J. M. (2011). Perceived effectiveness of business internships: Student expectations, experiences and personality traits. International Journal of Management, 28(4), 287-303.

Moore, D. T. (2010). Forms and issues in experiential education. In D. M. Qualters (ed.), Experiential education: Making the most of learning outside the classroom (pp. 3-14). San Francisco, CA: Jossey-Bass.

Mount Holyoke College. (2017, May). Student Projects: Research Practicum vs Research Project. Retrieved from https://www.mtholyoke.edu/irb/practicum.

Mulki, J., Bardhi, F., Lassk, F., \& Nanavaty-Dahl, J. (2012). Set up remote workers to thrive. Occupational outlook handbook (2011). Washington, D.C.: Bureau of Labor Statistics. Available at http://www.bls.gov/ooh/.

Narayanan, V.K., Olk, P.M.O. \& Fukami, C.V. (2010). Determinants of internship effectiveness: An exploratory model. Academy of Management Learning \& Education, 9(1), 61-80.

National Association of Colleges \& Employers (NACE). (2005). 2005 NACE Experiential Education Survey Executive Summary. Bethlehem, PA: Author. Retrieved from http://www.naceweb.org/info public/surveys.htm\#experiential.

National Association of Colleges and Employers (NACE). (2017, May). Job outlook 2016: The attributes employers want to see on new college graduates' resumes. Retrieved from http://www.naceweb.org/career-development/trends-andpredictions/job-outlook-2016-attributes-employers-want-to-see-on-new-collegegraduates-resumes/.

National Center for Education Statistics (2002, June). The condition of education 2002 (NCES 2002-025). Washington, DC: Author.

National Society for Experiential Education (NSEE). (1988). Eight principles of good practice for all experiential learning activities. Retrieved May 2017, from http://www.nsee.org/8-principles. 
National Survey of Student Engagement (NSSE). (2017). High-Impact Practices. Retrieved from http://nsse.indiana.edu/html/high_impact_practices.cfm.

Naylor, J. (1999). Management. Harlow, England: Prentice Hall.

Neukrug, E. (2012). The world of the counselor: An introduction to the counseling profession (4th ed.). Belmont, CA: Brooks/Cole.

Nolan, D. \& Temple Lang, D. (2007). Dynamic, interactive documents for teaching statistical practice. Int. Statist. Rev., 75, 295-321.

Northouse, P. (2013). Leadership Theory and practice (6 ${ }^{\text {th }}$ Ed). Thousand Oaks: Sage Publications, Inc.

O’Neil, N. (2010). Internships as a high-impact practice: Some reflections on quality. Peer Review, 12(4), 4-8.

Parcells, N. (2013, May). How to start your virtual internship program. Retrieved from https://techli.com/2013/05/how-to-start-your-virtual-internship-program/.

Parlett, M., \& Hamilton, D. (1976). Evaluation as illumination: A new approach to the study of innovative programmes. In G. V. Glass (Ed.), Evalua, tion studies review annual (Vol. 1, pp. 141-157). Beverly Hills, CA: Sage.

Parker III, E. E., Kilgo, C. A., Sheets, J. E., \& Pascarella, E. T. (2016). The differential effects of internship participation on end-of-fourth-year GPA by demographic and institutional characteristics. Journal of College Student Development, 57(1), 104109.

Patton, M. Q. (1990). Qualitative evaluation and research methods (2nd ed.). Newbury Park, CA: Sage.

Piaget, J. (1970). Science of education and the psychology of the child. New York: Viking.

Porter, L. W., \& Lawler, E. E. (1968). Managerial attitudes and performance. Homewood, IL: Dorsey Press.

Purdie, F., Ward, L., Mcadie, T., King, N., \& Drysdale, M. (2013). Are work-integrated learning (WIL) students better equipped psychologically for work post-graduation than their non-work-integrated learning peers? Some initial findings from a UK university. Asia-Pacific Journal of Cooperative Education, 14(2), 117-125.

Ramson, A. (2014). Service-learning: A tool to develop employment competencies for college students. Journal on Excellence in College Teaching, 25(1), 159-187. 
Retallick, M. S., \& Steiner, C. (2009). A model for implementing a college-wide experiential learning program in higher education. NACTA Journal, 53(1), 2-5.

Rosenberg, S., Heimler, R. \& Morote, E.-S. (2012). Basic employability skills: A triangular design approach. Education + Training, 54(1), 7-20.

Rothman, M., \& Sisman, R. (2016). Internship impact on career consideration among business students. Education + Training, 58(9), 1003-1013. doi:10.1108/ET-042015-0027.

Ruggiero, D., \& Boehm, J. (2016). Design and development of a learning design virtual internship program. International Review of Research in Open and Distributed Learning, 17(4), 105-120. Retrieved from http://search.proquest.com. ezproxylocal.library .nova.edu/docview/1826550983 ?accountid=6579.

Ruggiero, D., \& Boehm, J. (2017). Project-based learning in a virtual internship programme: A study of the interrelated roles between intern, mentor and client. Computers \& Education, 110116-126. doi:10.1016/j.compedu.2017.03.011

Ruth, S., \& Chaudhry, I. (2008). Telework: A productivity paradox? Internet Computing, IEEE, 12(6), 87-90.

San Jose State University (SJSU). (2017, August). Virtual internships. Retrieved from http://ischool.sjsu.edu/current-students/courses/internships/virtual-internships.

Sawyer, K. (2017). Keeping it real: The impact of HRD internships on the development of HRD professionals. Advances In Developing Human Resources, 19(2), 176189. doi:10.1177/1523422317695228

Schwartz-Bechet, B. (2014). Virtual supervision of teacher candidates: A case study. International Journal of Learning: Annual Review, 211-12.

Scogin, S. S., Kruger, C. J., Jekkals, R. E., \& Steinfeldt, C. (2017). Learning by experience in a standardized testing culture: Investigation of a middle school experiential learning program. Journal of Experiential Education, 40(1), 39-57. doi:10.1177/1053825916685737

Shadish, W. R., Cook, T. D., \& Campbell, D. T. (2002). Experimental and quasiexperimental designs for generalized causal inference. Boston, MA: Houghton Mifflin.

Shamir, B. (2007). From passive recipients to active co-producers: The roles of followers in the leadership process. In B. Shamir, R. Pillai, M. Bligh, \& M. Uhl-Bien 
(Eds.), Follower-centered perspectives on leadership: A tribute to J. R. Meindl. Stamford, CT: Information Age Publishing.

Sheehan, K. B., \& McMillan, S. J. (1999). Response variation in email surveys: An exploration. Journal of Advertising Research, 39, 45-54.

Shih, T. H., \& Fan, X. (2008). Comparing response rates from web and mail surveys: A meta-analysis. Field Methods, 20, 249-271.

Shin, B., El Sawy, O.A., Sheng, O.R.L., \& Higa, K. (2000). Telework: Existing research and future directions. Journal of Organizational Computing and Electronic Commerce, 10(2), 85-101.

Shoenfelt, E. L., Kottke, J. L., \& Stone, N. J. (2012). Master's and undergraduate industrial/organizational internships: Data-based recommendations for successful experiences.Teaching of Psychology, 39, 100-106.

Simons, L., Fehr, L., Blank, N., Connell, H., Georganas, D., Fernandez, D., \& Peterson, V. (2012). Lessons learned from experiential learning: What do students learn from a practicum/internship? International Journal of Teaching and Learning in Higher Education, 24, 325-334.

Simonson, M., Smaldino, S., \& Zvacek, S. (2015). Teaching and learning at a distance: Foundations of distance education (6th ed.). Charlotte, NC: Information Age Publishing.

Spooner, M., Flowers, C., Lambert, R., \& Algozzine, B. (2008). Is more really better? Examining perceived benefits of an extended student teaching experience. Clearing House: A Journal of Educational Strategies, Issues and Ideas, 81, 263270.

Sports Management Worldwide (SMWW). (2017, August). Virtual internship program. Retrieved from http://www.sportsmanagementworldwide.com/virtual-internshipprogram

Stake, R. E. (2013). Multiple case study analysis. Guilford Press.

Steffes, J. S. (2004). Creating powerful learning environments beyond the classroom. Change, 36(3), 46-50.

Stichman, A. J., \& Farkas, M. A. (2005). The pedagogical use of internships in criminal justice programs: A nation-wide study. Journal of Criminal Justice Education, 16(1), 145-165. doi:10.1080/1051125042000333523 
Stirling, A. A., Kerr, G., MacPherson, E., Banwell, J., Bandealy, A., \& Battaglia, A. (2017). Do postsecondary internships address the four learning modes of experiential learning theory? An exploration through document analysis. Canadian Journal of Higher Education, 47(1), 27-48.

Stogdill, R.M. (1974). Handbook of Leadership: A Survey of Theory and and Research. New York: Free Press.

Stogdill, R.M. \& Coons, A.E. (1957). Leader behavior: Its description and measurement. Columbus OH: Ohio State University, Bureau of Business Research.

Straus, S. E., Johnson, M. O., Marquez, C., \& Feldman, M. D. (2013). Characteristics of successful and failed mentoring relationships: a qualitative study across two academic health centers. Academic Medicine: Journal of the Association of American Medical Colleges, 88(1), 82.

Sweitzer, H.F., \& King, M.A. (2014). The successful internship: Personal, professional, and civic development in experiential learning (4th ed.). Belmont, CA: Brooks/Cole Cenage.

Taylor, S. M. (1988). Effects of college internships on individual participants. Journal of Applied Psychology, 73, 393-401.

Taylor, P. (1994). Leadership in education. Emergency Librarian, $21(3), 9$.

Templeton, W., Updyke, K. \& Bennet, R.B. (2012). Internships and the assessment of student Learning. Business Education \& Accreditation, 6 (2), 27-38.

The Viscardi Center. (2017, September). Are virtual internships on the horizon? Retrieved from https://www.viscardicenter.org/virtual-internships-html/.

Thiel, G. R., \& Hartley, N. T. (1997). Cooperative education: A natural synergy between Business and academia. SAM Advanced Management Journal, 62, 19-24.

Tourmen, C., Leroux, A., \& Beney, S. (2012). What is learned during the first moments of work? Work, 41, 5231-5234.

Tracy, D. (1990). The power pyramid: How to get power by giving it away. New York: Harper Collins.

Tracy, S. J. (2010). Qualitative quality: Eight "big-tent" criteria for excellent qualitative research. Qualitative Inquiry, 16(10), 837-851.

Transcribe. (2018). Transcribe. Retrieved from https://transcribe.wreally.com/ 
U.S. Mission Haiti. (2017, July 17). Virtual internship opportunity with the federal government. Retrieved from https://ht.usembassy.gov/virtual-internshipopportunity-federal-government/.

University of Virginia. (2017). Virtual internships. Retrieved from https://career.virginia. edu/internships/prepare/virtual-internships.

U.S. Journal of Academics. (2017, August). USjournal's un-paid virtual internship opportunity. Retrieved from http://www.usjournal.com/info/internship.php

Vaccaro, N., \& Lambie, G.W. (2007). Computer based counselor-in-training supervision: Ethical and practical implications for counselor educators and supervisors. Counselor Education \& Supervision, 47(1), 46-57.

Vasella, D. (2011). The path-goal theory of leadership. Thousand Oaks: Sage Publications.

Victor, L. (2013). Making a long-term impact on students through a place-based experiential approach to academics. Curriculum and Teaching Dialogue, 15(1\&2), 83-96.

Virtual Student Foreign Service (VSFS). (2017). Welcome to VSFS. Retrieved from https://vsfs.state.gov/about.

Vizcaya-Moreno, M. F., Pérez-Cañaveras, R. M., De Juan, J., \& Saarikoski, M. (2015). Clinical Learning Environment, Supervision and Nurse Teacher Evaluation Scale--Spanish Version [Database record]. Retrieved from PsycTESTS. doi: http://dx.doi.org /10.1037/t38492-000

Vroom, V. H. (1964). Work and motivation. San Francisco, CA: Jossey-Bass.

Walmsley, A., Thomas, R. \& Jameson, S. (2012). Internships in SMEs and career intentions. Journal of Education and Work, 25(2), 185-204.

WayUp. (2017, August). 2017 virtual internships. Retrieved from https://www.wayup .com/s/internships/virtual/\#.

Weible, R. (2009). Are universities reaping the available benefits internship programs offer?. Journal of Education for Business, 85(2), 59-63.

Weinert, C., Maier, C., Laumer, S., \& Weitzel, T. (2014, May). Does teleworking negatively influence IT professionals? An empirical analysis of IT personnel's telework-enabled stress. In Proceedings of the 52nd ACM Conference on Computers and People Research (pp. 139-147). ACM. 
Westera, W. (2011). On the changing nature of learning context: Anticipating the virtual extensions of the world. Journal of Educational Technology \& Society, 14(2), 201-n/a. Retrieved from http://search.proquest.com.ezproxylocal.library. nova.edu/docview /1287031392?accountid=6579.

Western Governors University (WGU). (2017, August). Virtual internships. Retrieved from https://indiana.wgu.edu/landing/internnet.

WhatsApp Inc. (2018). WhatsApp. Retrieved from https://www.whatsapp.com/.

Williams, R. (1990). The impact of field education on student development research findings. Journal of Cooperative Education, 27, 29-45.

Wendt, H., M. C. Euwema, \& I. J. Hetty van Emmerik. (2009). Leadership and team cohesiveness across cultures. The Leadership Quarterly, 20(3), 358-70.

Yin, R. K. (2009). Case study research: Design and methods (4 ed.). Los Angeles: Sage.

Yukl, G. A. 2006. Leadership in Organizations, 6/E. Upper Saddle River, NJ: Pearson Prentice Hall.

Yukl, G. A. 2013. Leadership in Organizations, 8/E. Upper Saddle River, NJ: Pearson Prentice Hall.

Zoom Video Communications, Inc. 2018. Zoom. Retrieved from https://zoom.us/. 
Appendix A

Case Study Protocol 


\section{Case Study Protocol}

\section{Purpose}

The purpose of the development of this protocol will structure and standardize the research project to increase the study's reliability.

\section{Overview of Project}

The problem this study will explore is the lack of research on how eSupervisory relationships with eInterns inform us about their learning contributions to society. A multiple case study analysis will be employed to collect information-rich data. The purpose of this study is to analyze to certain leadership methods that eSupervisors are utilizing with eInterns and the effectiveness of these methods on learning outcomes.

\section{Field Procedures}

1. Obtain proper permission to conduct the study from research site

2. Obtain approval from FIU Institutional Review Board

3. Identify sampling frame

4. Consult with key informants for purposeful sampling

5. Conduct pilot study

6. Gather, transcribe, and analyze results. Data collected was stored in an encrypted file on an encrypted computer that only the researcher had access to. Data will be saved until May 31, 2020.

7. Refine data collection plans necessary 
8. Send out participation letter via email to potential participants from sample. Email addresses from a database listed provided by the Program Manager at the research site.

9. Upon acceptance of participation, schedule interviews through Zoom webconferencing to allow for interviews to be recorded.

10. Gather, transcribe, and analyze results. Data collected was stored in an encrypted file on an encrypted computer that only the researcher had access to. Data will be saved until May 31, 2020.

11. Conduct steps $8-10$ per case group

12. Conduct cross-case analysis

13. Report results

\section{Case Study Report}

TBD based on the outcomes of the study. 
Appendix B

Interview Protocol for eSupervisors 


\section{Interview Protocol for eSupervisors}

Disclaimer

1. Provide participant with an overview of the project

a. Purpose of the study

b. Guiding research questions

c. Data collection procedures

2. Inform participant that pseudonyms will be used to store the data and report the results

3. Gain participant's consent to record the interview

Interview Schedule

Name:

Date:

Time:

Interview Questions

\section{Background}

1. Where are you from?

a. Where did you grow up?

b. What kind of schools did you go to?

2. Describe your network of support (family, friends, self, etc.)

3. Describe your work ethic

4. Which government agency do you work for?

5. How long have you been working there?

6. Describe your typical job responsibilities

7. Describe a typical day at work

8. What have your experiences been like while working there?

\section{Supervisory Style}

9. How do you motivate your eIntern?

10. How do you facilitate on-the-job training for your eIntern?

11. What are some strengths that you have noticed in your Intern?

a. How have you tried to help your eIntern enhance their performance? 
12. What are some weaknesses or issues that you have noticed with your eIntern?

a. How have you addressed these concerns?

13. How do you evaluate your eIntern's performance?

a. How often do you evaluate their performance?

\section{Learning Objectives}

14. What do you want your eIntern to learn from this experience?

15. What do you see your eIntern doing professionally after this experience?

\section{Overall Satisfaction}

16. Describe your overall experience with VSFS
a. Is this your first eIntern?
b. If no, how many have you previously had?

Follow-up questions will be asked based on participant responses

Demographic Information:

1. What is your sex?
a. Male
b. Female

2. What is your age?
a. $18-21$
b. $22-25$
c. $26-30$
d. $31-40$
e. $41-60$
f. $61+$

3. What is your race?

a. White, non-Hispanic

b. Black/African American, non-Hispanic

c. Hispanic

d. Asian/Pacific Islander

e. Other

4. What is the name of the collegiate institution you are attending? Please write full name only.
a. 
5. What is your degree classification status?
a. Graduate student
b. Undergraduate student
c. Recent graduate from graduate program
d. Recent graduate from undergraduate program
e. Other

6. If undergraduate, what is your student classification status?
a. Freshmen (0-30 credits)
b. Sophomore (30-60 credits)
c. Junior (60-90 credits)
d. Senior $(90+$ credits $)$
e. N/A 


\section{Appendix C}

Interview Protocol for Students (current eInterns) 


\section{Interview Protocol for Students (current eInterns)}

Disclaimer

1. Provide participant with an overview of the project

a. Purpose of the study

b. Guiding research questions

c. Data collection procedures

2. Inform participant that pseudonyms will be used to store the data and report the results

3. Gain participant's consent to record the interview

Interview Schedule

Name:

Date:

Time:

Interview Questions

\section{Background}

1. Where are you from?

a. Where did you grow up?

b. What kind of schools did you go to?

2. Describe your network of support (family, friends, self, etc.)

3. Describe your work ethic

4. Which government agency do you work for?

5. What motivated you to apply for work there?

6. Is this your first working experience?

a. If no, where have you worked in the past?

b. What have your experiences been like?

7. Describe your typical job responsibilities

8. What have your experiences been like while working there, so far?

\section{Supervisory Relationship}

9. Describe the working relationship between you and your immediate eSupervisor in the virtual internship program

a. What are the best parts of your relation between you and your eSupervisor? 
b. What do you not like about your relation between you and your eSupervisor?

c. What would you change about it?

10. What motivates you to do your job?

a. How does your eSupervisor motivate you to do your job?

11. How do you learn best?

a. How does your eSupervisor adapt to your learning needs?

12. What are some strengths that you have working in a virtual environment?

a. How has your eSupervisor tried to help you enhance your performance?

13. What are some weaknesses or issues that you have working in a virtual environment?

a. How has your eSupervisor addressed these concerns?

\section{Learning Objectives}

14. What are your goals coming into VSFS?

15. What have you learned so far?

16. What more do you want to learn from this experience?

17. What do you see yourself doing professionally after this experience?

a. How can you apply the skills you have learned from VSFS?

\section{Overall Satisfaction}

18. Describe your overall experience with VSFS

Follow-up questions will be asked based on participant responses

Demographic Information:

1. What is your sex?

a. Male

b. Female

2. What is your age?

a. $18-21$

b. $22-25$

c. $26-30$ 

d. $31-40$
e. $41-60$
f. $61+$

3. What is your race?
a. White, non-Hispanic
b. Black/African American, non-Hispanic
c. Hispanic
d. Asian/Pacific Islander
e. Other

4. What is the name of the collegiate institution you are attending? Please write full name only.

a.

5. What is your degree classification status?
a. Graduate student
b. Undergraduate student
c. Recent graduate from graduate program
d. Recent graduate from undergraduate program
e. Other

6. If undergraduate, what is your student classification status?
a. Freshmen (0-30 credits)
b. Sophomore (30-60 credits)
c. Junior (60-90 credits)
d. Senior $(90+$ credits $)$
e. N/A 


\section{Appendix D}

Interview Protocol for Graduates (past eInterns) 


\section{Interview Protocol for Graduates (past eInterns)}

\section{$\underline{\text { Disclaimer }}$}

1. Provide participant with an overview of the project
a. Purpose of the study
b. Guiding research questions
c. Data collection procedures

2. Inform participant that pseudonyms will be used to store the data and report the results

3. Gain participant's consent to record the interview

Interview Schedule

Name:

Date:

Time:

\section{Interview Questions}

\section{Background}

1. Where are you from?

a. Where did you grow up?

b. What kind of schools did you go to?

2. Describe your network of support (family, friends, self, etc.)

3. Describe your work ethic

4. Which government agency did you work for in VSFS?

5. What motivated you to apply for work there?

6. Was that your first working experience?

a. If no, where have you worked prior to that?

b. What have your experiences been like?

7. Describe the a typical work day with VSFS

8. What have your experiences been like while working there?

\section{Learning Outcomes}

9. What were your goals going into the VSFS program?

a. How were they achieved/Why weren't they achieved? 
10. What did you learn from that experience?

11. What more did you want to learn from that experience?

12. What was valuable to you about your experience at VSFS?

13. What have you done after that experience?

a. Where are you now?

b. Where do you want to go?

14. How are you applying the skills you have learned?

15. Describe your overall experience with VSFS

Follow-up questions will be asked based on participant responses

Demographic Information:

7. What is your sex?

a. Male

b. Female

8. What is your age?
a. $18-21$
b. $22-25$
c. $26-30$
d. $31-40$
e. $41-60$
f. $61+$

9. What is your race?

a. White, non-Hispanic

b. Black/African American, non-Hispanic

c. Hispanic

d. Asian/Pacific Islander

e. Other

10. What is the name of the collegiate institution you are attending? Please write full name only.

a.

11. What is your degree classification status?

a. Graduate student

b. Undergraduate student

c. Recent graduate from graduate program

d. Recent graduate from undergraduate program

e. Other 
12. If undergraduate, what is your student classification status?
a. Freshmen (0-30 credits)
b. Sophomore (30-60 credits)
c. Junior (60-90 credits)
d. Senior $(90+$ credits $)$
e. N/A 
Appendix E

Worksheets for Cross-Case Analysis 
Worksheet 2. The research questions or Themes of the multicase study and Factors that might be used in a more quantitative study.

\begin{tabular}{l|}
\hline Theme 1: \\
If more quantitative, Factors: \\
\hline Theme 2: \\
If more quantitative, Factors: \\
\hline Theme 3: \\
If more quantitative, Factors: \\
\hline Theme 4: \\
If more quantitative, Factors: \\
\hline Theme 5: \\
If more quantitative, Factors: \\
\hline Theme 6: \\
If more quantitative, Factors: \\
\hline
\end{tabular}


Case ID

Worksheet 3. Analyst's Notes while reading a case report

\begin{tabular}{|c|c|}
\hline Synopsis of case: & $\begin{array}{l}\text { Case Findings: } \\
\text { I. } \\
\text { II. } \\
\text { III. }\end{array}$ \\
\hline $\begin{array}{l}\text { Uniqueness of case situation } \\
\text { for program/phenomenon: }\end{array}$ & IV. \\
\hline $\begin{array}{l}\text { Relevance of case for cross-case Themes: } \\
\text { Theme } 1 \_ \text {Theme } 2 \\
\text { Theme } 4 \_ \text {Theme } 5 \text { Theme } 3 \\
\text { Theme } 6\end{array}$ & $\begin{array}{l}\text { Possible excerpts for cross-case report: } \\
\text { Page } \\
\text { Page } \\
\text { Page }\end{array}$ \\
\hline General influences (optional): & Situational Factors (optional) \\
\hline Commentary: & \\
\hline
\end{tabular}


Worksheet 4. Estimates of Ordinariness of the Situation of Each Case and Estimates of Manifestation of Multicase Themes in Each Case

$\mathbf{W}=$ highly unusual situation,$\quad \mathrm{u}=$ somewhat unusual situation, $\quad$ blank $=$ ordinary situation

$\mathbf{M}=$ high manifestation, $\mathrm{m}=$ some manifestation, blank = almost no manifestation

\begin{tabular}{|c|c|c|c|c|c|}
\hline & $\begin{array}{c}\text { Case } \\
\text { A }\end{array}$ & $\begin{array}{c}\text { Case } \\
\text { B }\end{array}$ & $\begin{array}{c}\text { Case } \\
\text { C }\end{array}$ & $\begin{array}{c}\text { Case } \\
\text { D }\end{array}$ & $\begin{array}{c}\text { Case } \\
\text { E }\end{array}$ \\
\hline \multicolumn{6}{|l|}{ Ordinariness of this Case's situation: } \\
\hline \multicolumn{6}{|l|}{ Original Multicase Themes } \\
\hline \multicolumn{6}{|l|}{ Theme 1} \\
\hline \multicolumn{6}{|l|}{ Theme 2} \\
\hline \multicolumn{6}{|l|}{ Theme 3} \\
\hline \multicolumn{6}{|l|}{ Theme 4} \\
\hline \multicolumn{6}{|l|}{ Theme 5} \\
\hline \multicolumn{6}{|l|}{ Theme 6} \\
\hline \multicolumn{6}{|l|}{ Added Multicase Themes } \\
\hline \multicolumn{6}{|l|}{$\begin{array}{ll}\text { Theme } 7 \\
\end{array}$} \\
\hline \multicolumn{6}{|l|}{ Theme 8} \\
\hline & & & & & \\
\hline & & & & & \\
\hline
\end{tabular}

High manifestation means that the Theme is prominent in this particular case study.

A highly unusual situation (far from ordinary) is one that is expected to challenge the generality of themes.

As indicated, the original themes can be augmented by additional themes even as late as the beginning of

the cross-case analysis. The paragraphs on each Theme should be attached to the matrix so that the basis

for estimates can be readily examined. 
Worksheet 5. A Map on which to make Assertions for the Final Report

\begin{tabular}{|r|r|r|r|r|r|r|l|l|}
\hline & \multicolumn{7}{|c|}{ Themes } \\
\hline Case A & 1 & 2 & 3 & 4 & 5 & 6 & 7 & 8 \\
\hline Finding I & & & & & & & & \\
\hline Finding II & & & & & & & & \\
\hline Finding III & & & & & & & & \\
\hline Finding IV & & & & & & & & \\
\hline Finding I & & & & & & & & \\
\hline Finding II & & & & & & & & \\
\hline Finding III & & & & & & & & \\
\hline & & & & & & & & \\
\hline Finding I & & & & & & & & \\
\hline Finding II & & & & & & & & \\
\hline Finding III & & & & & & & & \\
\hline Finding IV & & & & & & & & \\
\hline Finding V & & & & & & & & \\
\hline Case C Cases & & & & & & & & \\
\hline And so on for the remaining Case
\end{tabular}

A High mark means that the Theme is an important part of this particular case study and relevant to the theme. 
Worksheet 6. Multi-case Assertions for the Final Report

\begin{tabular}{|c|c|c|}
\hline$\#$ & Assertion & $\begin{array}{c}\text { Evidence in } \\
\text { Which Cases }\end{array}$ \\
\hline 1 & & \\
\hline 2 & & \\
\hline 3 & & \\
\hline 4 & & \\
\hline 5 & & \\
\hline 6 & & \\
\hline 7 & & \\
\hline & & \\
\hline & & \\
\hline
\end{tabular}




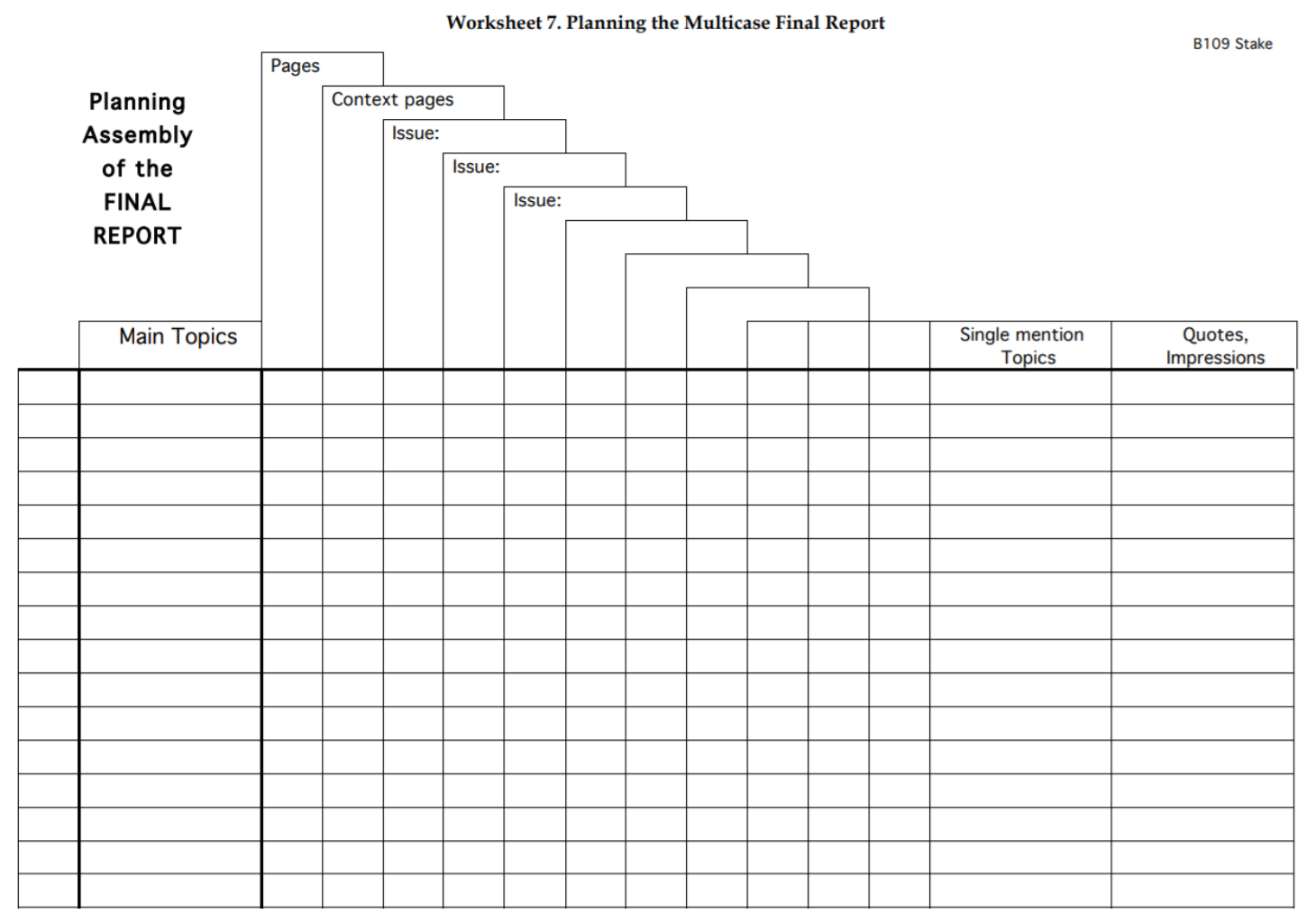

From Multiple Case Study Analysis, by Robert E. Stake. Copyright 2006 by The Guilford Press. Permission to photocopy these worksheets are granted to purchasers of this book for personal and professional use only (see copyright page for details).

Worksheets may be downloaded from http://www.guilford.com/p/stake. 


\section{Alignment of Research Components}

Each section on the interview protocols for the supervisors, eInterns, and graduates were designed to be similar in nature to triangulate the results of each case and gather general information about each participant. Each section will also be used to answer a specific research question that aligns with a component of PGTL. Please see chart and explanations below.

\section{Table 1}

\section{Alignment of Research Components}

\begin{tabular}{|c|c|c|}
\hline PGTL Components & Research Questions & Section of Interview Questions \\
\hline $\begin{array}{l}\text { PGTL focuses on the relationship between a leader and a subordinate or } \\
\text { follower (Yukl, 2013). The essence of PGTL is the meta proposition that } \\
\text { "leaders, to be effective, engage in behaviors that complement subordinates' } \\
\text { environments and abilities in a manner that compensates for deficiencies } \\
\text { and is instrumental to subordinate satisfaction and individual work unit } \\
\text { performance" (House, 1996, p. 324). }\end{array}$ & $\begin{array}{l}\text { How do eSupervisors describe their delivery of educational } \\
\text { contributions that promote learning in a virtual internship } \\
\text { environment? }\end{array}$ & $\begin{array}{l}\text { Supervisory Style } \\
\text { (ex: How do you motivate your eIntern? How do } \\
\text { you facilitate on-the-job training for your } \\
\text { eIntern? How have you tried to help your eIntern } \\
\text { enhance their performance? } \\
\text { How do you evaluate your eIntern's } \\
\text { performance?) }\end{array}$ \\
\hline $\begin{array}{l}\text { House and Mitchell (1974) advanced two general propositions in this } \\
\text { version: } \\
\begin{array}{ll}\text { 1) } \\
\text { Leader behavior is acceptable and satisfying to } \\
\text { subordinates to the extent that the subordinates see } \\
\text { such behavior as either an immediate source of } \\
\text { satisfaction or instrumental to future satisfaction } \\
\text { 2) } \\
\text { Leader behavior is motivational to the extent that (a) } \\
\text { such behavior makes satisfaction of subordinate's } \\
\text { needs contingent on effective performance and (b) } \\
\text { such behavior complements the environment of } \\
\text { subordinates by providing coaching, guidance, } \\
\text { support and rewards necessary for effective } \\
\text { performance (p. 84). }\end{array}\end{array}$ & $\begin{array}{l}\text { How do virtual interns interpret the educational contributions that } \\
\text { they receive from their supervisors into learning experiences? }\end{array}$ & $\begin{array}{l}\text { Supervisory Relationship } \\
\text { (ex. What are the best parts of your relation } \\
\text { between you and your eSupervisor? How does } \\
\text { your eSupervisor motivate you to do your job? } \\
\text { How does your eSupervisor adapt to your } \\
\text { learning needs? How has your eSupervisor tried } \\
\text { to help you enhance your performance? }\end{array}$ \\
\hline $\begin{array}{l}\text { House's (1971) theory proposes that a follower's motivations are based on } \\
\text { three assumptions: 1) If effort is given, the goal can be achieved; 2) If the } \\
\text { goal is achieved, there will be a reward; and 3) The reward is considered to } \\
\text { be valuable. }\end{array}$ & $\begin{array}{l}\text { How do virtual internships deliver educational contributions that add } \\
\text { value to a virtual intern's post-internship experience? }\end{array}$ & $\begin{array}{l}\text { Learning Outcomes } \\
\text { (ex: What did you learn from that experience? } \\
\text { What more did you want to learn from that } \\
\text { experience? What was valuable to you about your } \\
\text { experience at VSFS? How are you applying the } \\
\text { skills you have learned?) }\end{array}$ \\
\hline
\end{tabular}


Table 2

Major Theme: Supervisory Style

\begin{tabular}{cccccc}
\hline Initial Codes & S1 & Expanded Codes & Final Codes \\
\hline Motivation & Provides Work & $\begin{array}{c}\text { Sands-off; } \\
\text { Empowerment }\end{array}$ & $\begin{array}{c}\text { Malleability Work; Creative } \\
\text { Ownership }\end{array}$ & Ownership over projects & $\begin{array}{c}\text { Empowerment } \\
\text { Responsibility }\end{array}$ \\
\hline Training & $\begin{array}{c}\text { Guidelines and } \\
\text { Corrections }\end{array}$ & Provides Resources & $\begin{array}{c}\text { Provides Resources; Discussions; } \\
\text { Trial and Error }\end{array}$ & $\begin{array}{c}\text { Provides examples of } \\
\text { prior work }\end{array}$ & Resources \\
& Case-by-Case & Weekly & Monthly & Monthly & Regular \\
Evaluation & Efficiency & Become a self-starter & Support; Make mistakes & Become a critical thinker & Responsibility \\
\hline $\begin{array}{c}\text { Learning } \\
\text { Goals }\end{array}$ & & & & & \\
\hline
\end{tabular}


Table 3

Major Theme: Supervisory Relationship

\begin{tabular}{|c|c|c|c|c|c|c|c|c|c|}
\hline \multirow{2}{*}{$\begin{array}{l}\text { Initial } \\
\text { Codes }\end{array}$} & \multicolumn{8}{|c|}{ Expanded Codes } & \multirow[t]{2}{*}{ Final Codes } \\
\hline & Student 1A & Student 1B & Student 2A & $\begin{array}{c}\text { Student } \\
\text { 2B }\end{array}$ & Student 3A & Student 3B & $\begin{array}{c}\text { Student } \\
4 \mathrm{~A}\end{array}$ & $\begin{array}{c}\text { Student } \\
\text { 4B }\end{array}$ & \\
\hline $\begin{array}{l}\text { Working } \\
\text { Relationship }\end{array}$ & $\begin{array}{l}\text { Accessible; } \\
\text { Constant } \\
\text { Feedback }\end{array}$ & $\begin{array}{l}\text { Straight- } \\
\text { forward; } \\
\text { Minimal } \\
\text { dialogue }\end{array}$ & Great & $\begin{array}{l}\text { Easy- } \\
\text { going }\end{array}$ & $\begin{array}{l}\text { Approachable; } \\
\text { Communicative; } \\
\text { Caring }\end{array}$ & $\begin{array}{c}\text { Great; } \\
\text { Communicative }\end{array}$ & $\begin{array}{l}\text { Basic; } \\
\text { Minimal }\end{array}$ & $\begin{array}{l}\text { Little } \\
\text { contact; } \\
\text { no } \\
\text { guidance }\end{array}$ & Communication \\
\hline Motivation & Self & Self & Self & Self & Self & Self & Self & Self & Self-Initiated \\
\hline $\begin{array}{l}\text { Facilitation } \\
\text { of Learning }\end{array}$ & Unnecessary & None & Empowerment & Training & Trial and Error & Experimental & Self & Self & Self-Initiated \\
\hline
\end{tabular}


Table 4

Major Theme: Learning Outcomes

\begin{tabular}{|c|c|c|c|c|c|c|c|c|c|}
\hline \multirow{2}{*}{$\begin{array}{l}\text { Initial } \\
\text { Codes }\end{array}$} & \multicolumn{8}{|c|}{ Expanded Codes } & \multirow[t]{2}{*}{ Final Codes } \\
\hline & $\begin{array}{c}\text { Graduate } \\
\mathbf{1 A} \\
\end{array}$ & $\begin{array}{c}\text { Graduate } \\
\text { 1B } \\
\end{array}$ & $\begin{array}{c}\text { Graduate } \\
2 \mathrm{~A}\end{array}$ & Graduate 2B & $\begin{array}{c}\text { Graduate } \\
\mathbf{3 A} \\
\end{array}$ & $\begin{array}{c}\text { Graduate } \\
\text { 3B } \\
\end{array}$ & $\begin{array}{c}\text { Graduate } \\
\mathbf{4 A}\end{array}$ & Graduate 4B & \\
\hline $\begin{array}{l}\text { Knowledge } \\
\text { Gained }\end{array}$ & $\begin{array}{l}\text { Subject- } \\
\text { matter }\end{array}$ & $\begin{array}{l}\text { Subject- } \\
\text { matter }\end{array}$ & $\begin{array}{c}\text { Value of } \\
\text { Feeling } \\
\text { Appreciated }\end{array}$ & $\begin{array}{l}\text { Management of } \\
\text { Self; } \\
\text { Accountability }\end{array}$ & $\begin{array}{l}\text { How to } \\
\text { work } \\
\text { remotely }\end{array}$ & $\begin{array}{l}\text { Subject- } \\
\text { matter }\end{array}$ & $\begin{array}{l}\text { How to } \\
\text { work } \\
\text { remotely }\end{array}$ & $\begin{array}{c}\text { Time } \\
\text { Management }\end{array}$ & $\begin{array}{l}\text { Subject- } \\
\text { Matter }\end{array}$ \\
\hline $\begin{array}{c}\text { Valued } \\
\text { Experience }\end{array}$ & Flexibility & Opportunity & $\begin{array}{l}\text { Relationship } \\
\text { with } \\
\text { Supervisor }\end{array}$ & $\begin{array}{l}\text { Relationship } \\
\text { with Supervisor }\end{array}$ & $\begin{array}{l}\text { Impact of } \\
\text { work }\end{array}$ & Flexibility & $\begin{array}{l}\text { Self- } \\
\text { guided }\end{array}$ & $\begin{array}{l}\text { Flexibility; } \\
\text { Self- } \\
\text { regulation }\end{array}$ & $\begin{array}{c}\text { Self- } \\
\text { Management }\end{array}$ \\
\hline $\begin{array}{l}\text { Transfer of } \\
\text { Learning }\end{array}$ & $\begin{array}{c}\text { Time } \\
\text { Management }\end{array}$ & $\begin{array}{l}\text { Subject- } \\
\text { matter }\end{array}$ & $\begin{array}{l}\text { Good } \\
\text { working } \\
\text { relations }\end{array}$ & $\begin{array}{c}\text { Working } \\
\text { Independently; } \\
\text { Critical } \\
\text { Thinking }\end{array}$ & $\begin{array}{l}\text { Self-starter; } \\
\text { Transition } \\
\text { to graduate } \\
\text { school }\end{array}$ & $\begin{array}{c}\text { Future } \\
\text { endeavors }\end{array}$ & None & $\begin{array}{c}\text { Time } \\
\text { Management }\end{array}$ & Accomplished \\
\hline
\end{tabular}




\section{VITA}

\section{ELIZABETH ADADI}

Born, Brooklyn, New York

2006-2010

B.A., Psychology

Florida International University

Miami, Florida

2007-2008

Peer Advisor

Florida International University

Miami, Florida

2010-2012

M.A., Educational Administration

San Francisco State University

San Francisco, CA

2010-2018

Soldier

United States Army

United States of America

2014-2015

Academic Advisor

Florida International University

Miami, Florida

2015-2016

2015-2017

2014-2018

Graduate Teaching Assistant

Florida International University

Miami, Florida

Student Success Coach

Florida International University

Miami, Florida

Ed.D., Instructional Technology and Distance Education

Nova Southeastern University

Davie, Florida

2015-2018

Ph.D., Curriculum and Instruction

Florida International University

Miami, Florida 
Guardian Ad Litem

Florida Guardian ad Litem Program

Miami, Florida

\section{PUBLICATIONS AND PRESENTATIONS}

Adadi, E. (2017, July). Mobile Technologies and Motivation ...Influential Drivers of Learner Control? $15^{\text {th }}$ International Conference on Education and Information Systems, Technologies and Applications, Orlando, FL.

Adadi, E. (2017, March). Distance Education and the International Student: Perspectives in Higher Education. 61st Annual Meeting of the Comparative and International Education Society, Atlanta, GA.

Adadi, E. (2016, June). Adequate Support for Distance Learners: An Ongoing Challenge for Distance Education Programs. Symposium at South Florida Education Research Conference, Miami, FL.

Adadi, E., Azael, J., \& Thirunarayanan, M.O. (2016, April). Graduate Students Connecting with Undergraduate Students in an Online Course. Poster Session at $27^{\text {th }}$ International Conference on College Teaching and Learning, Jacksonville, FL.

Adadi, E. \& Saint-Louis, J. (2016, February). Career Planning Integrated into Academic Development: Important Aspects of a Student's Life. Paper Session at NACADA International Conference, Dubai, UAE.

Adadi, E., Troche, M., \& Gordon, A. (2016, February). Financial Readiness: A Key Factor in the Persistence of College Students. Paper Session at NACADA International Conference, Dubai, UAE

Adadi, E. (2015, October). MyMajorMatch: A Career Planning Tool. Best Practices Session at ELEARN - World Conference on E-Learning, Kona, Hawaii.

Adadi, E. (2015, October). Student Support Services in Distance Learning Environments...An Overlooked Component. Best Practices Session at E-LEARN - World Conference on E-Learning, Kona, Hawaii.

Adadi, E., \& Harris, S. (2015, June). Advising for the $21^{\text {st }}$ Century: How to Use Technology as a Tool to Achieve Academic Advising Learning Outcomes. Concurrent Session at NACADA International Conference, Melbourne, Australia. 